\title{
LUT
}

University

\section{Carbon-based quantum particles: an electroanalytical and biomedical perspective}

Nekoueian Khadijeh, Amiri Mandana, Sillanpää Mika, Marken Frank, Boukherroubd Rabah, Szunerits Sabine

This is a Author's accepted manuscript (AAM) version of a publication published by Royal Society of Chemistry

in Chemical Society Reviews

DOI: $10.1039 / C 8 C S 00445 E$

Copyright of the original publication: () Royal Society of Chemistry 2019

Please cite the publication as follows:

Nekoueian, K., Amiri, M., Sillanpää, M., Marken, F., Boukherroubd, R., Szunerits, S. (2019). Carbon-based quantum particles: an electroanalytical and biomedical perspective. Chemical Society Reviews, vol. 48, issue 15, pp. 4281-4316. DOI: 10.1039/C8CS00445E

This is a parallel published version of an original publication. This version can differ from the original published article. 


\title{
Carbon-Based Quantum Particles: An Electroanalytical and Biomedical Perspective
}

\author{
Khadijeh Nekoueian, ${ }^{1,2}$ Mandana Amiri, ${ }^{1 *}$ Mika Sillanpää, ${ }^{2}$ Frank Marken, ${ }^{3}$ Rabah \\ Boukherroub, ${ }^{4}$ Sabine Szunerits ${ }^{4 *}$ \\ ${ }^{1}$ Department of Chemistry, University of Mohaghegh Ardabili, Ardabil, Iran \\ ${ }^{2}$ Laboratory of Green Chemistry, School of Engineering Science, Lappeenranta University of \\ Technology, Sammonkatu 12, FI-50130 Mikkeli, Finland \\ ${ }^{3}$ Department of Chemistry, University of Bath, Bath BA2 7AY, United Kingdom \\ ${ }^{4}$ Univ. Lille, CNRS, Centrale Lille, ISEN, Univ. Valenciennes, UMR 8520-IEMN, F-59000 \\ Lille, France
}

To be submitted to Chemical Society Reviews

\begin{abstract}
Carbon-based quantum particles, especially spherical carbon quantum dots (CQDs) and nanosheets like graphene quantum dots (GQDs), are an emerging class of quantum dots with unique properties owing to their quantum confinement effect. Many reviews appeared recently in the literature highlightening their optical properties, structures, and applications. These papers cover a broad spectrum of carbon-based nanoparticles excluding a more detailed discussion about some important aspects related to the definition of carbon-based particles and the correlation of optical and electrochemical aspects in relation to sensing and biomedical applications. A large part of this review is devoted to these aspects. It aims in particular to act as a bridge between optical and electrochemical aspects of carbon-based quantum particles, both of which are associated with the electronic nature of carbon-based quantum particles. A special focus will be on their use in electroanalysis notably their benefits in redox and electrochemical analysis with emphases on their applicants as sensors. Electroanalysis is an easy and cost-effective means to give qualitatively and quantitatively information of a specific analyte in solution in a time scale of some minutes. The integration of carbon-based quantum particles into these detection schemes as well as their incorporation into composite nanomaterials has large improved detection limits with possibilities for their integration of for point-of-care devices up to personalized medicine. This review will emphasis on some of these aspects but will also include nanomedical aspects of carbon-based quantum particles, ultimately correlated for such developments.
\end{abstract}

\footnotetext{
${ }^{1}$ Correspondance should be send to: Mandand Amiri (mandanaamiri@uma.ac.ir), Frank Marken (f.marken@bath.ac.uk); Sabine Szunerits (sabine.szunerits@univ-lille.fr)
} 


\section{Content}

\section{Introduction}

2. Synthesis of carbon-based quantum particles

2.1. Top-down synthesis of carbon-based quantum particles

2.1.1. Arc discharge method

2.1.2. Laser ablation

2.1.3. Electrochemical synthesis

2.2. Bottom-up synthesis of carbon-based quantum particles

2.2.1. Chemical or thermal oxidation

2.2.2. Hydrothermal synthesis

2.2.3. Microwave synthesis

2.2.4. Supported routes

2.2.5. Large scale synthetic routs

3. Chemical and Physical Properties of carbon-based quantum particles

3.1. Chemical structure

3.2. Optical properties

3.3. Redox properties

3.4. Cell viability and toxicity

4. Surface Functionalization of carbon-based quantum particles

5. Applications

5. 1. Chemical and biological sensing

5.1.1. Photoluminescence sensors

5.1.2. Electrochemiluminescence sensors

5.1.3. Electrochemical sensors

5.2. Recent biomedical applications

5.2.1. Bioimaging

5.2.2. Drug delivery systems

5.2.3. Antibacterial and antiviral properties

5.2.4. Inhibition of protein aggregation

\section{Summery and Outlook}




\section{Introduction}

The interest in carbon-based quantum particles dates back to 2004, when fluorescent carbon quantum dots were accidentally formed during electrophoretic purification of single-walled carbon nanotubes. ${ }^{1}$ Carbon-based quantum particles are now part of a rapidly expanding field and many reviews appeared recently in the literature highlighting their optical properties, structures, and applications..$^{2-16}$ The term "quantum particle" is used to distinguish them from the much broader field of carbon nanoparticles (CNPs), including next to carbon blacks, a large variety of carbon nanocomposites. Carbon-based quantum particle are thus regarded as electronically single domain, which relates to the exciton size (typically $1-2 \mathrm{~nm}$ in carbon structures), and include carbon nanostructures of less than $10 \mathrm{~nm}$ in diameter, noted carbon quantum particles (CQDs) and graphene nanosheets of usually less than $10 \mathrm{~nm}$ in later size, co-called graphene quantum dots (GQDS). Due to their unique and tunable photoluminescence, exceptional physicochemical properties, remarkable conductivity, generally low cytotoxicity and excellent water dispensability, carbon-based quantum particle emerged as universal nanomaterials widely used in optical and electroanalytical applications as well as for nonmedical applications (Figure 1). Carbon-based quantum particles are as a consequence replacing semiconductor quantum dots, which, owing to their strong and tunable fluorescence emission, have been used for years in applications where stable and strong fluorescence is required. However, their high toxicity, caused by the presence of heavy metals, restricted their safe use in clinical settings. Replacing semiconductor quantum dots with carbon-based quantum particles overcomes this limitation by providing biocompatible particles at low cost production, exhibiting chemical inertness in addition to similar fluorescent properties. Where is the place of carbon-based quantum particle in the carbon nanomaterials family?

The carbon nanomaterials family is ranging from $\mathrm{sp}^{3}$-rich nanostructures such as nanodiamond (also known as diamond nanoparticles) to $\mathrm{sp}^{2}$-rich graphene nanosheets and graphene quantum dots (GQDs). Between these extremes, one finds a large variety of carbon nanostructures with varying $\mathrm{sp}^{2} / \mathrm{sp}^{3}$ ratio (Figure 1). Nanodiamonds are made up of a diamond core and are with a $\mathrm{sp}^{2}$ carbon shell and/or different surface functional groups. ${ }^{17}$ Carbon nanoparticle (CNPs) ${ }^{18,19}$ encompass a panel of nanomaterials including carbon black, amorphous carbon, carbon soot, carbon dots (C-dots) and core-shell nanocarbons, with no real scientific consensus on the term describing them. Carbon black, for example, is the generic name of carbon particles which are produced by incomplete combustion of gaseous or liquid hydrocarbons. ${ }^{20}$ Amorphous carbon, informally known as coal or carbon soot, is an allotrope of carbon without crystalline structure, consisting of a mixture of $\mathrm{sp}^{3}$ and $\mathrm{sp}^{2}$ carbon, which converts into graphite upon graphitization at high temperature. These particles are sometimes also referred to as carbon nandots with overall diameter of about $10 \mathrm{~nm}$, existing often as aggregates to give particles of $20-50 \mathrm{~nm}$ in diameter. ${ }^{21}$ 


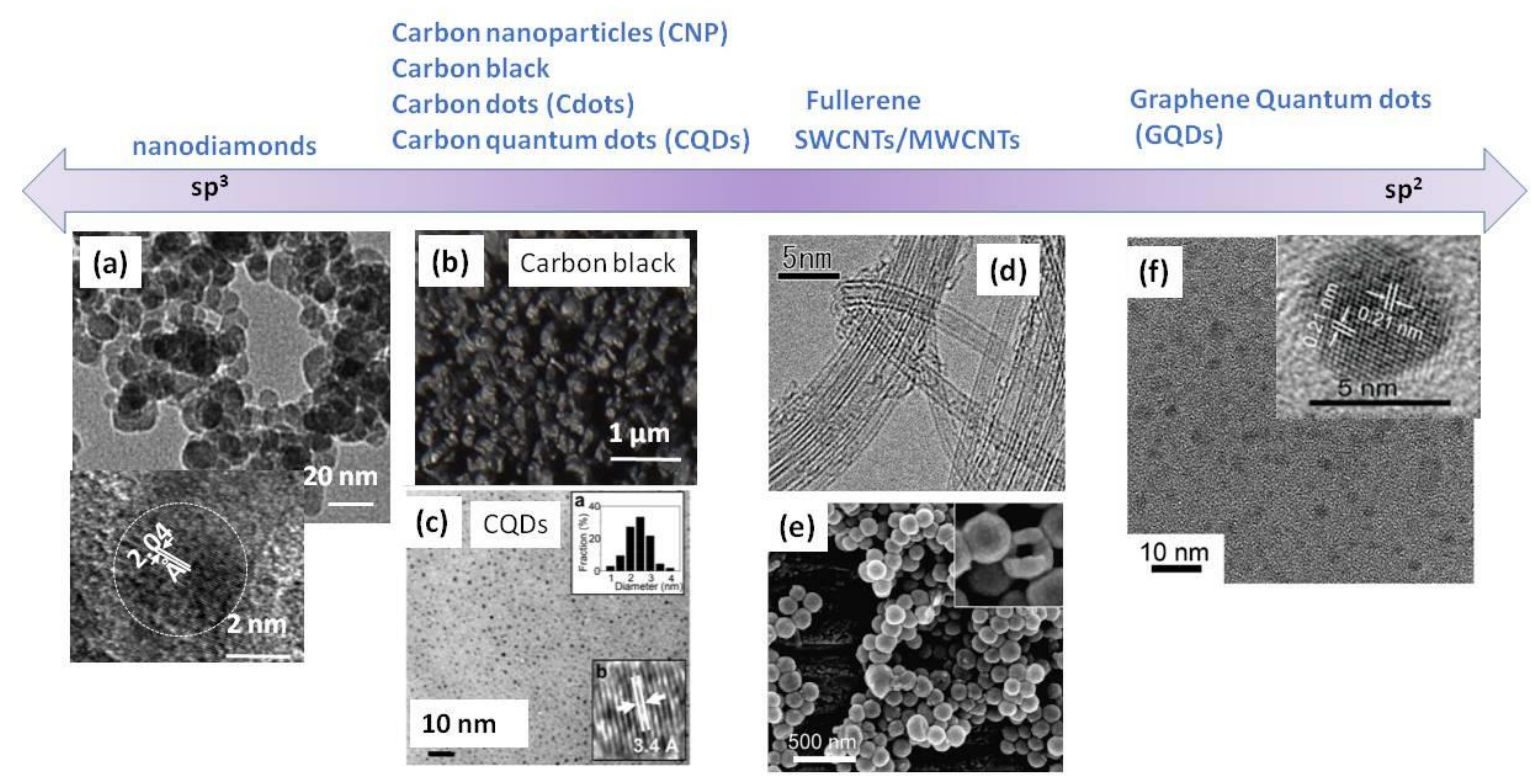

Figure 1: Carbon-based nanomaterials including carbon quantum particles: (a) TEM and HR-TEM images of carboxylated detonation nanodiamonds; (b) SEM image of carbon black, (c) SEM image of carbon quantum dots (CQDs); (d) TEM image of MWCNTs, (e) SEM image of $\mathrm{C}_{60}$ hollow spheres (reprint with permission from Ref. ${ }^{22}$ ); (f) TEM image (HRTEM inset) of graphene quantum dots (GQDs).

So what makes a carbon nanostructure a carbon quantum particle? Is there any real difference between carbon quantum dots, graphene quantum dots, carbon dots, carbon nanoparticles, etc. definitions widely used without any real consensus in the literature?

Carbon quantum particles are a class of zero-dimensional nanomaterials that exhibit discrete quantized energy levels and different densities of states and have an obvious crystal lattice, which marks its difference to carbon nanodots. ${ }^{7}$ In contrast to other carbon nanostructures, the properties of carbon quantum particles are largely determined by quantum mechanical effects and several parameters such as band gap, band edge position and density of states, being constant in bulk carbon, become dependent on the dimensions of the carbon nanomaterials (Figure 2). Shrinking CQDs in size changes the state density from a band structure to discrete energy levels. That is the reason why carbon quantum particles are largely known under the name carbon quantum dots (CQDs). As the size of the particles decreases to molecular or atomic size, the exciton (electron-hole pair) is further confined and the energy band gap structure becomes identical to that of a molecular electronic structure.

In other words, the photoluminescence properties vary sensitively with the size of the carbon quantum particles due to dependence of the HOMO-LUMO gap on the size, with increasing size resulting in a red-shifted emission. Optical quantum confinement effects occur when the size of the nanostructure approaches the Bohr excitation radius (e.g. 1-2 $\mathrm{nm}$ in carbon nanotubes), ${ }^{23}$ which correlates to carbon-based quantum particles of 2-10 $\mathrm{nm}$ in diameter. The electronic structure of carbon quantum particles can be probably best described using a modified Jablonski diagram as shown below in Figure 2c. Upon excitation, non-radiative 
processes dissipate energy from $S_{1}$ and the emitted photon has less energy than the initial excitation energy, resulting in a red-shifted emission compared to the excitation. The role of the defect state is as upon excitation, non-radiative processes occur which transfer energy to the defect states with some amount of energy loss. Once there, the emitted photon from the defect state is substantially red-shifted compared to the excitation energy. One direct consequence of the quantum effect is the photoluminescence character of CQDs, which is size dependent, whereas carbon nanoparticles necessitate modification of surface properties through surface passivation techniques to stabilize the optical properties and to allow tuning of photoluminescence features. ${ }^{7}$ The energy gap in the CQDs is divided into several levels with fluorescence occurring through excitation and emission within the core of the quantum dots.

Indeed, next to quantum effects, features related to surface composition become dominant upon decreasing the size of the nanostructures. More atoms are taking the role of edge and corner sites, and the effect of functional groups on the physico-chemical properties of the carbon-based are becoming more pronounced. ${ }^{24-27}$ That the emission peak position is often related to the excitation wavelength $\left(\lambda_{\text {ex }}\right)$ is currently believed to be a result of optical selection of nanoparticles of different sizes (quantum effect) and/or from surface chemistry, and different emissive traps on the quantum particle surface issues. This indicates that most of the CQDs are currently non-uniform particles. However, more and more cases are emerged with an $\lambda_{\text {ex }}$ independent emission position due to the ability to control the size uniformity and surface chemistry. ${ }^{28}$ 
(a)

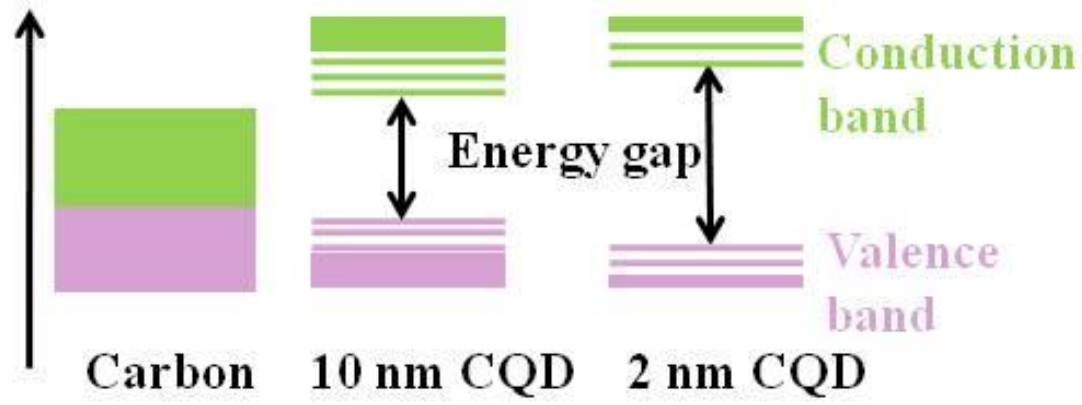

(b)

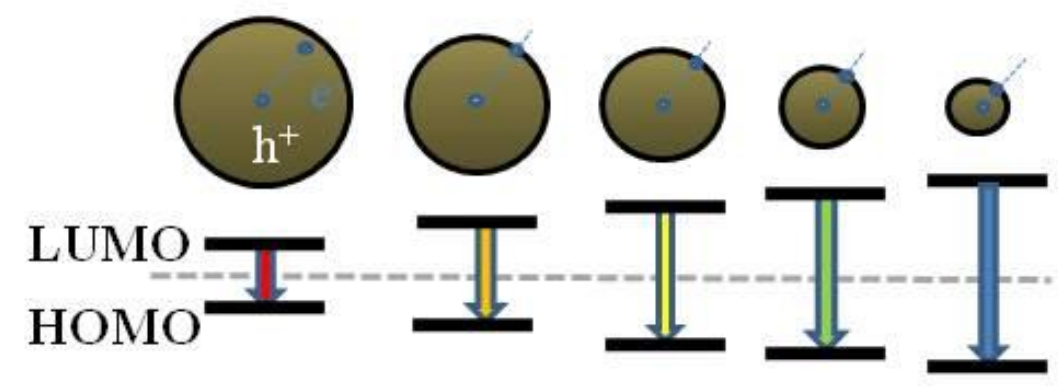

(c)

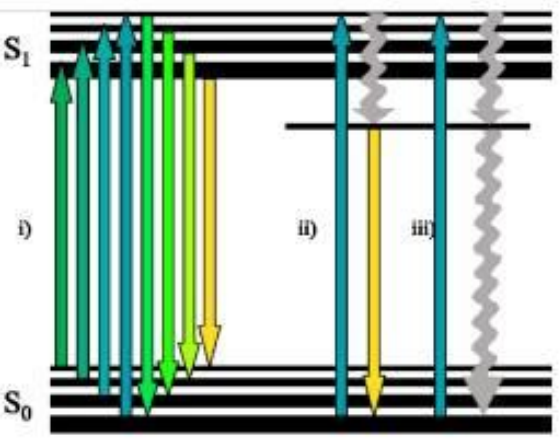

(d)

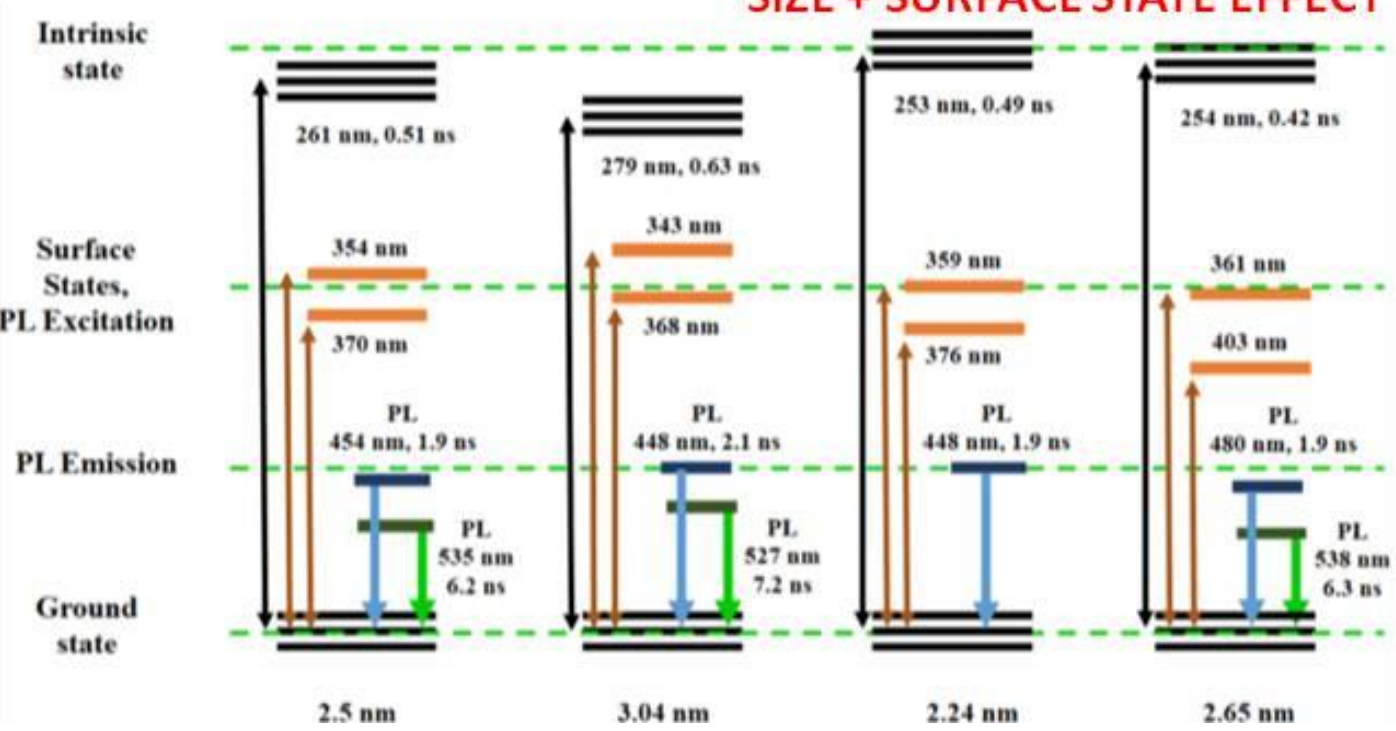

Figure 2: Quantum confinement effects in carbon-based particles: (a) Electronic structures of bulk carbon, $10 \mathrm{~nm}$ and $20 \mathrm{~nm}$ CQDs; (b) Effect of particle size on HOMOLUMO gap; (c) Modified Jablonski diagram to illustrate the electronic structure of CQDs; (d) Proposed energy levels of GQDs depending on size and surface function (reprint with permission of Ref. ${ }^{29}$ ). 
The distinction between carbon (CQDs) and graphene quantum dots (GQDs) is limited to mainly morphology. GQDs are crystalline zero-dimensional disks of graphene in the size of 2-20 nm, while CQDs are spherical particles comprising an amorphous to monocrystalline core with predominantly graphitic ( $\mathrm{sp}^{2}$ carbon) shell. ${ }^{7}$ While there is a lot of confusion in the literature, both materials are being part of the family of carbon quantum particles (Figure 1), consisting of $\mathrm{C}, \mathrm{H}, \mathrm{N}$ and $\mathrm{O}$ present in the form of various groups providing water solubility and surface functionalization. CQDs as well as GQDs often contain a considerable amount of carboxyl groups, which imparts the particles with excellent water dispersibility and suitable chemically reactive groups for further functionalization and surface passivation with various organic, inorganic and biological materials. These carbon-based quantum particles can also easily functionalized with heteroatoms or hybridized with different substances including metal oxides, biomolecules or conducting polymers to tailor their structural and physicochemical properties. ${ }^{30}$ In the early time of carbon-based quantum particles, enormous effort has been devoted to developing methods for their synthesis and surface modification. As the preparation method is fundamental to the resulting physic-chemical properties, a more detailed discussion is given in the next chapter.

\section{Synthesis of carbon-based quantum particles}

The diversity of carbon-based quantum particles, is linked to the large amount of synthetic methods available (Figure 3). A review by Shen et al. ${ }^{31}$ captures much of the current progress and the excitement, while the synthetic methods for the formation of GQDs have been reviewed recently in Ref. ${ }^{32}$ Here we give a global overview of methods applicable to the formation of carbon-based quantum particles without differentiation between CQDs and GQDs.
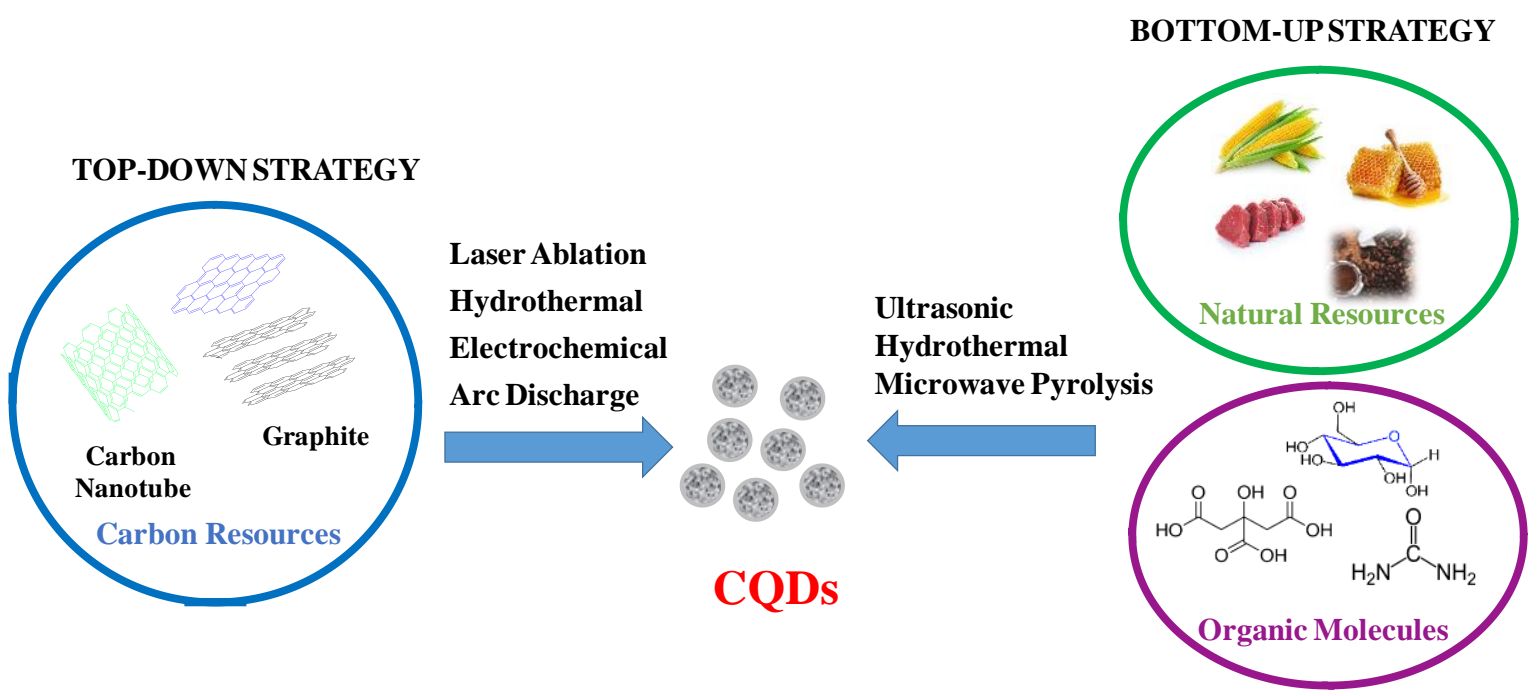

Figure 3: Synthetic approaches for carbon-based nanomaterials.

Independent of the carbon nanostructure aimed at, the synthetic approaches are generally classified into two main categories: top-down and bottom up. The top-down methods are based on breaking down larger carbon structures such as graphite powder, carbon rods, carbon 
nanotubes, carbon black and even candle soot, ${ }^{33}$ by methods such as laser ablation, ${ }^{34}$ hydrothermal, ${ }^{35,}{ }^{36}$ electrochemical oxidation, ${ }^{37,} 38$ or arc discharge. ${ }^{39}$ The advantage of the top-down approaches for the preparation of CQDs is that abundant raw materials can be used, allowing large-scale production and simple operation. The formed particles feature a high crystalline nature and excellent aqueous dispersibility. These carbon nanoparticles possess in general perfect $\mathrm{sp}^{2}$ carbon structure and often lack efficient gap to give fluorescence. The size and surface chemistry is thus modulated using oxidizing agents such as concentrated acids $\left(\mathrm{HNO}_{3}, \mathrm{H}_{2} \mathrm{SO}_{4} / \mathrm{HNO}_{3}\right.$ mixture), where bulk carbon materials are cut into small pieces, while the surface is modified with oxygen containing groups. This two-step route has become well accepted for the formation of GQDs based on the conversion of graphite into graphene oxide (GO) sheets using the Hummers method and the second step consists of cutting the GO into GQDs with various methods.

While the top-down strategy is based on breaking bulk carbon materials into small pieces by physical or chemical methods, the bottom-up approach uses selected molecular precursors, which upon dehydration and carbonization processes forms CQDs. The precursors possess generally $-\mathrm{OH},-\mathrm{COOH},-\mathrm{C}=\mathrm{O}$ and $\mathrm{NH}_{2}$ groups which can be dehydrated at elevated temperatures. There are countless of approaches to perform the dehydration and carbonization processes including hydrothermal, ${ }^{40}$ microwave-hydrothermal, ${ }^{41}$ plasma-hydrothermal approaches $^{42}$ etc. These methods offer exciting opportunities to control the molecular size and shape and fine tune the physico-chemical properties of CQDs as well as using green onestep synthetic approaches

In general, the bottom up approaches for the synthesis of CQDs are currently facing three issues: aggregation during carbonization, difficulties related to size control and achieving uniform properties as well as achieving suitable surface functions critical for solubility and selected applications. Post-treatments such as filtration, centrifugation, dialysis, gel electrophoresis, column chromatography are mostly required. Confined pyrolysis and electrochemical approaches are on the other hand well suited to overcome aggregation issues.

\subsection{Top-down synthesis of carbon-based quantum particles}

\subsubsection{Arc discharge method}

The arc discharge method has been widely applied for the preparation of carbon nanomaterials. ${ }^{43}$ Arc discharge is the electrical breakdown of a gas to generate a plasma. The chamber consists of two electrodes: one being the anode and filled with powdered carbon precursors, the other being the cathode usually a pure graphite rod (Figure 4A). After filling the chamber with a gas or a liquid and applying a power supply (AC or DC), the electrodes are brought into contact to generate an arc. During arc discharge, a large amount of heat is released resulting in the sublimation of the carbon precursors filled in the anode, and their drift towards the cathode where they are deposited in form of nanostructures. After oxidation of the crude materials (soot) in nitric acid, alkaline extraction and purification via gel electrophoresis, hydrophilic fluorescent CQDs are formed. ${ }^{33}$ The main drawback of this 
approach is the co-existence of a mixture of differently sized carbon nanostructures, often difficult to separate.

\subsubsection{Laser ablation}

A fast way to synthesize CQDs is based on laser ablation of carbon-based materials. The potential of large scale CQDs synthesis using laser ablation is nevertheless limited as the zone of target erosion is narrow and the synthetic yields are low. There are, however, several interesting reports using laser ablation for the synthesis of CQDs. Surface passivation can be achieved by further refluxing in $\mathrm{HNO}_{3}$ for $12 \mathrm{~h}$ and upon post-modification with polyethylene glycol (PEG) groups. Laser ablation of activated carbon (4\% ash) in a water/ethanol solution (5:2) was shown by Yogesh et al. to from CQDs of $4 \mathrm{~nm}$ in size. ${ }^{44}$ A one step process based on laser irradiation of a graphite target using the second harmonic beam at $532 \mathrm{~nm}$ of the $\mathrm{Nd}$ :YAG in $\mathrm{H}_{2} \mathrm{O}$ /ethanol or diethylenetriaminepentaacetic acid solution for the formation of 3 $\mathrm{nm}$ sized CQDs was reported by Tarasenka. ${ }^{45}$ The synthesized particles exhibited strong photoluminescence in the visible region. The laser ablation of a solid carbon material in liquid environment with laser pulses of 1064, 532 and $355 \mathrm{~nm}$ under different irradiation times was lately investigated. ${ }^{34}$ Wide CQDs size distribution was observed for the $1064 \mathrm{~nm}$ laser due to the deeper penetration of the laser light into the dielectric during the ablation process, being thus less adapted for the formation of size controlled CQDs.

\subsubsection{Electrochemical synthesis}

Electrochemical etching of different carbon-based electrodes is a viable low cost alternative for the formation of CQDs (Figure 4B). In this method, carbon electrodes such graphite rod electrode is electrochemically cut down into CQDs and GQDs. One of the first electrochemical synthesis of CQDs is that of Zhou et $a l^{46}$ who formed CQDs during electrochemical cycling of MWCNTs in the presence of tetra- $n$-butylammonium ions. Oxidation of graphitic electrodes at $+3.0 \mathrm{~V}$ was proposed by Zhao et al. ${ }^{28}$ to produce CQDs with emission wavelengths at $445 \mathrm{~nm}$ and $510 \mathrm{~nm}$. Independently, the electrochemical preparation of carbon nanocrystals was achieved by cycling between -3.0 and $+3.0 \mathrm{~V}$ at 0.1 $\mathrm{Vs}^{-1}$ of a carbon rod working electrode immersed in phosphate buffer solution (PBS). ${ }^{39}$ Alkali-assisted electrochemical etching allowed the preparation of CQDs with controlled size. ${ }^{47}$ Using graphite rods as anode and cathode and $\mathrm{NaOH} / \mathrm{EtOH}$ as electrolyte, CQDs were formed upon the application of a current in the $10-200 \mathrm{~mA} \mathrm{~cm}{ }^{-2}$ range. This work also underlines that an alkaline environment is one of the key factors for the synthesis of CQDs by electrochemical oxidation, as under acidic conditions no particles were formed.

Electrochemical synthesis of photo-luminescent CQDs from glycine under alkaline conditions has been recently proposed by Wang et al. ${ }^{38}$ Application of $+10 \mathrm{~V}$ between two Pt electrodes resulted in the oxidation of glycine and led to the formation of ammonium ions that further reacted with un-oxidized glycine through an amidation reaction. The as formed ions underwent electro-polymerization, carbonization and passivation to form highly fluorescent CQDs with an average size of $2.4 \pm 0.4 \mathrm{~nm}$ and crystalline nature. 
(A)

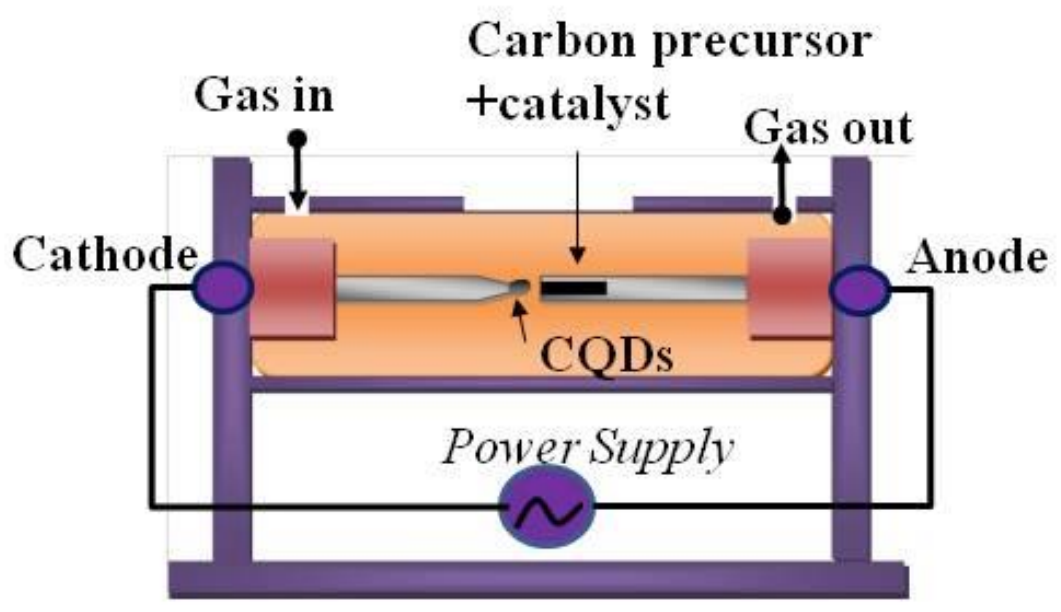

(B)

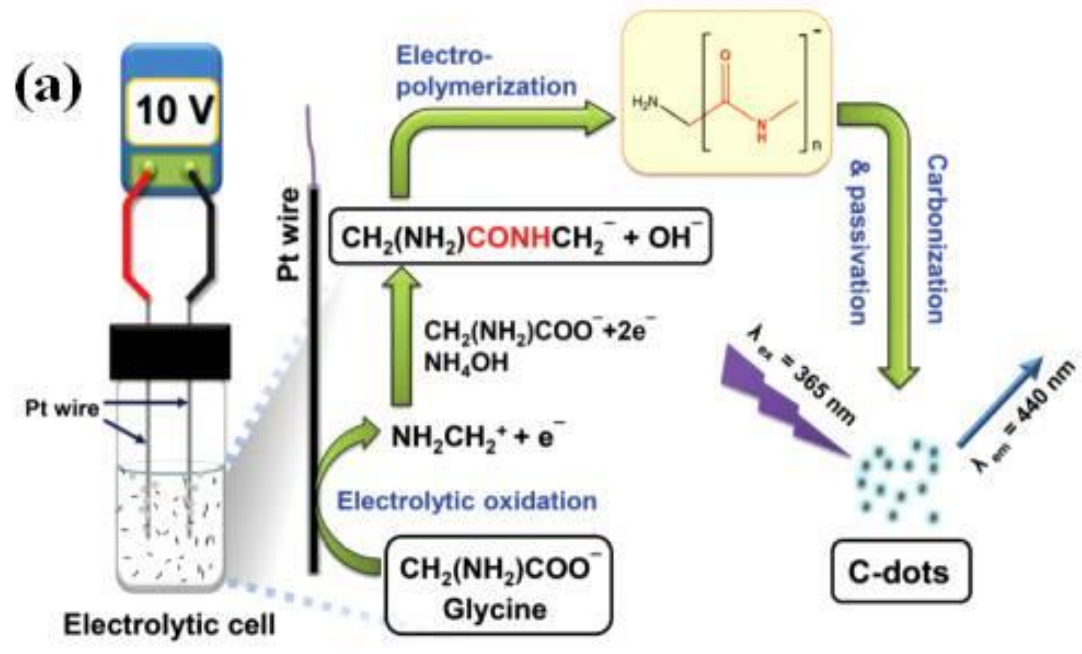

(b)

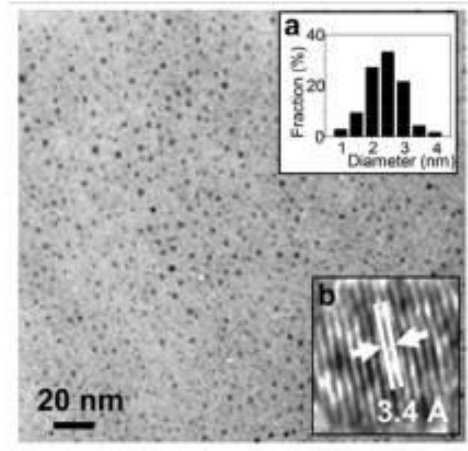

(c)

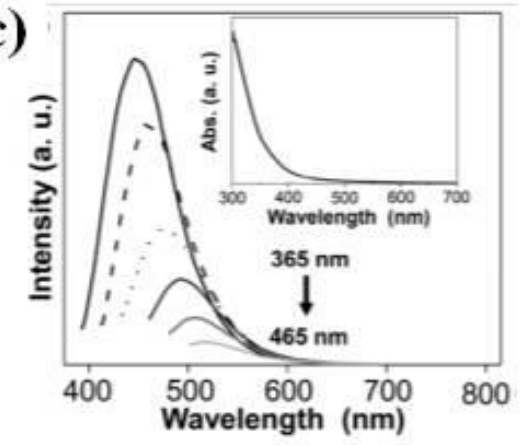

Figure 4: "Top-down" approaches for the synthesis of carbon-based quantum particles: (A) Schematic illustration of an arc discharge set up (reprint with permission from Ref. ${ }^{43}$ ); (B) (a) Preparation of CQDs from glycine through electro-oxidation, electro-polymerization, carbonization and passivation, (b) TEM image, (c) UV/Vis and photoluminescence spectra (reprint with permission from Ref. ${ }^{38}$ ). 


\subsection{Bottom-up synthesis of carbon-based quantum particles}

\subsubsection{Chemical or thermal oxidation}

For many years, the formation of carbon-based quantum particles has been achieved by pyrolysis or incomplete combustion of hydrocarbons. Polymer precursors were mostly employed as the fuel for pyrolysis should be thermally stable and able to yield useful carbon residues following high temperature processing. The carbonization process occurs through a number of different flame methods. Incomplete combustion of hydrocarbons leads to the formation of condensed carbonaceous particles (soot) in the nanometer diameter range, called carbon blacks. Inspired by this approach, it was found that soot derived from the combustion of candles or natural gas burners represents an excellent source for CQDs. In 2007, Liu et al. collected soot by placing a piece of aluminum foil atop a burning candle, then mixing with oxidizing agents and refluxed for $12 \mathrm{~h} .{ }^{48}$ CQDs smaller than $2 \mathrm{~nm}$ were collected by centrifugation or dialysis\} Chemical oxidation has several advantages including low cost, high yields of CQDs (>10\%) with large-scale production possibility. However, concentrated acids and bases along with long time treatments are needed for their preparation, resulting in fluorescent particles of often low quantum yields and poor size distribution. Dong et al. have developed a facile method to extract oxidized CQDs directly from activated carbon by chemical oxidation. ${ }^{49}$ Their formation is achieved by refluxing activated carbon in concentrated nitric acid for $24 \mathrm{~h}$, followed by neutralization with $\mathrm{NaOH}$ and dialysis against double distilled water. ${ }^{56}$ The synthesis of water soluble and photoluminescent carbon nanostructures from almond husk, a bio-waste was recently reported by Tripathy et al. ${ }^{50}$

\subsubsection{Hydrothermal synthesis}

Hydrothermal carbonization is a low cost, environmentally friendly and nontoxic route to produce carbon based materials from various precursors. This method consists of sealing an organic precursor within a closed system and heating at high temperature under reduced pressure. The interest of this approach lies in the good control of the nucleation processes in an environmentally friendly manner without the use of strong acids or bases. Precursors such as chitosan, ${ }^{51}$ carbohydrates,${ }^{52}$ and a large variety of green precursors, ${ }^{6}$ have been explored and the list is countless. 3-aminoproyltrimethoxy silane was lately considered as organic precursor to synthesize organosilane-functionalized fluorescent CQDs at $300^{\circ} \mathrm{C}$ in $2 \mathrm{~h}$ with a quantum yield of $42.6 \%$ without the introduction of an additional passivation agent. ${ }^{53}$ Among the disadvantages of this method is the possible formation of a carbon soot during the synthesis, which can reduce the yield. Sadhanala et al. ${ }^{54}$ reported the synthesis of CQDs of 2 and $7 \mathrm{~nm}$ in size using sucrose as a precursor without additional surface passivating agents or acids and bases. A one-pot hydrothermal synthesis of CQDs with efficient up and down converted PL was recently proposed by $\mathrm{Li}$ et al. (Figure 5A). ${ }^{55}$ This method allows in addition the formation of hydrophilic as well as hydrophobic particles as exemplified by Bhunia et al. ${ }^{56}$ 
(A)

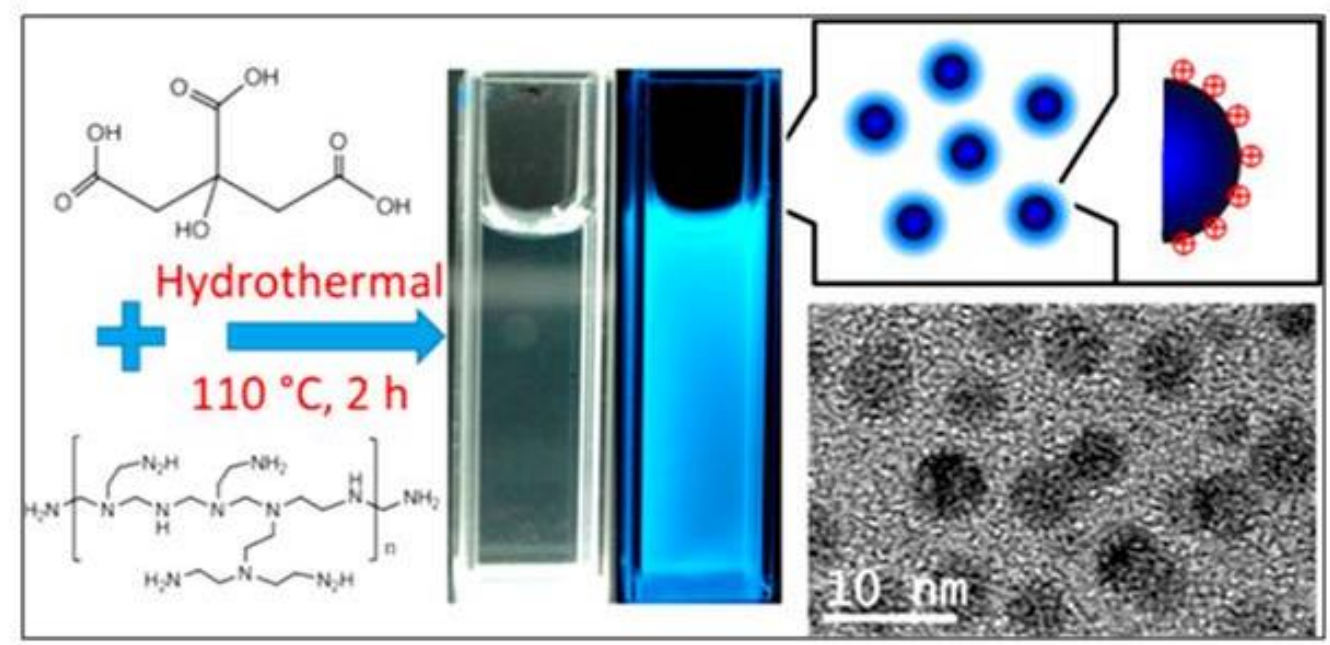

(B)

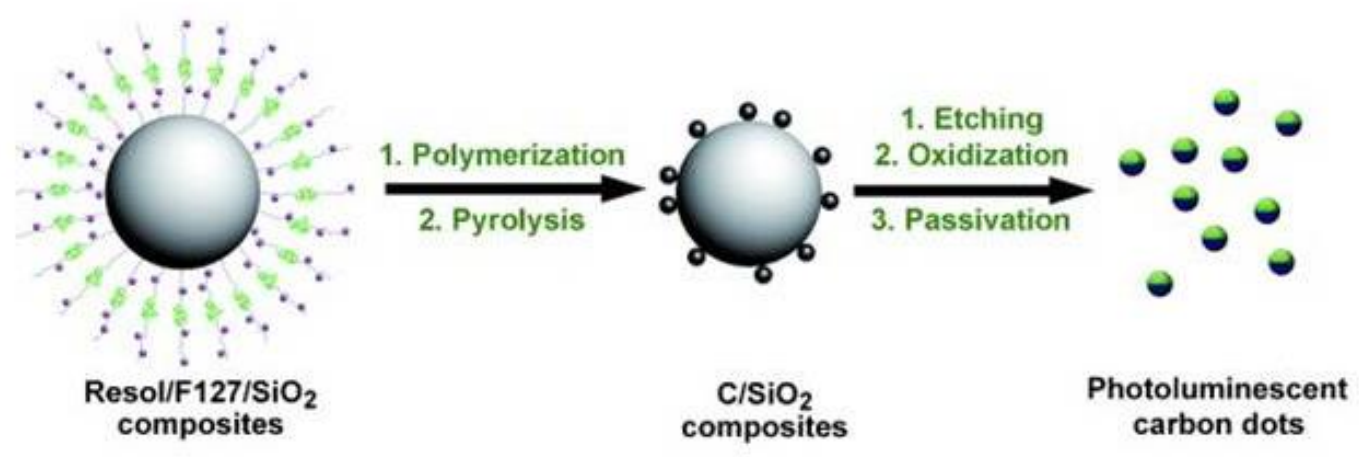

Figure 5: "Bottom-up" approaches for the synthesis of carbon-based quantum particles: particles. (A) Hydrothermal synthesis of CQDs using citrate and PEI precursors along with TEM image of the formed particles (reprint with permission from Ref. ${ }^{55}$ ); (B) Supported synthesis of CQDs using modified silica spheres as a carrier and resol as a carbon source (reprint with permission form Ref. ${ }^{57}$ ).

\subsubsection{Microwave synthesis}

Microwave technology, that can achieve high solution temperatures in some minutes, has become a very important process in synthetic chemistry while being a simple and a cheap approach for the fast synthesis of CQDs. Using sucrose as the carbon source and diethylene glycol as reaction medium resulted in the formation of green luminescent CQDs within only one minute under microwave irradiation. ${ }^{58}$ Liu et al. developed microwave-mediated pyrolysis of citric acid with various amine molecules to synthesize highly fluorescent CQDs. ${ }^{59}$ The amine molecules, especially primary amines, play a dual function as N-doping precursor and surface passivating agents. Synthesis of CQDs using poly(ethylene glycol (PEG) as precursor under oxygen atmosphere resulted in particles with remarkable photoluminescent properties. ${ }^{60}$ To decrease the synthesis time of CQDs and obtain different sizes and properties by varying irradiation power and duration, microwave synthesis is the best option. Yang et al. 
proposed a green and rapid microwave-assisted solid-phase synthesis (solvent-free) approach for the formation of highly fluorescence CQDs in $4 \mathrm{~min}$. The particles emit in the blue with a quantum yield of up to $63.2 \%$ in water solution and display yellow fluorescence in the solid state. ${ }^{61}$ Zhu et al. described a facile and economic microwave pyrolysis approach to synthesis fluorescent CQDs with electrochemiluminescence (ECL) properties. ${ }^{62}$

\subsubsection{Supported routes}

Discrete CQDs with uniform size can be prepared by confined pyrolysis of an organic precursor in nanoreactors. It is based on the adsorption of the organic precursor into a porous nanoreactor via capillary forces, followed by pyrolysis of the confined precursor into carbonaceous matter, and release by removing the nanoreactor. Porous silica is the most widely used nanoreactor, ${ }^{57}$ but polymer core-shell nanoparticles can also be efficiently used. One such a route was employed by Liu who used surfactant-modified silica spheres as supports (Figure 5B) ${ }^{57}$ Carbon precursor resols were introduced to the $\mathrm{SiO}_{2} / \mathrm{F} 127$ modified particles. The F127 surfactant phase is key as it is served as an anchor for the adsorption of resols through hydrogen bonding so that polymerization took place on the shell of the $\mathrm{SiO}_{2}$ spheres rather than in the solution. Heating to $900^{\circ} \mathrm{C}$ in $\arg$ on for $2 \mathrm{~h}$ led to $\mathrm{CQD} / \mathrm{SiO}_{2}$ composites where the silica sphere was removed through dissolution in $\mathrm{NaOH}$.

\subsubsection{Large scale synthetic routs}

Considering the great potential of CQDs for numerous applications, it is extremely necessary to consider large-scale synthetic routs. ${ }^{11}$ Most of the current methods and in particular hydrothermal synthesis due to its simplicity, low-coast, and high efficiency can be used to produce CQDs in the $\mathrm{mg}$ range regardless of the raw material used; however only a few works allow mass production, such as the carbonization of Chinese ink, where $120 \mathrm{~g}$ of CQDs were obtained. ${ }^{63}$ A simple one-pot microwave-assisted approach has been established by Ding and coworkers for the large-scale preparation of CQDs with excellent water solubility and photoluminescence. ${ }^{64}$ Using hydroquinone and ethylenediamine (EDA) for EDAcatalyzed decomposition of hydrogen peroxide at room temperature, the released heat from this highly exothermic reaction system was used to promote the formation of CQDs. ${ }^{65}$

\section{Chemical and Physical Properties of carbon-based quantum particles}

\subsection{Chemical structure}

The properties of carbon-based quantum particles depend strongly on their preparation rout. As a result, the chemical structures of CQDs show a large variability and characterization is of fundamental importance to get a better understanding of the formed carbon-based quantum particle. For example, GQDs, which can be composed of single or multiple graphene layers with chemical groups on the edges are generally anisotropic, with later dimensions larger than their thickness. Taking the example of GQDs, synthesized using pristine few-layer graphene flakes as a precursor, ${ }^{66}$ a narrow size distribution of 3-8 $\mathrm{nm}$ and tiny sheet-shaped morphology is observed using transmission electron microscopy (TEM) imaging (Figure 6A). Owing to the existence of a carbon core, GQDs process certain crystallinity, with, in case of 
GQDs formed from graphene flakes, a lattice spacing of $0.24 \mathrm{~nm}$ comparable to the (100) facet of graphite and a honeycomb lattice with zigzag edges of a $7 \mathrm{~nm}$ GQDs.

This contrasts to the spherical CQDs formed using ribonuclease $\mathrm{A}$ as a biomolecular templating agent under microwave irradiation, showing a typical interlayer distance of 0.34 $\mathrm{nm}$, corresponding to the (002) facet of graphite. ${ }^{67}$

\section{(A)}

\section{CQDs}

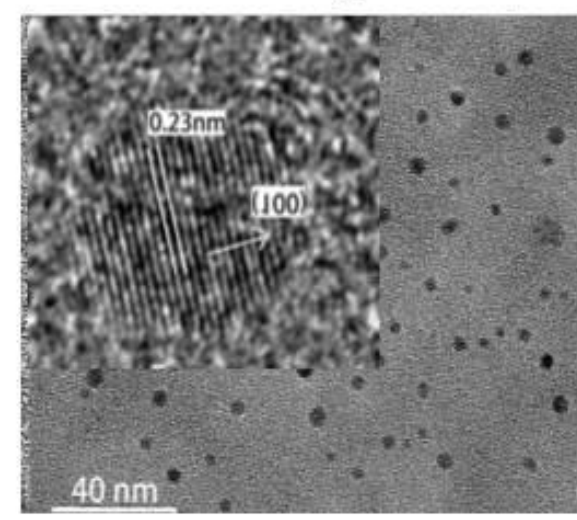

GQDs

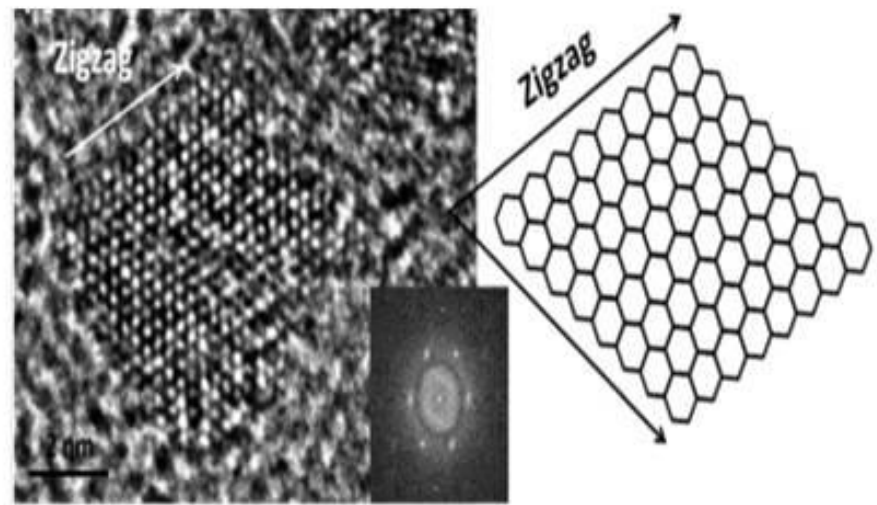

(B)

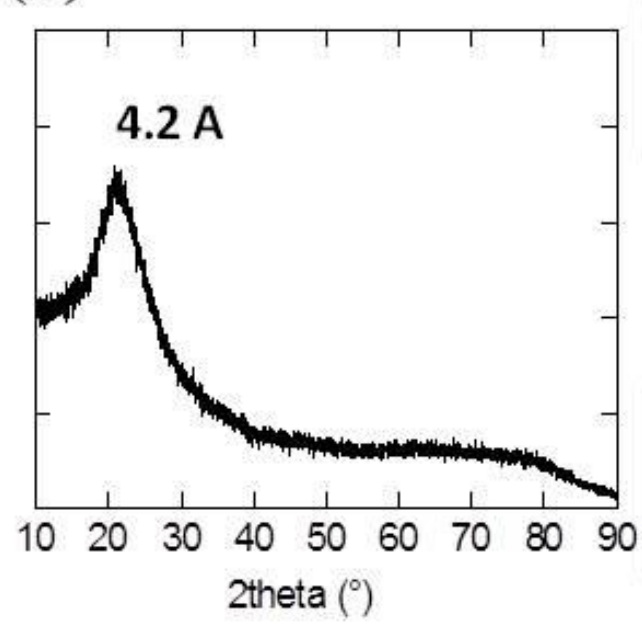

(a)

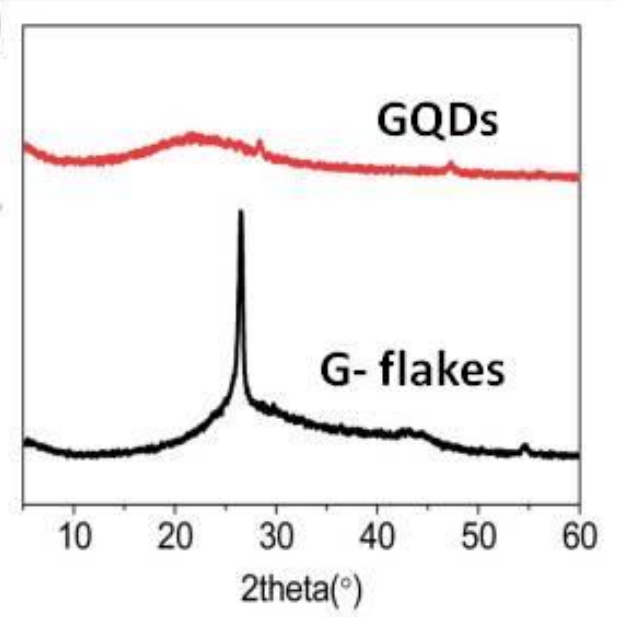

Figure 6: Transmission electron microscopy (TEM) and X-ray diffraction (XRD) examples: (A) TEM images of CQDs formed using ribonuclease $\mathrm{A}$ as a biomolecular templating agent under microwave irradiation (reprint with permission of Ref. ${ }^{67}$ ) and GQDs synthesized using pristine few-layer graphene flakes as a precursor (reprint with permission of Ref. ${ }^{66}$ ); (B) XRD patterns of CQDs (hydrothermal decomposition of 4-aminophenylboronic acid) ${ }^{40}$ and GQDs (reprint with permission of Ref. ${ }^{66}$ )

X-ray diffraction (XRD) allows accurate information to be obtained on the crystallinity of the carbon-based quantum particles (Figure 6B). The XRD pattern of graphene flakes appear as a strong signal at $2 \theta=26.5^{\circ}$ and becomes weak in the case of the formation of GQDs, indicating 
that GQDs are thinner than graphene flasks owing to the further exfoliation of few-layers graphene. The broad peak centered at $2 \theta \sim 21.9^{\circ}(\mathrm{d}=0.41 \mathrm{~nm})$ may result from the $\pi-\pi$ stacking of GQDs. No peaks are observed in the $2 \theta$ region of $5-20^{\circ}$, evidencing that the GQDs are different from GO bearing less oxygen functions. ${ }^{66}$ The XRD diffractogram of CQDs formed from 4-aminophenylbenzene displays a broad diffraction peak at around $21.73^{\circ}$ corresponding to an interlayer spacing of $0.42 \mathrm{~nm}$ being larger than that of bulk graphite, indicating poor crystallization and amorphous character of the structures (Figure 6B). ${ }^{40}$

All carbon-based quantum particles possess different surface functions, needing their evaluation using standard characterization methods such as X-ray photoelectron spectroscopy (XPS), Fourier transformed infrared (FTIR), Raman spectroscopy, as well as sometimes elemental analysis, nuclear magnetic resonance (NMR) or matrix-assisted laser desorption ionization time-of-flight (MADLI-TOF) to determine the general structure. The high resolution $\mathrm{C}_{1 \mathrm{~s}}$ XPS spectrum of CQDs prepared hydrothermally from 4-aminophenylboronic acid, ${ }^{40}$ depicts bands of $\mathrm{C}-\mathrm{C}(283.9 \mathrm{eV}), \mathrm{C}-\mathrm{C} / \mathrm{C}-\mathrm{H}(285.0 \mathrm{eV}), \mathrm{C}=\mathrm{O} / \mathrm{C}-\mathrm{N}(287.3 \mathrm{eV})$ and $\mathrm{O}-$ $\mathrm{C}=\mathrm{O}(290.3 \mathrm{eV})$ functions. The band at $399.6 \mathrm{eV}$ in the $\mathrm{N}_{1 \mathrm{~s}}$ high resolution XPS spectrum indicates the presence of amine groups. The $\mathrm{C}_{1 \mathrm{~s}}$ XPS spectrum GDQs formed by cutting pristine graphene flakes under electrochemical redox reaction in ionic liquid/water electrolyte under reverse potential ${ }^{66}$ can be deconvoluted into bands assigned to $\mathrm{C}-\mathrm{C} / \mathrm{C}-\mathrm{H}(285.0 \mathrm{eV}), \mathrm{C}-$ $\mathrm{O}(286.1 \mathrm{eV}$, such as $\mathrm{C}-\mathrm{OH}$ or $\mathrm{C}-\mathrm{O}-\mathrm{C}), \mathrm{C}-\mathrm{N}(287.2 \mathrm{eV})$, and $\mathrm{O}-\mathrm{C}=\mathrm{O}(288.5 \mathrm{eV})$ (Figure 7A). The $\mathrm{N}_{1 \mathrm{~s}}$ band indicates the presence of two types of $\mathrm{N}$ atoms. The peak at $400.2 \mathrm{eV}$ can be assigned to the $\mathrm{N}$ of pyrrolic $\mathrm{N}$ (the imidazolium group) adsorbed or located at the graphitic edge. Quaternary $\mathrm{N}(401.2 \mathrm{eV})$ atoms may be thought to be incorporated into the graphene layer and substitute carbon atoms within the graphene plane. ${ }^{66}$ 
(A)
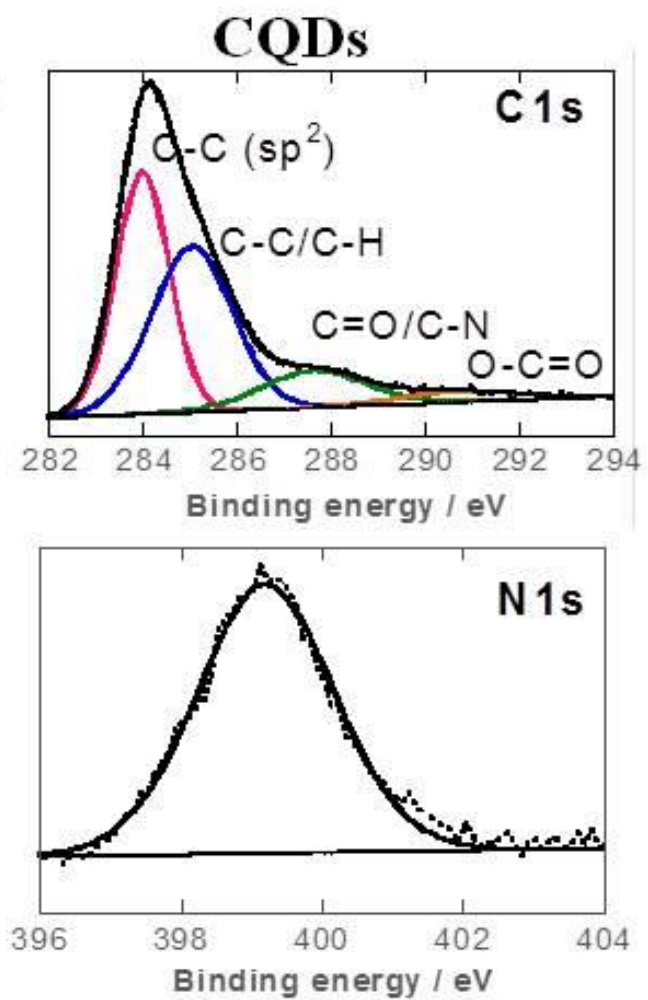

(B)

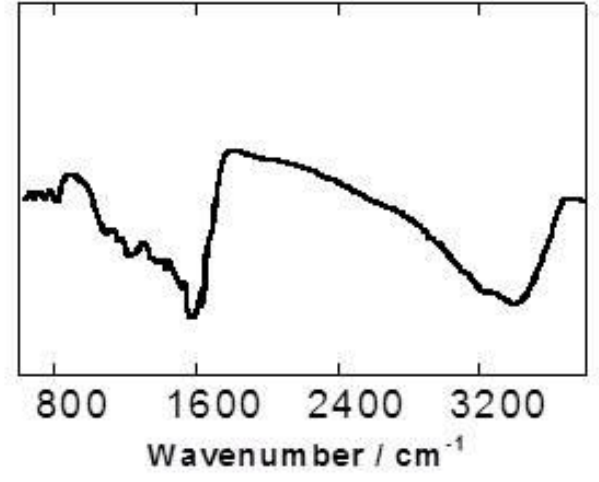

(C)

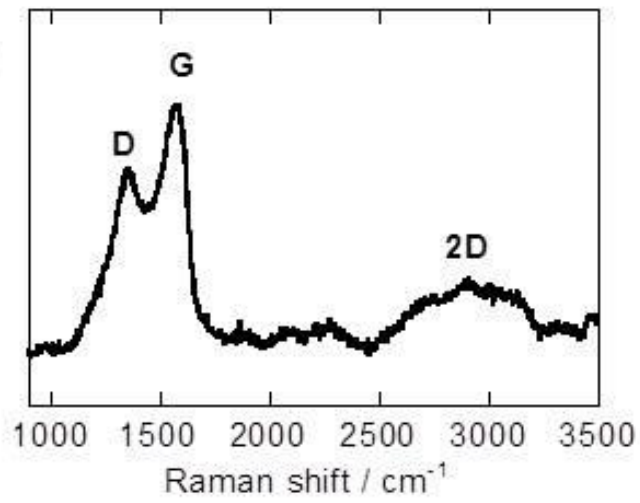

GQDs
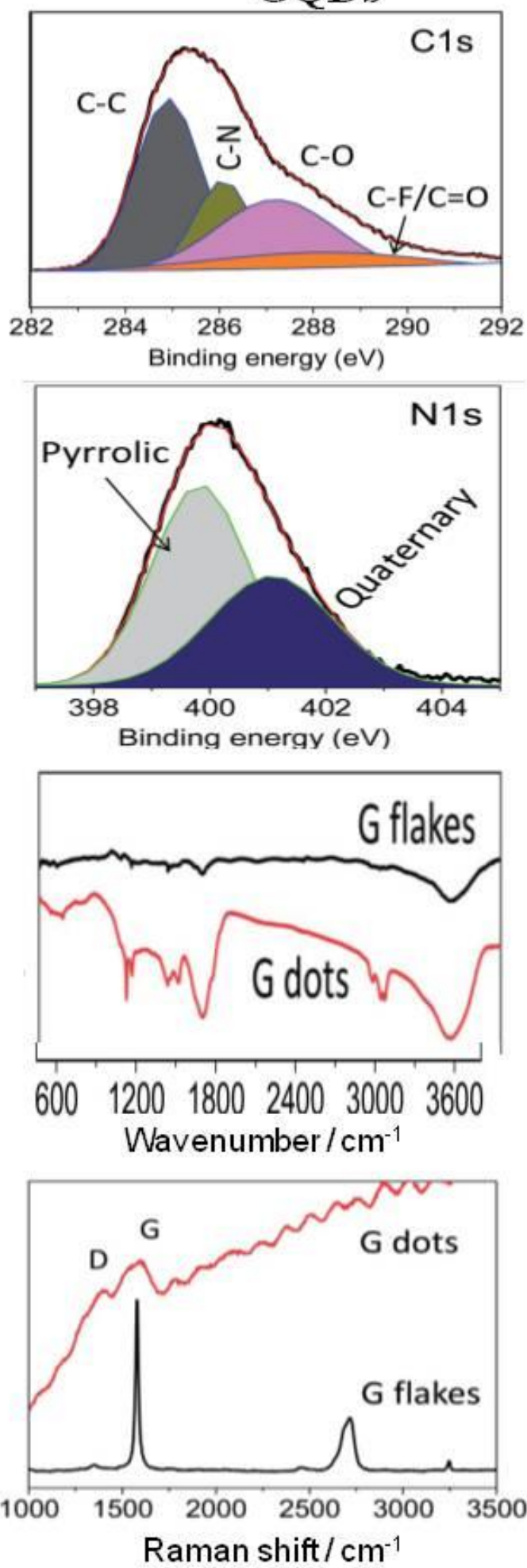

Figure 7: Chemical characterization: (A) XPS bands of CQDs (hydrothermal synthesis using 4-aminophenylboronic acid) ${ }^{40}$ and GQDs (formed by cutting pristine graphene flakes through electrochemical charging (reprint with permission from Ref. ${ }^{66}$ ); (B) FTIR images and (C) Raman images of these CQDs and GQDs. 
Information about the chemical composition can also be obtained from Fourier-transform infrared spectroscopy (FTIR). The FTIR spectrum of synthesized GQDs by electrochemical method displays strong bands at around 3425, 1720 and $1645 \mathrm{~cm}^{-1}$ associated with the vibrations of hydroxyl $(-\mathrm{OH})$, carbonyl $(\mathrm{C}=\mathrm{O})$ and graphitic $(\mathrm{C}=\mathrm{C})$, respectively. The band $1078 \mathrm{~cm}^{-1}$ is related to alkoxy groups (O-C-O) present on the GQDs (Figure 7B). The spectrum reflects that GQDs have many oxygenated functional groups on their surface. The FTIR spectrum is also significantly different from that of the initial graphene flakes, with a weak adsorption band at around $3425 \mathrm{~cm}^{-1}$. The FTIR spectrum of CQD BA depicts similar features: distinct strong bands at $3465 \mathrm{~cm}^{-1}$ (OH vibration) and $1618 \mathrm{~cm}^{-1}(\mathrm{C}=\mathrm{O})$ with additional weak bands attributed to graphitic $\mathrm{C}=\mathrm{C}\left(1645 \mathrm{~cm}^{-1}\right), \mathrm{O}-\mathrm{C}-\mathrm{O}\left(1078 \mathrm{~cm}^{-1}\right)$ and $\mathrm{B}-\mathrm{O}$ $\left(1090 \mathrm{~cm}^{-1}\right)$ stretching and deformation vibration modes of the boroxol bond of the boronic acid moieties. ${ }^{127}$ The presence of $\mathrm{C}-\mathrm{N}$ is seen at $1400 \mathrm{~cm}^{-1}$.

Raman analysis of these CQDs and GQDs display both carbon characteristic D and G bands at around 1350 and $1570 \mathrm{~cm}^{-1}$, respectively. The intensity ratio of the $\mathrm{D}$ band, related to the presence of $\mathrm{sp}^{3}$ defects, and $\mathrm{G}$ band, related to in-plane vibration of $\mathrm{sp}^{2}$ carbon, $\left(\mathrm{I}_{\mathrm{G}} / \mathrm{I}_{\mathrm{D}}\right)$ is a measure of the disorder within the nanostructures. The Raman spectrum of CQDs comprises in addition a broad band at $2996 \mathrm{~cm}^{-1}$ corresponding to $2 \mathrm{D}$ of graphitic structures with a $\mathrm{I}_{\mathrm{D}} / \mathrm{I}_{\mathrm{G}}$ ratio of $\approx 1.24$. In the case of graphene flakes used for the formation of GQDs, the weak D and strong $G$ bands indicate the presence of little-defective graphene flakes (Figure 7C). The increased $I_{D} / I_{G}$ ratio $\left(I_{G} / I_{D} \approx 0.6\right)$ of the formed GQDs suggests the formation of more defective materials.

\subsection{Optical properties}

Despite the diversity of carbon-based quantum dots, these nanomaterials possess some similar optical properties in terms of absorption and fluorescence. Outlining the common optical properties rather than considering specific examples, UV/Vis absorption spectra typically show peaks in the UV region with a tail extending to the visible. The absorption located between 230-270 is generally attributed to $\pi-\pi^{*}$ transition of $\mathrm{C}=\mathrm{C}$. A peak/shoulder located at 300-330 nm is assigned to $\mathrm{n}-\pi^{*}$ transition of $\mathrm{C}=\mathrm{O}$.

One of the most exciting properties of CQDs and GQDs is the size-dependent photoluminescence (PL) (Figure 8A), a feature of quantum confinement, and surface defects as discussed in some details above and some excellent reviews. ${ }^{7,68}$ The fluorescent quantum yield (QY) is rather small, often less than $10 \%$. While the preparation of carbon-based quantum dots with high QYs is still a challenge, a better understanding of the mechanism at hand helps in advancing this field. Eda et al. reported the presence of isolated $\mathrm{sp}^{2}$ clusters within the carbon-oxygen $\mathrm{sp}^{3}$ matrix as the origin of photoluminescence in GQDs with the energy gap varying with the $\mathrm{sp}^{2}$ cluster size. ${ }^{69}$ The PL is thus dictated by the nature and size of the $\mathrm{sp}^{2}$ domains and surface functions associated. Kim et al. showed for example that the peak energy of the PL spectra decreases as the size of GQD changes from 5 to $35 \mathrm{~nm} .{ }^{27}$ Nevertheless size-dependent PL might not tell the whole story. Chemical functionalization is another effective means of tailoring the electronic characteristics of carbon-based quantum 
particles, due to the remarkable edge effects. Chemical modification using molecules with strong electron-donating or electron-accepting abilities cause a remarkable PL wavelength shift due to the marked changes to the electronic characteristics of the nanoparticles. The possibility to tune the emission wavelength of GQDs from the blue to orange by controlling the degree of amine functionalization has been demonstrated. ${ }^{36}$ Indeed, the HUMO/LUMO levels can be tuned by adjusting the strength of the orbital interactions with different nitrogencontaining groups and adjacent groups. Substitution with electron-donating character raises HOMO to a higher energy, causing narrowing of the energy gap. Nitrogen substituted aromatics induce additional energy level between the LUMO and HUMO, markedly narrowing the band gap.

Up-conversion photoluminescence (UCPL) is another attractive property of CQDs. Sun et al. reported that CQDs are strongly emissive in the visible region under irradiation by a femtosecond pulsed laser in the NIR. ${ }^{70}$

(A)

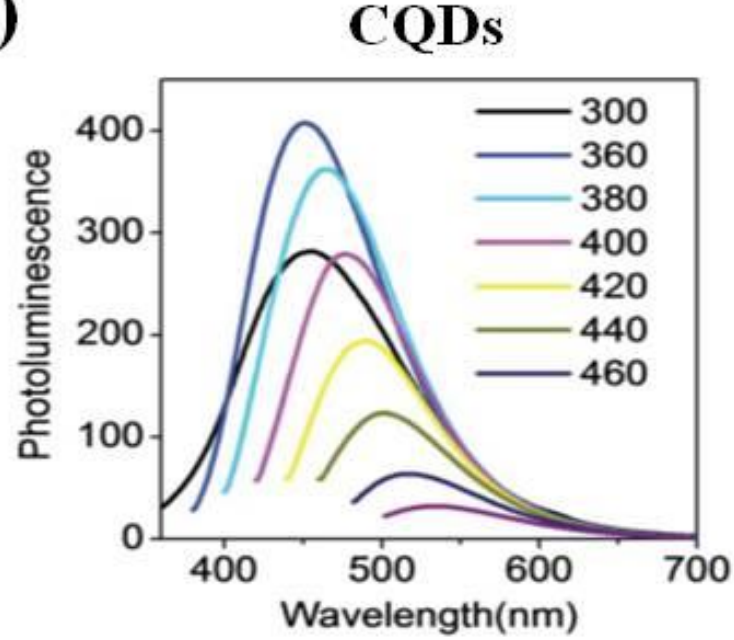

\section{GQDs}

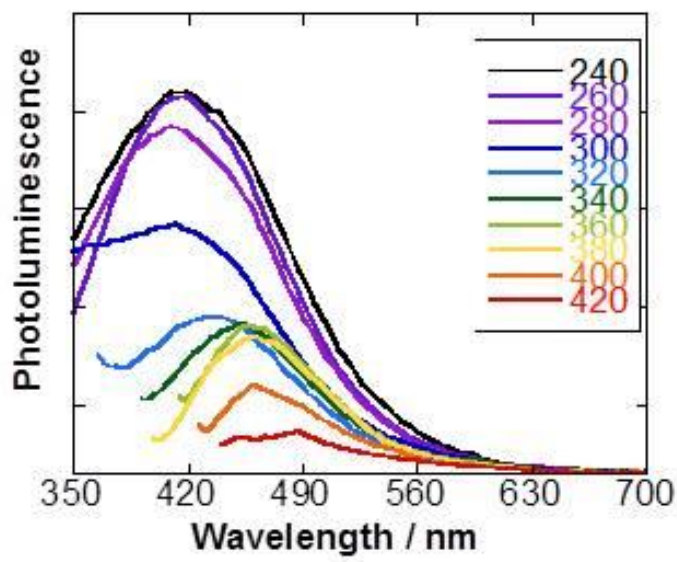

(B)

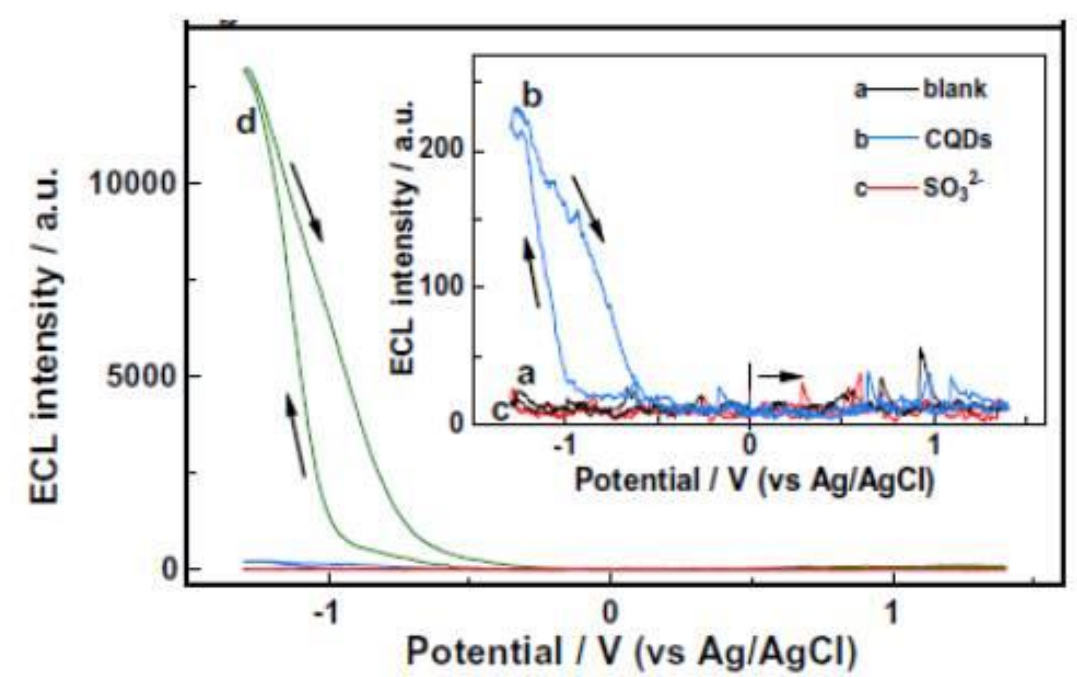


Figure 8: Optical aspects: (A) Photoluminescence spectra at various excitation wavelengths of CQDs ${ }^{40}$ and GQDs (reprint with permission of Ref. ${ }^{66}$ ), (B) ECL potential curve in PBS (i), CQDs (ii), $\left(\mathrm{Na}_{2} \mathrm{SO}_{4}\right)$, (iii) and, $\mathrm{CQDs}+\mathrm{Na}_{2} \mathrm{SO}_{4}$ (iv) (reprint with permission of Ref. ${ }^{71}$ ).

CQDs show next to photoluminescence also electrochemiluminescence (ECL), based on the conversion of electrical energy into radiative emission. ${ }^{72}$ Classical ECL materials include luminol or $\mathrm{Ru}(\mathrm{bpy}) 3_{3}{ }^{2+}$. CQDs are a new class of ECL luminophores where the ECL characteristics are determined by the amount of abundant surface traps, functional groups and other surface passivation factors. Indeed, different to PL, ECL is mainly associated with surface-state transition in the nanoparticles. Similar to metal-based quantum dots, CQDs follow the cathodic ECL mechanism using $\mathrm{K}_{2} \mathrm{~S}_{2} \mathrm{O}_{8}$ as reactant. This mechanism is explained as follows: the functional oxygen-groups in CQDs facilitate the electro-generation of CQDs ${ }^{-}$ radical, oxidizing $\mathrm{SO}_{4}{ }^{--}$radicals produced by the electrochemical reduction of $\mathrm{S}_{2} \mathrm{O}_{8}{ }^{2-} \cdot{ }^{-}$B Both radicals interact via electron-transfer annihilation to form the excited state of CQD* (Figure 8B). Finally, the excited CQD* returns to the ground state via a radical pathway emitting a photon. This property is mainly used for sensing as exemplified by the electrochemiluminescent immunosensor developed by Li et al. $^{73}$

Tuning the emission spectrum from the green to the near infrared has been achieved by Sun et al using surface passivation. ${ }^{74}$ Doping fluorescent CQDs with heteroatoms is another feasible way to tune the intrinsic properties of CQDs and GQDs. ${ }^{8}$ Since the first report on nitrogen (N) doped GQDs in 2012, large efforts are being put on exploring different procedures for making new types of doped CQDs. The exact mechanism of the fluorescence shift in doped CQDs is still under investigation and further discovers are expected in the close future. Nevertheless, doped CQDs have found already applications in the biomedical field as multicolor nanoprobes that can be excited with different excitation wavelength. ${ }^{2}$

\subsection{Redox Properties of Carbon-Based Quantum Particles}

In contrast to the understanding of metallic and semiconducting carbon nanotube materials, ${ }^{75}$ work on the electronic and electrochemical properties of well-defined CQDs and GQDs for electrochemical applications is still quite underdeveloped. As already exemplified with ECL, carbon-based nanomaterials are of considerable interest in electrochemical applications, where properties such as (i) electronic conductivity and sites for electron transfer, (ii) high surface area, (iii) photo-active reaction centers, and (iv) catalytic reaction sites provide opportunities for new technologies.

Carbon-based quantum materials often exhibit only some of these properties. Lim et al. compared electrochemical performance of CQDs and GQDs by using redox probes including potassium ferro/ferricyanide. Based on the results obtained from cyclic voltammetry studies, CQDs somehow impart improved electron transfer characteristics using ferro/ferricyanide as 
redox couple (Figure 8A) ${ }^{76}$ The importance of surface functionalities on the electrochemical response has been demonstrated by Marken and co-workers in several papers. ${ }^{77-79}$ The hydrothermal conversion of chitosan leads to electrochemically inactive carbon nanomaterials, while "hydrothermal wrapping" of a negatively charge Pd-carbon core shell particles with chitosan gave a positively charged nanocomposite (Figure 8B). ${ }^{77,} 79$ Upon further modification with anthraquinone moieties via diazonium coupling, the electrochemical reactivity became $\mathrm{pH}$ dependent with a good electrical conduction of charges only achieved in acidic conditions.
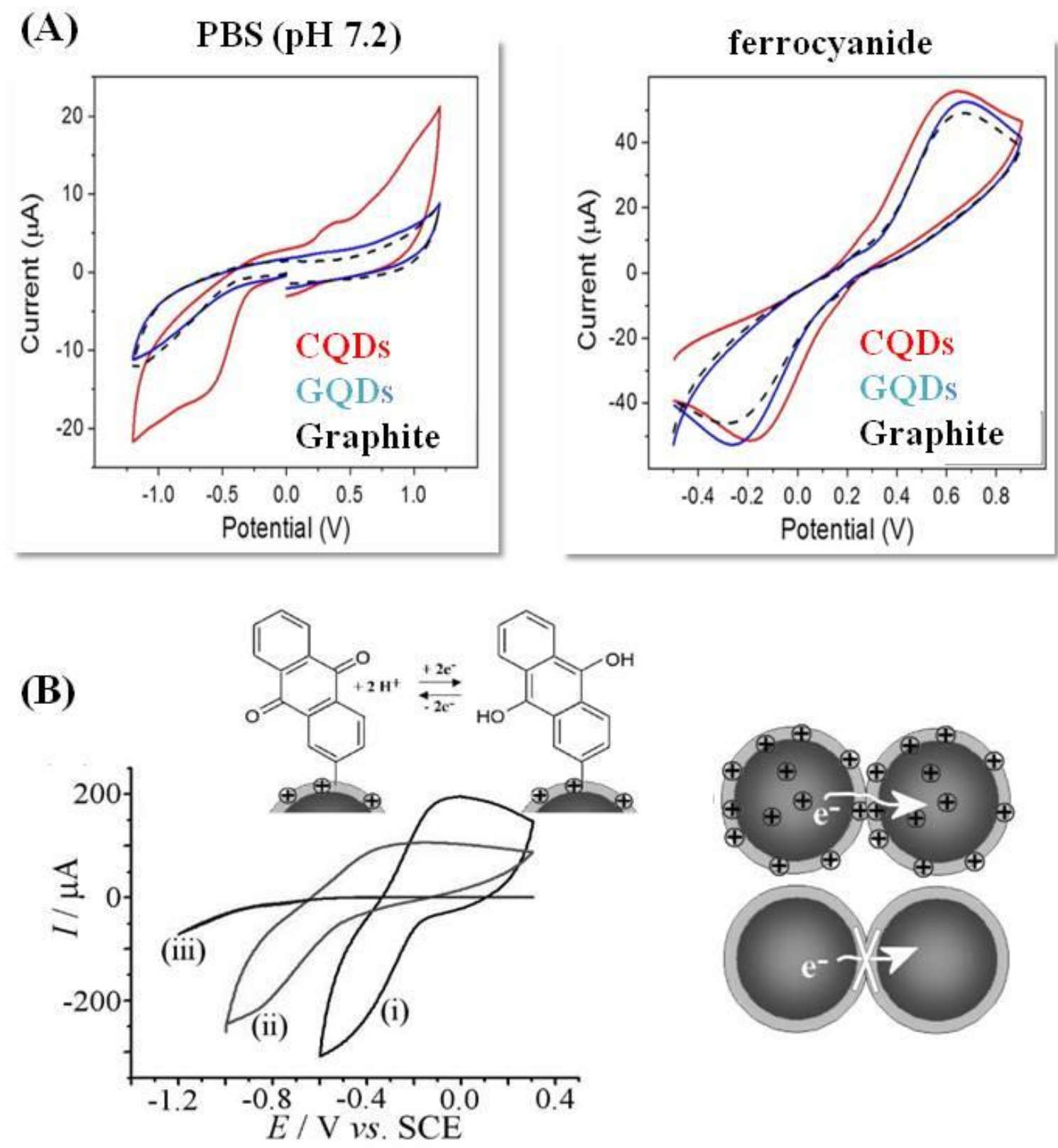

Figure 9. Electrochemical properties and electrocatalytic activity of carbon-based quantum particles: (A) Comparison of cyclic voltammograms of CQDs, GQDs and bare 
basal plane pyrolytic graphite electrode in PBS ( $\mathrm{pH}$ 7.2) and potassium ferro/ferricyanide (10 $\mathrm{mM}$ ), scan rate $=100 \mathrm{mV} \mathrm{s}^{-1}$ (reprint with permission from Ref. ${ }^{76}$ ); (B) Cyclic voltammograms for the reduction of covalently bound anthraquinone at $0.05 \mathrm{mg}$ carbon nanoparticles modified ITO $\left(1 \mathrm{~cm}^{2}\right)$ electrode when immersed in PBS at $\mathrm{pH} 2$ (i), pH 7 (ii), and $\mathrm{pH} 12$ (iii) (together with the anthraquinone-anthraquinol redox process (reprint with permission from Ref. ${ }^{79}$ )

A general concept to improve the electrochemical properties of CQDs is their embedment and combination with various materials including graphene, graphene oxide, carbon nanotubes, metallic nanoparticles, metal oxides or conducting polymers. Such nanocomposites find wide applications in electrocatalysis as discussed later CQDs doping is another means for improving the electrochemical activity. ${ }^{2,80}$ As an example, films of nitrogen-doped/graphene hybrid material exhibits a clear catalytic response for methanol in aqueous $0.1 \mathrm{M} \mathrm{KOH}$. The electrochemical reduction of carbon dioxide $\left(\mathrm{CO}_{2}\right)$ on different carbon nanomaterials

including CQDs has been reviewed by Duan and coworkers. ${ }^{81} \mathrm{~N}$ - and P-codoped CQDs were suggested to offer promising performance with a range of products being obtained under relatively mild conditions. ${ }^{151}$ A broader range of applications of carbon quantum particles in electroanalysis has emerged (vide infra).

\subsection{Cell Viability and Toxicity}

In contrast to metallic nanoparticles, carbon-based nanomaterials are less cytotoxic in general, mainly due to their hydrophilic character and the use of green reagents for their synthesis without the use of any metal catalysts. For example, CQDs obtained from green tea exhibited cell viabilities $>95 \%$ towards pig kidney cell line even at concentrations as high as $1.8 \mathrm{mg}$ $\mathrm{mL}^{-1} .82$

Some of us investigated the cytotoxicity of boronic- and amine-modified CQDs using the Uptiblue ${ }^{\circledR}$ cell-viability assay on A549 and Vero cells (Figure 10A) and found that all nanostructures are not cytotoxic at concentrations up to $300 \mu \mathrm{g} \mathrm{mL}^{-1}$. Fluorescence imaging (Figure 10B) further confirmed the relatively low amounts of CQDs accumulation in vivo without causing any notable damage to the organs. Yan et al. described recently the effect of surface charge on the cytotoxicity and uptake of CQDs, formed from different ratios of citrate and spermidine as starting materials, in human umbilical cord derived mesenchymal stem cells. ${ }^{83}$ All particles at concentrations below $50 \mu \mathrm{g} \mathrm{mL} \mathrm{mL}^{-1}$ were nontoxic with slightly positively charged CQDs showing higher cell uptake efficiency than negatively charged ones. This low toxicity of CQDs derived from glucose and surface stabilized with ethylenediamine was recently confirmed using zebra fish as an in vivo model. ${ }^{84}$

Zboril and co-workers presented a comprehensive in vitro cytotoxic study on mouse fibroblasts (NIH/3T3) of CQDs differing in surface functionalisation ${ }^{85}$ namely negative charged CQDs due to the presence of carboxylic acid groups, neutral polyethyleneglycol modified dots CQDs and polyethylenimine coated ones and thus positively charged particles. It was suggested that the neutral particles are the most promising for biological applications as they do not induce any abnormalities in cell morphology up to concentrations of $300 \mu \mathrm{g} \mathrm{mL}^{-1}$. Negatively charged CQDs on the other hand stimulated proliferation and led to 
higher oxidative stress; however they did not enter the cell nucleus. In contrast, positively charged CQDs were the most cytotoxic, entering into the cell nucleus and inducing the largest changes in G0/G1 phase of cell cycle at concentrations of around $100 \mu \mathrm{g} \mathrm{mL}^{-1}$. All the available data indicate that the surface function of CQDs is determining cell viability with positively charged CQDs being more cytotoxic than others.

Using in vivo NIR-fluorescence imaging of intravenously (via the tail vein) injected CF790labeled CQDs in five-week-old male Swiss nude mice, an intense fluorescence signal was observed in the bladder after only $2.5 \mathrm{~h}$ post-injection. This indicates that intravenously injected CQDs were removed from the body of mice through an excretion pathway, especially via urine. This is in line with other studies where CQDs were injected intravenously (440 $\mu \mathrm{g}$ in $200 \mathrm{~mL}$ ) and found to be removed from the body of mice through urine, as evidenced from dissected organs such as kidneys and liver. ${ }^{86}$

(A)

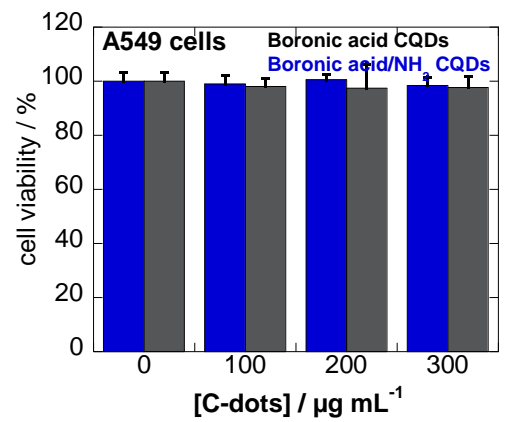

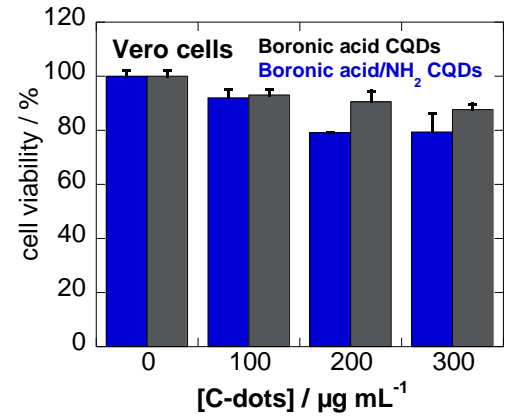

(C)
(B)

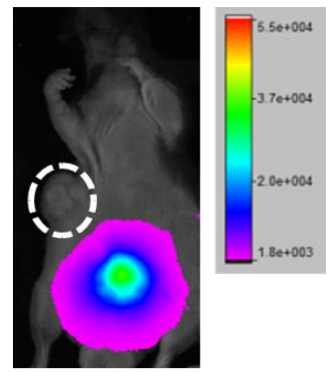

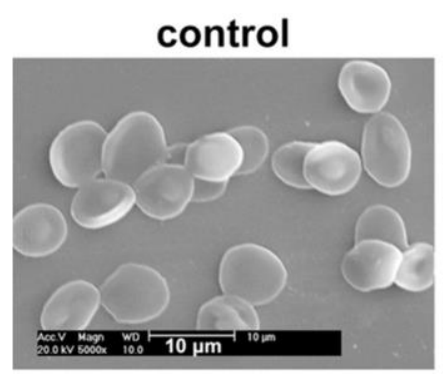

$1 \mathrm{mg} / \mathrm{mL}$

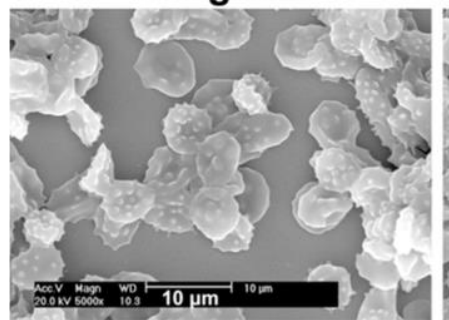

$0.01 \mathrm{mg} / \mathrm{mL}$

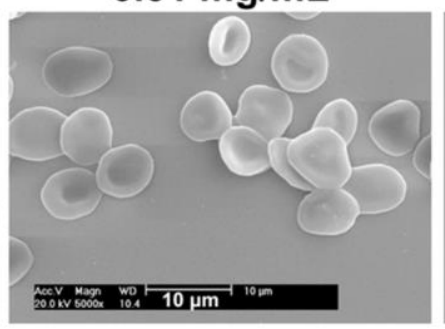

$5 \mathrm{mg} / \mathrm{mL}$

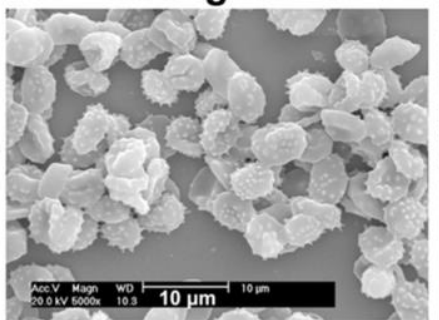

$0.1 \mathrm{mg} / \mathrm{mL}$

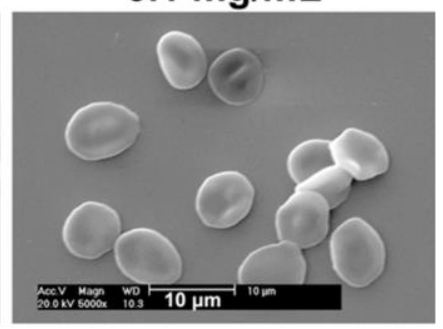

$10 \mathrm{mg} / \mathrm{mL}$

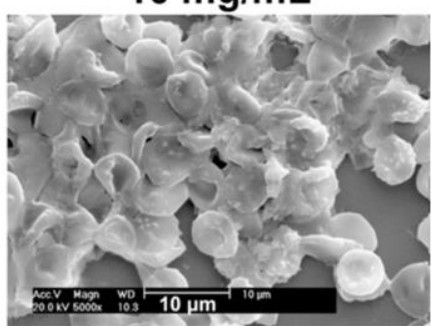

Figure 10. Cell viability and bio-distribution: (A) Cell viability of A54 and Vero cells incubated for $72 \mathrm{~h}$ with different concentrations of functional CQDs. Viability was evaluated by using Uptiblue ${ }^{\circledR}$ assay $^{40}$; (B) Bio-distribution of CF790-labeled CQDs after $2.5 \mathrm{~h}$ post injection (unpublished results); (C) Aggregation of red-blood cells in the presence of different 
concentrations of the fluorescent carbon dots as observed with SEM (reprint with permission from Ref. $^{87}$ );

Determination of the hemolysis activity of carbon-based quantum particles has in addition become of importance to give a full toxicity profile of these nanostructures. ${ }^{87}$ To date, little information is available about the hemocompatibility of carbon-based quantum particles, something which could impede their development from laboratory to bedside. Hemolysis may occur when the red blood cell membrane is ruptured and releases hemoglobin into blood plasma which may lead to anemia, hypertension, renal toxicity, etc.. According to ASTM E2524-08 standard (Standard test method for analysis of hemolytic products of nanoparticles), less than $5 \%$ hemolysis is permissible for biomaterials. Fluorescent carbon quantum dots prepared by hydrothermal carbonization of $\alpha$-cyclodextrin were investigated lately by Li et al for their hemolyis activity. ${ }^{87}$ A concentration-dependent effects on the blood components was observed (Figure 10C) with concentrations of $\leq 100 \mu \mathrm{g} \mathrm{mL}^{-1}$ having few adverse effects on the blood components, while at higher doses, the structure and function of the blood components were impaired, causing morphological disruptions and lysis of red blood cells, interference in the local microenvironments of fibrinogen, activation of the complement system, and disturbances in the plasma and whole blood coagulation function in vitro. Platelets were only activated at low concentrations. Intravenous administration of the carbon dots at doses up to $50 \mathrm{mg} / \mathrm{kg}$ did not impair the blood coagulation function.

\section{Surface Functionalization of carbon-based quantum particles}

Surface modification is a powerful approach for tuning the surface properties of a material for the selected application. The type of surface functional groups present on carbon-based quantum particles depends on the precursor used as well as on the reaction conditions (Table 1). Generally, surface functions including carboxylic acid, hydroxyl and/or amine groups are present on the surface of CQDs using molecular precursors such as citric acid, glucose or polyethylenediamine (PEI) to give some examples. The hydrophilic nature of the resulting particles simplifies the preparation of homogenized suspension, necessary for most applications and particularly important for biomedical orientated issues.

Next to the introduction of functionality during the synthetic process, post-functionalization, involving generally covalent modification steps are routinely performed (Figure 11A). The majority of CQDs are rich in oxygen-containing groups and surface passivation via covalent bonding of amine-containing agents is a commonly used method not only to improve the photoluminescence properties of CQDs, but also to attach $\mathrm{NH}_{2}$-containing agents such as antibiotics. ${ }^{88}$ Some of the modified CQDs revealed excellent chelating capability for metal ions. Chen et al. functionalized COOH-terminated CQDs with 1,4,8,11tetraazacyclotetradecane cyclam via amide bond formation to obtain highly water soluble particles with superior affinity for $\mathrm{Cu}^{2+} .89$

Since CQDs are typically synthesized by treating with urea or PEI as co-reactants, the particles are also rich in amino groups, allowing covalent bond formation with $\mathrm{COOH}$-rich structures. Kim et al. grafted R6G-COOH onto CQDs- $\mathrm{NH}_{2}$ and used it for the fluorescence 
based detection of $\mathrm{Al}^{3+} \cdot{ }^{90}$ Glutathione-modified gold nanoclusters coupled to CQDs- $\mathrm{NH}_{2}$ allowed the detection of highly reactive oxygen species. ${ }^{91}$

Next to carboxylic and amine groups, the surface hydroxyl groups of CQDs represent an interesting family of functional groups for surface functionalization. Encapsulating CQDs into silica shells results in particles with minimized particle aggregation and nonspecific binding.

(A)

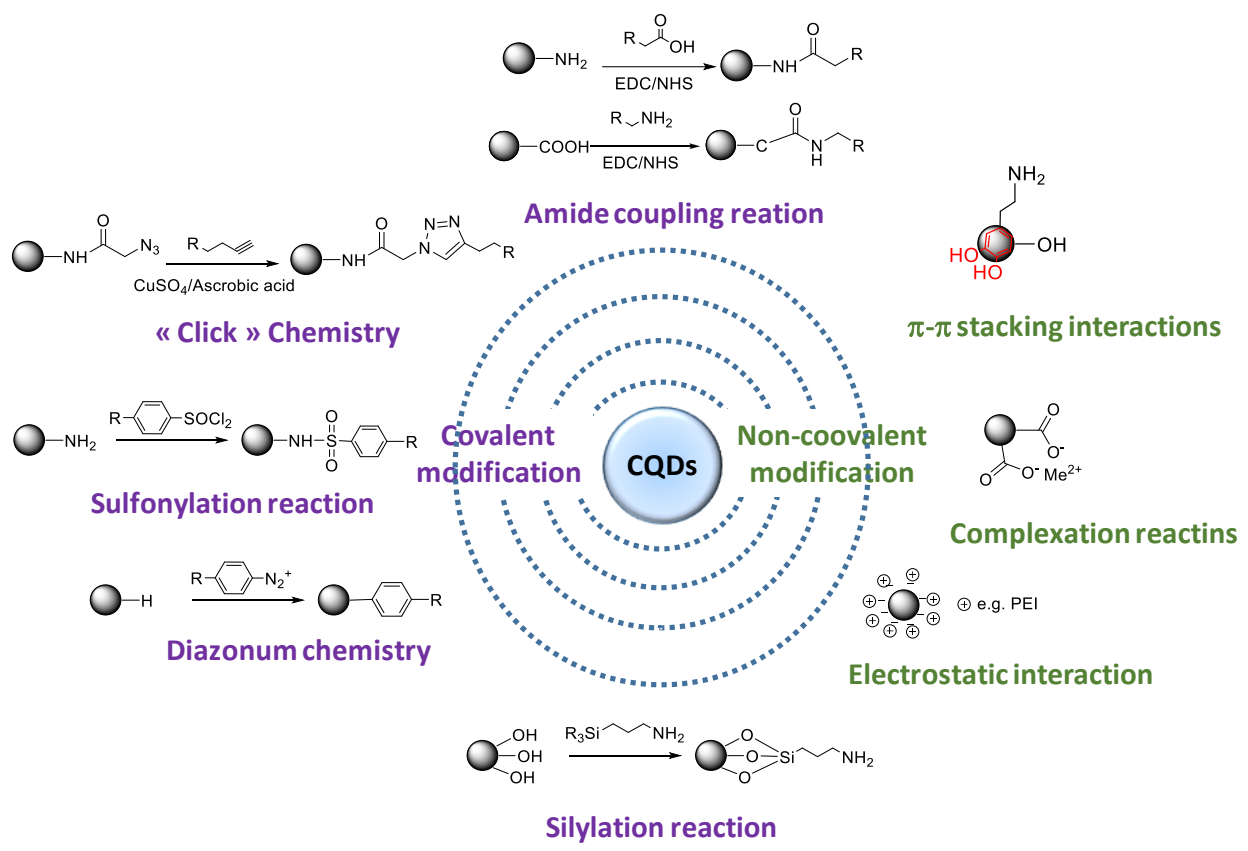

(B)

(C)

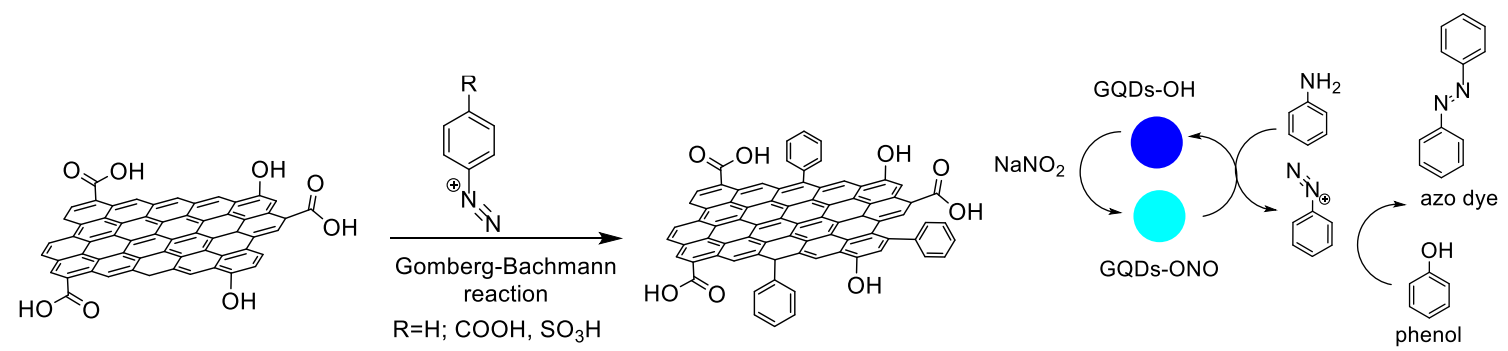

Figure 11: Surface modification strategies of carbon-based quantum nanoparticles: (A) Covalent and non-covalent strategies; (B) Schematic illustration of particle modification the using Gomberg-Bachman reaction, ${ }^{56}$ (C) Preparation of GQDs-ONO and its application as highly active catalyst for the synthesis of azo-dyes ${ }^{92}$.

Diazonium-based chemistry has been widely used for the functionalization of various substrates including more recently carbon-based materials. ${ }^{93}$ This versatile chemistry has been extended to carbon-based quantum particles notable GQDs to incorporate aryl groups onto their surface. The GQDs were prepared from natural graphite powder using acid $\left(\mathrm{H}_{2} \mathrm{SO}_{4} / \mathrm{HNO}_{3}\right)$ treatment at $100{ }^{\circ} \mathrm{C}$, followed by thermal deoxidization at $300^{\circ} \mathrm{C}$ under an argon atmosphere. The thermal treatment was performed to remove most of the oxygen functional groups on the GQDs surface and increase the active sites for C-C coupling. In this 
process, the diazonium salt was generated in situ by the reaction of $\mathrm{NaNO}_{2}$ with various arylamines (aniline, 4-aminobenzoic acid, sulfanilic acid or 5-aminonaphthalene-1-sulfonic acid) in $\mathrm{HCl}$ aqueous solution at low temperature $\left(0-5^{\circ} \mathrm{C}\right)$, followed by addition of $\mathrm{NaOH}$ at this temperature to produce the corresponding diazotate. Subsequent reaction of the diazotate with GQDs at $90^{\circ} \mathrm{C}$ gave the corresponding aryl-modified GQDs (Figure 11B). Interestingly this chemical functionalization enhanced the photoluminescence (PL) intensity of the GQDs and improved their PL stability in a wide $\mathrm{pH}$ window of 1-11.

Another more recent functionalization strategy is that developed by Valizadeh et al. ${ }^{92}$ for the preparation of nitrite-functionalized GQDs (Figure 11C). The reaction of GQDs with $\mathrm{NaNO}_{2}$ was conducted in the absence of mineral acids to generate nitrite-functionalized GQDs. These modified GQDs were employed as an effective nitrosonium ion source for diazotization of various arylamines and subsequent synthesis of azo-dyes.

In contrast to covalent functionalization strategies, non-covalent modification based on electrostatic interaction, complexation or $\pi-\pi$ stacking interactions are also possible. The surface charge of CQDs can be easily tuned positively or negatively by choosing the appropriate starting materials. Jin et al. assessed the electrostatic interaction between positively charged PEI and negatively charged CQDs, formed from natural carrot as carbon source, for the detection of $\mathrm{S}^{2-.94}$

\section{Applications}

The different and unique physicochemical properties and the possibility to synthesize a large panel of carbon-based nanostructures have made them of interest for applications in several different fields ranging from sensors, catalysis, to energy and medicine. Some recent review articles are devoted to some of these exciting applications. ${ }^{6,16,68,95}$ Different to some of them, the review aims to act as a bridge between optical and electrochemical aspects of carbonbased quantum particles, both of which are associated with the electronic nature of carbonbased quantum particles. The emphasis will be on fluorescence and electrochemiluminescent sensors to continue outlining the use of carbon-based quantum particles in electroanalysis. The use of carbon-based quantum particles in electroanalytic formats is lagging behind that of optical sensing formats. However, integration of carbon-based quantum particles into electrochemical detection schemes as well as the use of a large variety of carbon-based quantum particles composite nanomaterials has largely improved detection limits and detections scopes. Keeping the definition, the large field of carbon nanotubes and graphene nanosheets as well as diamond electrochemistry is not considered here. ${ }^{95}$

\section{1. Chemical and biological sensing}

Over the years, next to CQDs based fluorescence (Table 1), electrochemical (Table 2) sensors have gained tremendous interest due their interesting photo-induced electron transfer and electrochemical charge transfer properties of CQDs. To this has to be added hybrid CQDs such as those made with metallic nanoparticles due to their excellent static and dynamic fluorescence quenching and electrocatalytic performance. A non-enzymatic electrochemical sensor based on CQDs/octahedral cuprous oxide $\left(\mathrm{Cu}_{2} \mathrm{O}\right)$ hybrid proved to be efficient for the detection of glucose and hydrogen peroxide with a detection limit of $8.4 \mu \mathrm{M}$ and a liner range 
up to $4.3 \mathrm{mM} .^{96}$ Diabetes is a chronic disease characterized by high glucose content in blood caused either by the absence of insulin secretion or by the inability of the body cells to respond properly to the secreted insulin. To avoid major complications such as kidney failure, limb amputation, blindness and coronary artery disease, frequent monitoring of blood glucose has become essential for diabetic patients. For the majority of healthy individuals, normal blood sugar levels are between 4-7.8 mM, while for people with type 1 or type 2 diabetes the blood sugar can rise up to $11 \mathrm{mM}$. As the performances of such electrochemical sensors are often compared to optical ones, some details of fluorescence-based sensors using CQDs will be given first.

\subsubsection{Photoluminescence sensors}

Fluorescence probes serves as unique detection methods for its simplicity and low detection limit (LOD) and especially bioimaging ability. As the emission spectrum as well as the fluorescence intensity is dependent on the external environment, CQDs and GQDs are ideal nanomaterials for the development of fluorescence based sensors.

\section{Metal ion sensors}

Metal cations like $\mathrm{Zn}^{2+} \mathrm{Cu}^{2+}$ and $\mathrm{K}^{1+}$ play a raft of important signalling roles in our bodies; others such as $\mathrm{Hg}^{2+,}, \mathrm{Cd}^{2+}$ and $\mathrm{Pb}^{2+}$ are toxic pollutants in the environment. The contamination by heavy metals of water is one of the major issues to be faced throughout the world and requires special attention. Excess amount of iron (more than $10 \mathrm{mg} / \mathrm{kg}$ ) causes rapid increase in pulse rate and coagulation of blood in blood vessels, hypertension and drowsiness. The maximum allowed concentration of iron in drinking water is $1.0 \mathrm{mg} \mathrm{l}^{-1}$ according to WHO report. The permissible limit of zinc is $5 \mathrm{mg} \mathrm{l}^{-1}$ and that of cupper in $2 \mathrm{mg}$ $1^{-1}$ Copper not only can accumulate in liver and brain, but contamination of drinking water with high level of copper may lead to chronic anemia. In the case of cadmium, the permissible level is only $0.01 \mathrm{mg} \mathrm{l}^{-1}$. The concentration of inorganic mercury in drinking-water sources is usually less than $0.5 \mu \mathrm{g}^{-1}$ The development of sensing strategies to selectively image these ions inside cells as tools for diagnosing diseases like diabetes and Alzheimers disease and in the environment has thus become timely ${ }^{97}$.

Most of the procedures proposed for metal ions detection are based on fluorescence quenching by metal ions such as the detection of $\mathrm{Hg}^{2+},{ }^{98-101} \mathrm{Cu}^{2+},{ }^{102} \mathrm{Fe}^{3+},{ }^{103} \mathrm{Al}^{3+},{ }^{104}$ and $\mathrm{Ag}^{+} \cdot{ }^{103,104,105-108}$ In this detection scheme, metal ions interact with CQDs via surface bonding, which results in the formation of electron-hole recombination via an electron transfer process and changes the fluorescence intensity, used as measurable response signal. ${ }^{109} \mathrm{CQDs}-\mathrm{COOH}$ interact or example with high affinity with $\mathrm{Hg}^{2+}$ to create new non-radiative hole-pairs, leading to fluorescence quenching. ${ }^{98}$ The presence of nitrogen elements in N-doped CQDs is responsible for improved $\mathrm{Hg}^{2+}$ sensing with a detection limit in the nanoMolar concentration range and large a Stern-Volmer constant between CQDs and $\mathrm{Hg}^{2+}$ of $1.4 \times 10^{7} \mathrm{M}^{-1} .{ }^{100}$ More recently, the selective $\mathrm{Hg}^{2+}$ detection in living cells was achieved using CQDs derived from citric acid and 1,2-ethyldiamine or $\mathrm{N}$-(b-aminoethyl)g-aminoproyl. ${ }^{110}, 111$ The metal ion sensing examples are not limited to $\mathrm{Hg}^{2+}$. 
$\mathrm{Fe}^{3+}$ ions, playing an important role in vivo where abnormal levels can cause severs diseases could be sensed with CQDs, prepared from $p$-phenylenediamine in the presence of nitric acid by hydrothermal reaction, due to selective binding of $\mathrm{Fe}^{3+}$ with carboxyl, ester and hydroxyl groups on the CQDs surface. ${ }^{103}$ Dual-emission fluorescent $\mathrm{Cu}$-doped CQDs were proposed next for multi-analyte sensing including $\mathrm{Fe}^{3+}$. These particles were synthesized via a one-pot solvo-thermal method using 1-(2-pyridylazo)-2-naphtol as precursor and revealed two fluorescence emission peaks at 426 and $488 \mathrm{~nm}$ under excitation at $350 \mathrm{~nm}$ upon interaction with $\mathrm{Cu}^{2+}{ }^{102}$ The addition of $\mathrm{Cu}^{2+}$ to the nanoparticles promotes further $\mathrm{d}$-d transition, with the copper doped particle energy level being lower than that of the initial CQDs excited state, resulting in the formation of a new fluorescence emission band. ${ }^{102}$

\section{Anion sensors}

Anion recognition and anion sensing are of interest as anions play many important roles in medical, biological and environmental areas. ${ }^{112}$ Contrary to the metal ion assays based on fluorescence quenching, many of the anion assays exploit fluorescence enhancement of already quenched CQDs/metal ion complexes. ${ }^{94,113}$ For example, the addition of $\mathrm{S}^{2-}$ leads to the recovery of quenched fluorescence by combining $\mathrm{Cu}^{2+}$ ions with CQDs derived from Carrot juice and modified with PEI and Nile blue. ${ }^{94}$ CQDs/metal ion ensembles were proposed as a triple-channel fluorescent sensor array for the identification of various phosphate anions (e.g. ATP, ADP, AMP, PPi, and Pi). ${ }^{114}$ The selected three ions $\mathrm{Ce}^{3+}$ (in Tris $\mathrm{pH}$ 7.0), $\mathrm{Fe}^{3+}$ (HAc-NaAc, pH 5.0), and $\mathrm{Cu}^{2+}$ (HERS, $\mathrm{pH}$ 7.4) all induce aggregation of the CQDs and fluorescence quenching. Disaggregation or further aggregation of these CQDs/metal ion ensembles occur upon the addition of phosphate anions. The various numbers of phosphate group together with steric hindrance change their affinities to the sensor array and the fluorescence spectrum changes specifically and can be used for their detection and discrimination. ${ }^{114}$

\section{pH sensors}

$\mathrm{pH}$ has great importance in laboratory measurements because a lot of chemical and biological processes are dependent on $\mathrm{pH}$. In modern day research and industrial applications knowing the exact $\mathrm{pH}$ at the exact time is thus of paramount importance. The detection of changes in $\mathrm{pH}$ is also important in living systems. For highly metastatic cancer cells, the $\mathrm{pH}$ measured at the surface is decreased to about 6.7-6.8, while the surrounding external $\mathrm{pH}$ is around 7.4. $\mathrm{pH}$ responsiveness can be integrated into CQDs by increasing the degree of carboxyl groups deprotonation with increasing $\mathrm{pH}{ }^{115}, 116$ The $\mathrm{pH}$ in HeLa cells could be measured by fluorescence using CQDs synthesized by the hydrothermal treatment of citric acid and fuchsine as organic precursors. The CQDs' fluorescence ratio of the dual emission bands, 475 and $545 \mathrm{~nm}$, under single-wavelength excitation changes linearly between $\mathrm{pH} 5.2$ to 8.8 (Figure 12A) ${ }^{117}$ Recently multi-doped carbon dots with ratiometric $\mathrm{pH}$ sensing properties were developed and used to monitor enzyme catalytic reactions. ${ }^{118}$

\section{Chemical sensors}


Generally, chemical sensors are used to detect very small amounts of chemical vapors. It is an active research area based on the need to obtain increasing amounts of data in chemical and food process streams as well as environmental monitoring. Different types of detection elements can be used, one of them being CQDs and GQDs. Currently the examples using carbon-based quantum particles are limited to a handful including the detection of tetracycline, ${ }^{119}$ explosive nitrocompounds, ${ }^{120,}{ }^{121}$ and biothiols ${ }^{122}$ such as amino acids, peptides, proteins, enzymes. Zhou et al. ${ }^{123}$ developed a sensor for dopamine sensing based on polyindole/GQDs molecularly imprinted polymers (PIn/GQDs@MIPs). The proposed sensor exhibits a high sensitivity in the determination of dopamine, which is probably due to the tailor-made imprinted cavities for binding dopamine through hydrogen bonds between amine groups of dopamine and oxygen-containing groups of the novel composite. Magnetic GQDs embedded molecularly imprinted polypyrrole (mSGP) were designed to specifically and effectively capture, pre-concentrate and detect analytes due to the synergy among chemical, magnetic and molecular imprinting properties with the fluorescence properties of GQDs, which are quenched upon analyte recognition. ${ }^{124}$ This multifunctional material enables a rapid, simple and sensitive platform for small molecules detection even in complex media such as seawater without any sample treatment.

\section{Biosensors}

Biosensors are analytical devices that convert a biological response into an electrical signal. The essentially characteristic of a biosensors is that is must be highly specific, independent of physical parameters such as $\mathrm{pH}$ and temperature and should be reusable. Fabrication of an effective biosensors requires multidisciplinary research in chemistry, biology, and engineering. Based on their detection mechanisms, they can be categorized into three main groups: (i) biocatalytic group comprising enzymes, (ii) bioaffinity group including antibodies and nucleic acids, and (iii) microbe based group containing microorganisms as surface ligands. The transducer materials these ligands can be attached are numerous, and CQDs have found to play an important role in the improvement of biosensors performance. The increase demands for simple, cheap, sensitive and selective DNA biosensors are ubiquitous, due to the important role that DNA detection for disease diagnostics. ${ }^{125}$ Carboxylic-rich CQDs were proposed as fluorescent nanoquencher for the detection of nucleic acids based on a homogeneous fluorescent assay. ${ }^{126}$ Carboxylic-rich CQDs, notably citric acid and malic acid derived ones, have been proposed by Pumera and co-workers as a fluorescence sensing platform for DNA, given that single-stranded DNA (ssDNA) molecules adsorb in a completely different pathway to carboxylic-rich CQDs compared to double-stranded DNA (dsDNA). Adsorption of a fluorescently labeled ssDNA onto CQDs resulted in fluorescence quenching. ${ }^{126}$ When the probe undergoes hybridization with its complementary DNA target to form dsDNA, the interactions with the carboxylic-rich CQDs are weakened, leading to fluorescence recovery of the probe.

The increase in bacterial infections and the rise in multi-drug resistant bacteria has resulted in an increased demand for the development of early stage warning systems. Papaya-derived CQDs were successfully applied for the sensing of E. coli O157:H7 with a limit of detection (LOD) of $9.5 \times 10^{4} \mathrm{cfu} \mathrm{mL}^{-1} .127$ 


\subsubsection{Electrochemiluminescence sensors}

Electrochemiluminescence (ECL) is a special form of chemiluminescence, in which the lightemitting chemiluminescent reaction is preceded by an electrochemical reaction. It is thus a means of converting electrical energy into light (radiative energy). Especially nanomaterialsbased ECL has attracted tremendous attention and been extensively employed for the quantitative determination of different types of protein analytes, due to its remarkable features such as high versatility, wide dynamic detection range, low background noise, simple optical setup, and good reproducibility of this technique. CQDs have aroused large interest for electrochemiluminescence (ECL) studies. ${ }^{62,128}$ They are generally used as ECL probes in sensors due to their chemical inertness, low cytotoxicity and ease of fabrication. The ECL mechanism of CQDs is suggested to involve the formation of excited-state CQDs ( $\left.\mathrm{R}^{*}\right)$ via electron-transfer annihilation of negatively charged $\left(\mathrm{R}^{-*}\right)$ and positively charged $\left(\mathrm{R}^{+*}\right)$ CQDs with the presence of peroxydisulfate $\left(\mathrm{S}_{2} \mathrm{O}_{8}{ }^{2-}\right)$, enhancing the ECL signal in the cathodic potential range. ${ }^{128} \mathrm{~A}$ multifunctional solid-state ECL sensing platform based on PEI capped N-doped CQDs (N-CQDs@PEI) as novel co-reactant of Ru(bpy) ${ }_{3}{ }^{+2}$ nanosheets (RuNSs) was designed by $\mathrm{Li}$ et al. (Figure 12B). ${ }^{129}$ The as-prepared N-CQDs@PEI displayed better catalytic effect to RuNSs than naked N-CQDs, due to the double enhancement contribution of N-CQDs and PEI to RuNSs. Simultaneously, reduced graphene oxide (rGO) was introduced to facilitate the electron transfer and amplify the ECL signal. The developed ECL sensor exhibited desired ECL emission, with about 69-fold enhancement of the ECL intensity in comparison with RuNSs/GCE and dopamine could be sensed over a wide linear range (0.01$50 \mu \mathrm{M}$ ) with a detection limit of $10 \mathrm{nM}$. GCE electrodes modified with glutamate oxidase as well as CQDs and luminol stabilized Ag NPs proved to be also effective ECL based sensors for the detection of different glutamate (Glu) enantiomers. ${ }^{130}$ Under optimized conditions and by applying a typical potential of $+0.6 \mathrm{~V}$, ECL increases linearly with increasing glutamate concentration, with a LOD of $1.6 \mu \mathrm{M}$. More lately, a CQDs based ECL sensor for the detection of miRNA-21 with a LOD of $10 \mathrm{aM}$ was provided. ${ }^{131}$ Such sensors were also applied for the ultrasensitive sensing of glutathione (GSH). ${ }^{123}$ on the sensor was prepared by electropolymerization of (11-pyrrolyl-1-ylundecyl) triethylammonium tetrafluoroborate (A2) with simultaneous embedding of hydrophilic CQDs on the surface of GCE. In the presence of $\mathrm{S}_{2} \mathrm{O}_{8}{ }^{2-}$ and using $\mathrm{Fe}(\mathrm{CN})_{6}{ }^{3-/ 4-}$ as redox couple, CQDs and $\mathrm{S}_{2} \mathrm{O}_{8}{ }^{2-}$ were converted into CQDs ${ }^{--}$ and $\mathrm{SO}_{4}{ }^{--}$. The $\mathrm{CDs}^{*}$ obtained through the recombination of electron- and hole-injected processes may be impeded due to the interference of GSH with $\mathrm{K}_{2} \mathrm{~S}_{2} \mathrm{O}_{8} .{ }^{132}$ This constructed sensor for GSH displayed a detection limit down to $54.3 \mathrm{nM}(\mathrm{S} / \mathrm{N}=3)$ and a wide linear range from 0.1 to $1.0 \mu \mathrm{M}$. By combining the intense ECL of CQDs and aptamer technology, Lu et al. developed an ECL aptamer sensor for measuring ATP. ${ }^{133}$ 
(A)

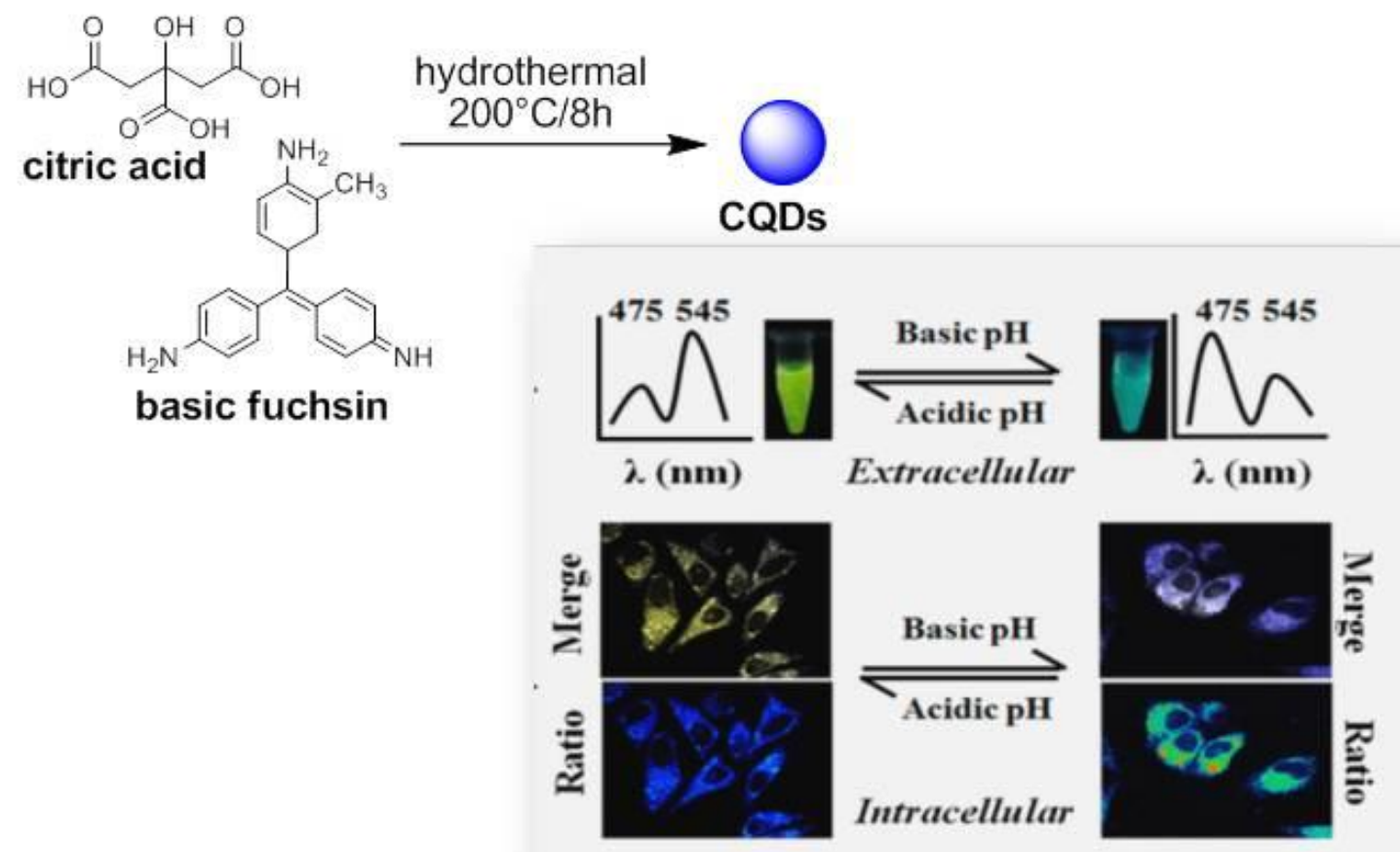

(B)

(a)

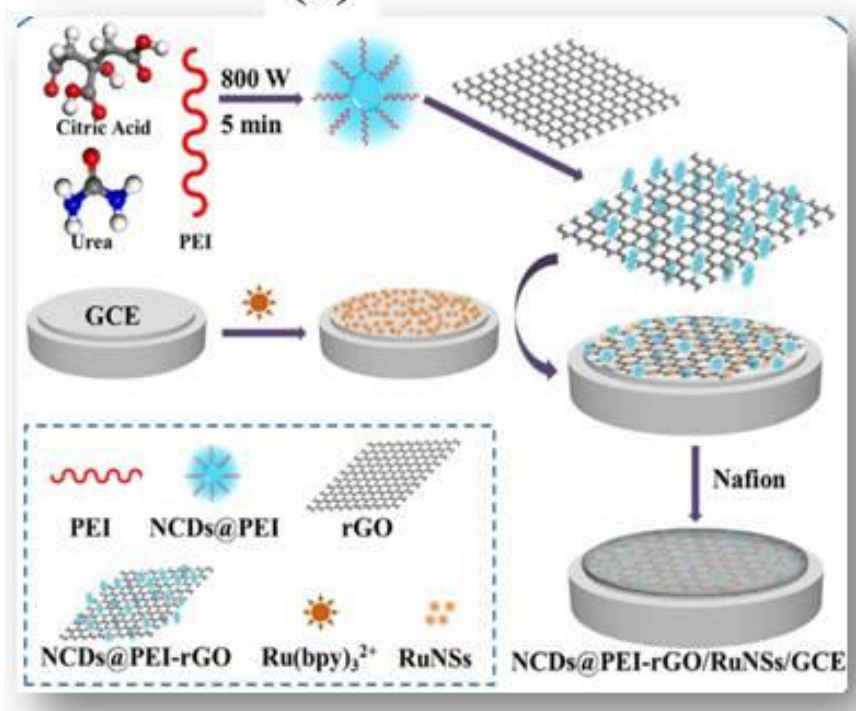

(b)

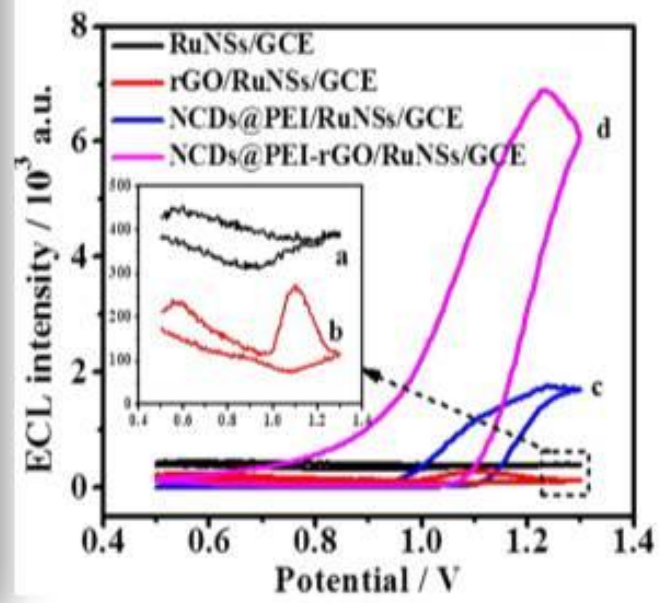

Figure 12: From fluorescence to electroluminescence based sensors: (A) Schematic diagram for the preparation of CQDs for intercellular $\mathrm{pH}$ sensing together with fluorescence spectrum of CQDs in different $\mathrm{pH}$ solutions under $380 \mathrm{~nm}$ excitation (reprinted with permission from Ref ${ }^{117}$ ); (B) (a) Schematic preparation of a solid-state ECL based sensor 
using N-doped CQDs@PEI-rGO/RuNSs/GCE, (b) ECL-potential curve of different electrodes in $50 \mathrm{mM}$ PBS ( $\mathrm{pH} 8.5$ ) (reprinted with permission from Ref. ${ }^{129}$ ).

Table 1: Florescence sensors using carbon-based quantum particles

\begin{tabular}{|c|c|c|c|c|c|c|}
\hline Carbon & $\begin{array}{l}\text { Synthesis } \\
\text { Method }\end{array}$ & Analyte & $\lambda_{\max } / \mathbf{n m}$ & LOD & Linear range & Ref. \\
\hline \multicolumn{7}{|l|}{ CDs } \\
\hline CDs & Hydrothermal & $\mathrm{Fe}(\mathrm{III})$ & $\begin{array}{l}600 \text { and } \\
680\end{array}$ & $1.9 \mathrm{nM}$ & $10-300 \mathrm{nM}$ & 103 \\
\hline CDs & Solvothermal & $\mathrm{Fe}(\mathrm{III})$ & $\begin{array}{l}426 \text { and } \\
488\end{array}$ & 9.6 and $7 \mu \mathrm{M}$ & $0-4000 \mu \mathrm{M}^{1}$ & 102 \\
\hline N/P co-doped CDs & Hydrothermal & $\mathrm{Fe}$ (III) & 408 & $0.33 \mu \mathrm{M}$ & $1-150 \mu \mathrm{M}$ & 134 \\
\hline CDs & $\begin{array}{l}\text { One-step } \\
\text { electrolysis }\end{array}$ & $\mathrm{Al}(\mathrm{III})$ & 510 & $0.05 \mu \mathrm{M}$ & $0.1-7.2 \mu \mathrm{M}$ & 104 \\
\hline $\begin{array}{l}\text { DNA-functionalized } \\
\text { CDs }\end{array}$ & Hydrothermal & $\mathrm{Hg}$ (II) & 451 & $1.02 \mathrm{nM}$ & $0-10 \mathrm{nM}$ & 108 \\
\hline CDs & Hydrothermal & $\begin{array}{l}\mathrm{Au}^{3+} \\
\mathrm{S}^{2-}\end{array}$ & 397 & $\begin{array}{c}63.1 \mathrm{nM} \\
92.4 \mathrm{nM}\end{array}$ & $\begin{array}{l}0-1 \mathrm{mM} \\
0-500 \mu \mathrm{M}\end{array}$ & 135 \\
\hline CDs & Hydrothermal & $\mathrm{Cr}^{6+}$ & 360 & $73 \mathrm{nM}$ & $2.5-50 \mu \mathrm{M}$ & 136 \\
\hline CDs & Hydrothermal & $\begin{array}{l}\text { Toxic } \\
\text { metal ions }\end{array}$ & $\begin{array}{l}410 \text { and } \\
450\end{array}$ & $10 \mu \mathrm{M}$ & $50-100 \mu \mathrm{M}$ & 137 \\
\hline CDs & $\begin{array}{l}\text { One-step } \\
\text { pyrolysis }\end{array}$ & $\begin{array}{l}\mathrm{Zn}^{2+} \\
\text { EDTA }\end{array}$ & 460 & $\begin{array}{l}\mathrm{Zn}^{2+} 51 \mu \mathrm{M} \\
\text { EDTA } 32 \mu \mathrm{M}\end{array}$ & $\begin{array}{l}\mathrm{Zn}^{2+} 2-15 \mu \mathrm{M} \\
\text { EDTA 2.5-25 } \mu \mathrm{M}\end{array}$ & 138 \\
\hline CDs & Hydrothermal & $\begin{array}{l}\mathrm{pH} \\
\mathrm{Cu}^{2+}\end{array}$ & $\begin{array}{l}630 \text { and } \\
590\end{array}$ & $40 \mu \mathrm{M}$ & $\begin{array}{l}\mathrm{Cu}^{2+} 0-200 \mu \mathrm{M} \\
\mathrm{pH}: 4-10\end{array}$ & 139 \\
\hline CDs & $\begin{array}{l}\text { Microwave } \\
\text { assisted } \\
\text { pyrolysis }\end{array}$ & $\mathrm{pH}$ & 450 & - & $\mathrm{pH}: 9-14$ & 140 \\
\hline CDs & Solvothermal & $\mathrm{pH}$ & 390 & - & $\mathrm{pH}: 3.3-7.5$ & 118 \\
\hline CDs & $\begin{array}{l}\text { Microwave } \\
\text { assisted } \\
\text { pyrolysis }\end{array}$ & $\mathrm{pH}$ & $531 / 413$ & - & $\mathrm{pH}: 4-8$ & 141 \\
\hline CDs & Solvothermal & Phosphate anions & 640 & $0.11 \mu \mathrm{M}$ & $0.5-6 \mu \mathrm{M}$ & 114 \\
\hline CDs & Hydrothermal & $\begin{array}{l}\text { Guanosine 3'- } \\
\text { diphosphate-5'- } \\
\text { diphosphate } \\
\text { (ppGpp) }\end{array}$ & 463 & $50 \mathrm{nM}$ & $0.5-15 \mu \mathrm{M}$ & 142 \\
\hline CDs & $\begin{array}{l}\text { One-step } \\
\text { refluxing }\end{array}$ & $\begin{array}{l}\text { caffeine } \\
\text { melamine } \\
\text { fenitrothion }\end{array}$ & $\begin{array}{l}400,495 \\
\text { and } 550\end{array}$ & $\begin{array}{l}\text { caffeine: } 1 \mu \mathrm{M} \\
\text { melamine: } 0.1 \mu \mathrm{M} \\
\text { menitrothion: } 1 \mu \mathrm{M}\end{array}$ & $\begin{array}{l}\text { Caffeine: } 1-75 \mu \mathrm{M} \\
\text { Melamine: } 0.1-20 \mu \mathrm{M} \\
\text { Fenitrothion: } 1-50 \mu \mathrm{M}\end{array}$ & 143 \\
\hline $\mathrm{Ag}^{+}$sensitive- $\mathrm{CDs}$ & Microwave & Biothiols & 430 & $\leq 10 \mu \mathrm{M}$ & - & 144 \\
\hline N-doped CDs & Reflux & $\begin{array}{l}\text { Catechol } \\
\text { Dopamine } \\
\text { L-dopa } \\
\text { Carbidopa } \\
\text { Epinephrine }\end{array}$ & 475 & $\begin{array}{l}30 \mathrm{nM} \\
61.2 \mathrm{nM} \\
15.3 \mathrm{nM} \\
86.2 \mathrm{nM} \\
21.5 \mathrm{nM}\end{array}$ & $\begin{array}{l}2.66-34 \mu \mathrm{M} \\
0.21-10 \mu \mathrm{M} \\
0.33-323 \mu \mathrm{M} \\
0.15-214 \mu \mathrm{M} \\
0.89-25.3 \mu \mathrm{M}\end{array}$ & 145 \\
\hline $\begin{array}{l}\text { CDs coupled } \\
\text { tyrosinase }\end{array}$ & Electrolysis & L-dopa & 485 & $90 \mathrm{nM}$ & $0.31 \mathrm{nM}-31 \mu \mathrm{M}$ & 146 \\
\hline $\begin{array}{l}\text { Carboxylic CDs } \\
\text { (citric and malic) }\end{array}$ & Pyrolysis & DNA & 530 & $\begin{array}{l}\text { citric : } 45.6 \mathrm{nM} \\
\text { malic : } 17.4 \mathrm{nM}\end{array}$ & $\begin{array}{l}\text { citric acid QD: } 0.4-400 \mathrm{nM} \\
\text { malic acid QD: } 0.04-400 \mathrm{nM}\end{array}$ & 126 \\
\hline $\begin{array}{l}\text { Hollow carbon } \\
\text { nanorods }\end{array}$ & pyrolysis & DNA & 460 & - & $1.14 \mathrm{nM}$ & 147 \\
\hline $\begin{array}{l}\text { Silane- } \\
\text { functionalized CDs }\end{array}$ & Sol-gel reaction & Nifedipine (NIF) & 440 & $0.076 \mu \mathrm{M}$ & $0.28-3.4 \mu \mathrm{M}$ & 148 \\
\hline $\mathrm{Au} @ \mathrm{CDs}$ & $\begin{array}{l}\text { Microwave } \\
\text { pyrolysis }\end{array}$ & Biothiols & 470 & $50 \mathrm{nM}$ & $0-30 \mu \mathrm{M}$ & 122 \\
\hline CDs & Oxidation & $\begin{array}{l}\text { Ochratoxin A } \\
\text { (OTA) }\end{array}$ & 433 & $13 \mathrm{pg} \mathrm{mL}^{-1}$ & $0-1 \mathrm{ng} \mathrm{mL}^{-1}$ & 103 \\
\hline CDs & $\begin{array}{l}\text { Hydrothermal } \\
\text { post- modif. } \\
\text { with APTES } \\
\end{array}$ & $\begin{array}{l}\text { trinitrotoluene } \\
\text { (TNT) }\end{array}$ & $450 \mathrm{~nm}$ & $4.4 \mathrm{nM}$ & $4.4 \mathrm{nM}-26.4 \mu \mathrm{M}$ & 121 \\
\hline CDs & $\begin{array}{l}\text { Hydrothermal } \\
\text { post-modif. } \\
\text { with } \\
\text { ehtylenediamine }\end{array}$ & $\begin{array}{l}\text { Trinitrotoluene } \\
\text { (intracellular) }\end{array}$ & $420 \mathrm{~nm}$ & $21 \mathrm{nM}$ & $88-267 n M$ & 149 \\
\hline CDs & Hydrothermal & Ampicillin & 427 & $16.5 \mu \mathrm{M}$ & $6.6-200 \mathrm{ppm}$ & 150 \\
\hline
\end{tabular}




\begin{tabular}{|l|l|l|l|l|l|l|}
\hline CDs & Hydrothermal & Tartrazine & 503 & $73 \mathrm{nM}$ & $0.25-32.50 \mu \mathrm{M}$ \\
\hline GO/CDs & Hydrothermal & Virginiamycin & 560 & $15.6 \mathrm{~nm} \mathrm{nM}$ & $5-800 \mathrm{nM}$ \\
\hline GQDs & $\begin{array}{l}\text { Chemical } \\
\text { oxidation }\end{array}$ & Fe (III) & 460 & $0.45 \mu \mathrm{M}$ & $0-60 \mu \mathrm{M}$ \\
\hline GQDs & Direct pyrolysis & Fe (III) & 460 & $7.6 \mathrm{nM}$ & $0.020-2 \mu \mathrm{M}$ \\
\hline GQDs & Pyrolysis & Glucose & 470 & $0.1 \mu \mathrm{M}$ & $9-300 \mu \mathrm{M}$ \\
\hline $\begin{array}{l}\text { Hemin- } \\
\text { functionalized } \\
\text { GQDs }\end{array}$ & & & & 152 \\
\hline Boronic acid GQDs & Hydrothermal & Glucose & 435 & $5.0 \mu \mathrm{M}$ & 105 \\
\hline $\begin{array}{l}\text { Boron-doped GQDs } \\
\text { High }\end{array}$ & $\begin{array}{l}\text { Glucose } \\
\text { temperature }\end{array}$ & Microwave & Melamine & 440 & $0.03 \mathrm{mM}$ & $1-10 \mathrm{mM}$ \\
\hline GQDs & Pyrolysis & Graphene oxide & 474 & $0.12 \mu \mathrm{M}$ & $0.1-10 \mathrm{mM}$ \\
\hline GQDs & Hydrothermal & Formaldehyde & 440 & $35 \mu \mathrm{g} \mathrm{L}$ & $0.15 \mu \mathrm{M}$ & $0.15-20 \mu \mathrm{M}$ \\
\hline N-doped GQDs & & & 154 \\
\hline
\end{tabular}

\subsubsection{Electrochemical sensors}

Excellent sensitivity, selectivity, simplicity, inexpensive instrumentation, fast response, environmental friendliness and portability are the main potential advantages of electrochemical-based sensing platform. Among the solid electrodes, carbon-based electrodes have attracted huge interest due to their remarkable properties such as high compatibility to be modified with various molecules, high conductivity, low back ground current, ease of preparation and inert chemical nature. Carbon-based electrodes have in addition a lower cost and wider anodic and cathodic potential window compared to platinum and gold electrodes (the potential window is limited in negative potentials regarding a platinum electrode due to the reduction of $\mathrm{H}^{+}$and in positive potentials due to the oxidation of a gold electrode's surface). This field has largely developed over the years through the implementation of CQDs, as labels as well as electrode material itself designed to have better rate of charge transport, selectivity, conductivity, stability and reproducibility. ${ }^{159}$ It has to be noted however, that in the case of electrochemical sensors, next to CQDs and GQDs, carbon nanoparticles (CNPs), not discussed here, have found wider use and the readers are referened to some of the papers on this topic. ${ }^{18,19,78,160-168}$ CQDs are now seen as crucial components in high performing electrochemical sensors as witnessed by the vast literature of the last 5 years summarized partially in Table 2. They can be used as a multivalent redox species using cyclic voltammetry and differential puls voltammetry (DPV) measurements as exemplified by Shinde and Pillai ${ }^{169}$ who showed a sequential, single-electron charging process of GQDs encapsulated in dodecylamine of about 2.2-3 nm in size. GQDs have shown also their use in the construction of a single-electron transistors (SET), a new type of switching device that uses controlled electron tunneling to current amplification. ${ }^{170}$ This all made CQDs and GQDs to be recognized as optimal electrode materials for electroanalysis.

\section{Metal ion sensing}

Currently, the examples of metal ion sensing using CQDs are limited to the use of N-CQDs mixed with graphene oxide (GO) used for the simultaneous determination of $\mathrm{Cd}^{2+}$ and $\mathrm{Pb}^{2+}$ using anodic stripping voltammetry (Figure 13A). ${ }^{171}$ In the hybrid, both N-CQDs and GO have large electroactive surface areas and abundant oxygen-containing functional groups, which supply active sites for the efficient adsorption of heavy metal ions by electrostatic interaction to improve the sensor sensitivity. The N-CQDs is homogeneously distributed on the surface of GO and plays an important role in re-oxidizing metals into ions for stripping 
analysis. Based on these advantages, the electrochemical $\mathrm{Cd}^{2+}$ and $\mathrm{Pb}^{2+}$ sensor using a NCQDs-GO hybrid modified glassy carbon electrode exhibited superior analysis performance with low detection limits $\left(7.45 \mu \mathrm{g} \mathrm{L}^{-1}\right.$ for $\mathrm{Cd}^{2+}$ and $1.17 \mu \mathrm{g} \mathrm{L}^{-1}$ for $\mathrm{Pb}^{2+}$, respectively), good reproducibility and selectivity.

\section{Chemical sensors}

Since more than 50 years has electrochemistry has been used as a powerful analytical technique for monitoring electroactive species in living organisms such dopamine. Dopamine, produced in adrenal glands and several areas of the brain, and is the most abundant of the catecholamines involved in brain-body integration and crucially important in humans. Given the wide range of physiological and pathophysiological implications, the development of a sensor for precise and selective measurement of dopamine (and/or its metabolites) at the low levels characteristic of living system $(26-40 \mathrm{nM})^{172}$ can make a great contribution to disease diagnosis. Drop casting of carbon-based quantum particles, obtained by a green variant of Hummers' method, onto glassy carbon electrode (GCE) allows to construct a sensor for dopamine with a detection limit of $2.7 \mu \mathrm{M} .{ }^{173}$ The sensitivity could be improved by employing $\beta$-cyclodextrin (CD) reaching a LoD of $140 \mathrm{nM} .{ }^{174} \mathrm{~N}$-doped CQDs, formed using diethanolamine as nitrogen and carbon source, coated onto GCE resulted finally in a dopamine sensor with a limit of detection of $1.2 \mathrm{nM}^{175}$ for use in urine and blood serum but also for eventual characteristic of living systems.

$\mathrm{Li}$ et $a l .{ }^{176}$ constructed a nanohybrid of $\mathrm{Co}_{3} \mathrm{O}_{4}$-histidine-functionalised GQDs for the modification of GCE, which demonstrated high electrocatalytic activity toward hydroquinones with a detection limit of $820 \mathrm{nM}(\mathrm{S} / \mathrm{N}=3)$. Tan et al. adopted a similar strategy to fabricate a high performance MIP/GQDs-based sensor, for bisphenol A. ${ }^{177}$ In other report by Prasad et al., MIP/GQDs based sensors were employed for the selective sensing of ifosfamide, an anticancer drug. ${ }^{178}$

Due to the significance of hydrogen peroxide $\left(\mathrm{H}_{2} \mathrm{O}_{2}\right)$ in biological systems and its practical applications, the development of efficient electrochemical $\mathrm{H}_{2} \mathrm{O}_{2}$ sensors holds a special attraction for researchers. ${ }^{179}$ Such sensors provide tremendous potential for real-time tracking of $\mathrm{H}_{2} \mathrm{O}_{2}$ in living systems. Indeed, $\mathrm{H}_{2} \mathrm{O}_{2}$ is an indicator of oxidative stress, closely related to physiological and pathological events such as aging, cancer, ischemia/reperfusion injury, traumatic brain injury, impaired learning and memory function etc. The $\mathrm{H}_{2} \mathrm{O}_{2}$ releasing level under normal physiological conditions is in the concentration range of $10-100 \mathrm{nM}$, with increased concentration of up to $10 \mu \mathrm{M}$ in stressed state. The synergetic effect between nanomaterials and electrochemical $\mathrm{H}_{2} \mathrm{O}_{2}$ determination is highlighted by the fact that electrocatalytic $\mathrm{H}_{2} \mathrm{O}_{2}$ determinations provide superior sensing performance over nonelectrocatalytic ones in various aspects. The use of CQDs have found interest in this field. ${ }^{180-}$ 183 Muthusankar et al. constructed a N-CQD/Cu $\mathrm{Cu}_{2} \mathrm{O}$ nanocomposite electrode through the electrodeposition of N-CQDs on the surface of GCE and decoration with $\mathrm{Cu}_{2} \mathrm{O}$ microstructures for the sensing of aspirin with a LOD of $2 \mu \mathrm{M} .{ }^{180} \mathrm{Su}$ et al. applied for example $\mathrm{NH}_{2}$-modified CQDs for the immobilization of horseradish peroxidase (HRP) and the detection of hydrogen peroxide between 5 - $590 \mathrm{nM}$ with a detection limit of (Figure 
13C). ${ }^{182}$ Zhang used ther high peroxidase-like property of carboxylic acid group enriched GQDs for enzyme free hydrogen peroxide sensing. ${ }^{183}$

Chemicals sensing of neurotransmitters such as serotonin was achieved with GQDshexagonal boron nitride nanostructures, known under the name of white graphene due to its remarkable features such as excellent conductivity, high stability and large specific surface area, which successfully enhanced electrochemical properties of GQDs. Serotonin imprinted polymer films were formed on the surface of the modified GCE via electrochemical polymerization, allowing serotonin sensing with a sensitivity down to $0.2 \mathrm{nM}^{184}$

The decoration of carbon nanostructures with $\mathrm{Ag} \mathrm{NPs}$, $\mathrm{Au}$ NPs $\mathrm{Fe}_{3} \mathrm{O}_{4} \mathrm{NPs}$, etc. allows in many cases to amplify the electrical conductivity of CQDs. Guo et al. used silver nanoparticles/CQDs/reduced graphene oxide nanocomposite for the sensing of doxorubicin with a LOD of $2 \mathrm{nM} .^{185}$ The combination of a high conductive carbon nanostructure like MWCNTs with GQDs and $\mathrm{Fe}_{3} \mathrm{O}_{4}$ NPs resulted in the formation of a three dimensioned porous GQDs/ $/ \mathrm{Fe}_{3} \mathrm{O}_{4} / \mathrm{MWCNT}$ nanohybrid, ${ }^{186}$ exhiting a higher electrocatalytic activity towards progesterone with a LOD of $2.18 \mathrm{nM}$ and a sensitivity of $16.84 \mu \mathrm{A} \mu \mathrm{M}^{-1} .{ }^{187}$ In the same year, Arvand et al. fabricated a graphene quantum dots and poly (sulfosalicylic acid) co-modified electrode for the simultaneous detection of estradiol and progesterone, with LOD of $0.23 \mathrm{nM}$ and $0.31 \mathrm{nM}$, respectively. ${ }^{188}$

\section{Biochemical sensing}

When it comes to electrochemical biosensors using CQDS, the literature is still rather empty. $\mathrm{n}$ electrochemical immuno-sandwich sensor based on GQDs deposited on the surface of carbon screen-printed electrode (SPE) to detect the toxic fraction Aah50 of the Androctonus australis hector (Aah) scorpion venom was reported. ${ }^{189}$ Canevari et al. coated SWNT/CQDs on carbon electrodes for the sensing of $17 \alpha$-ethynylestradiol, reflecting an increase of the active area of the hybrid material, promoted by the CQDs. However, the best activity was observed upon immobilization of laccase, suggesting that the nanocrystalline CQDs improve the electronic transport between the substrate, SWNT and the copper ions in the enzyme active sites. ${ }^{190}$ 


\section{(A)}
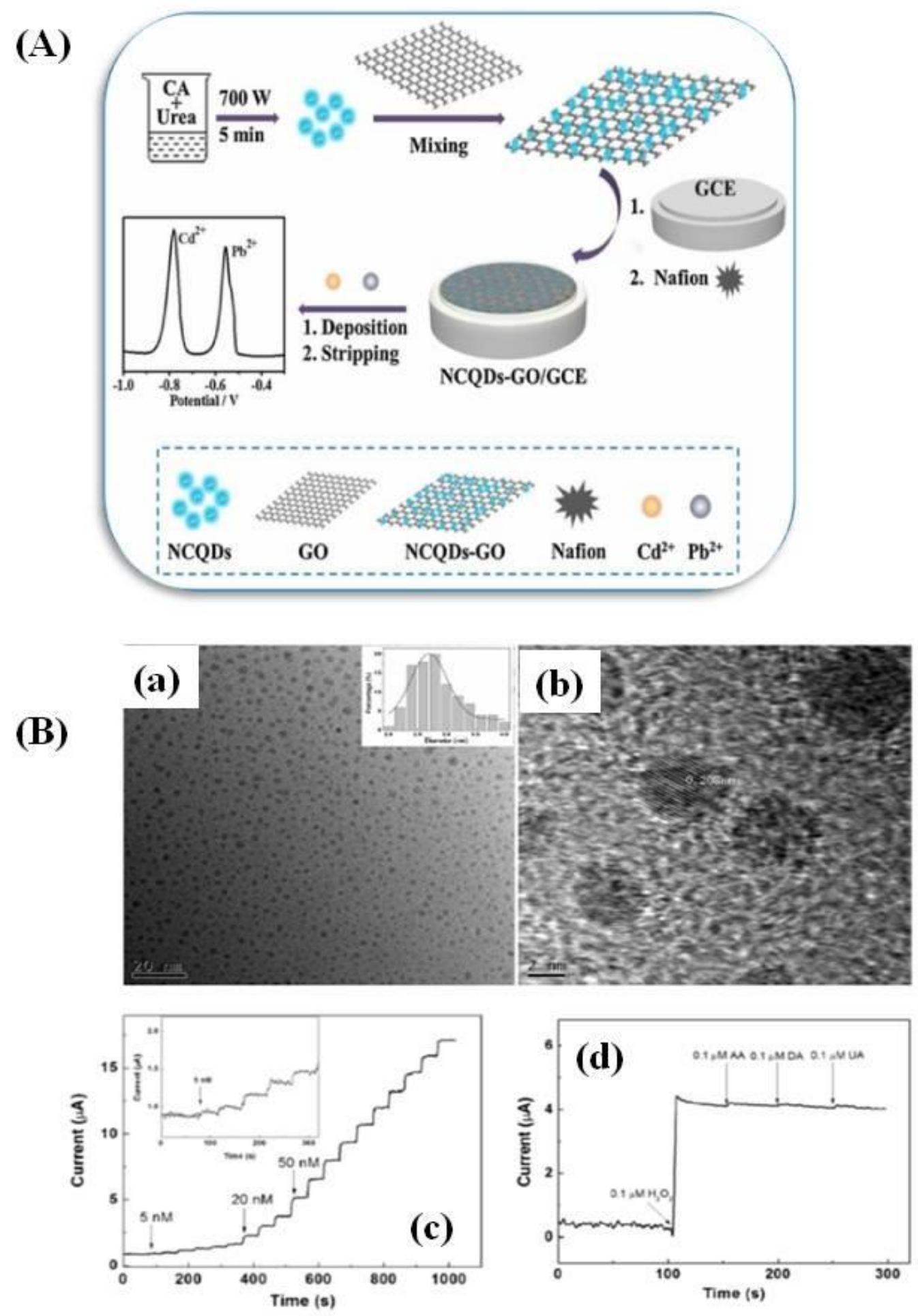

Figure 13. Electrochemical sensors: (A) N-doped CQDs-GO/GCE sensor for the sensing of metal ions (reprinted with permission from Ref. ${ }^{96,191}$ ); (B) Use of glassy carbon electrodes modified with CQDs modified with HRP for selective sensing of $\mathrm{H}_{2} \mathrm{O}_{2}$ : (a) TEM and (b) HR-TEM image of amino-functionalized CQDs, (c) Chronoamperometric response at $-0.3 \mathrm{~V}$ to $\mathrm{H}_{2} \mathrm{O}_{2}$ additions, (d) Amperometric response to $\mathrm{H}_{2} \mathrm{O}_{2}(0.1 \mu \mathrm{M})$ in the presence of ascorbic 
acid (AA, $0.1 \mu \mathrm{M}$ ), dopamine (DA, $0.1 \mu \mathrm{M}$ of) and uric acid (UA, $0.1 \mu \mathrm{M}$ of) (reprinted with permission from Ref. ${ }^{182}$ ).

Table 2 clearly states that to improve the electrochemical activity, carbon quantum particles based nanocomposits should to be used. It is mainly the synergy of the physcio-chemcial properties of materials which can be put forward as the main factor for the improved electrochemical performance, something well known for other cartbon based materials. ${ }^{192,}$ 193 Taking the example of metal ion sensing using N-N-CQDs and GO, in the hybrid, NCQDs and GO have large electroactive surface areas and abundant oxygen-containing functional groups, which supply active sites for the efficient adsorption of heavy metal ions by electrostatic interaction to improve the sensor sensitivity.

Table 2. Recent electroanalytical applications of carbon-based quantum particles modified electrodes

\begin{tabular}{|c|c|c|c|c|c|}
\hline Electrode & Modifier & Analyte & Linear range & LOD & Ref \\
\hline \multicolumn{6}{|l|}{ CDs } \\
\hline GCE & CDs & Glucose & $\begin{array}{l}0.5-40 \mu \mathrm{M} \\
50-600 \mu \mathrm{M}\end{array}$ & $0.09 \mu \mathrm{M}$ & 194 \\
\hline GCE & $\begin{array}{l}\text { Amino-CDs immobilized with } \\
\text { horseradish peroxidase }\end{array}$ & $\mathrm{H}_{2} \mathrm{O}_{2}$ & $5-500 \mathrm{nM}$ & $1.8 \mathrm{nM}$ & 182 \\
\hline GCE & AgNPs/CDs & $\mathrm{H}_{2} \mathrm{O}_{2}$ & $0.2-27.0 \mu \mathrm{M}$ & $80 \mathrm{nM}$ & 195 \\
\hline GCE & Poly(alizarin yellow R)/CDs & L-cysteine & $\begin{array}{l}0.3-3.6 \mu \mathrm{M} \\
3.9-7.2 \mu \mathrm{M}\end{array}$ & $90 \mathrm{nM}$ & 196 \\
\hline GCE & CDs & Dopamine & $1-7 \mu \mathrm{M}$ & $0.099 \mu \mathrm{M}$ & 197 \\
\hline CFM & $\mathrm{rGO} / \mathrm{CDs}$ & Dopamine & $0.1-100 \mu \mathrm{M}$ & $0.02 \mu \mathrm{M}$ & 198 \\
\hline GCE & Graphene/CDs & $\begin{array}{l}\text { Uric acid } \\
\text { Ascorbic acid }\end{array}$ & - & - & 76 \\
\hline GCE & CTAB/chitosan /CDs & 2,4-dichlorophenol & $0.04-8.0 \mu \mathrm{M}$ & $0.01 \mu \mathrm{M}$ & 199 \\
\hline GCE & $\mathrm{rGO} / \mathrm{CDs}$ & Rutoside & $3 \mathrm{nM}$ & $0.01-6.5 \mu \mathrm{M}$ & 200 \\
\hline $\begin{array}{l}\text { Carbon paste } \\
\text { electrode }\end{array}$ & CDs@ $\mathrm{CuFe}_{2} \mathrm{O}_{4}$ nanocomposite & $\begin{array}{l}\text { Rifampicin (RIF) } \\
\text { Isoniazid (INZ) }\end{array}$ & $\begin{array}{l}\text { RIF } 0.07-8.0 \mu \mathrm{M} \text { INZ } \\
0.1-14.0 \mu \mathrm{M}\end{array}$ & $\begin{array}{l}\text { RIF } 22 \mathrm{nM} \\
\text { INZ } 41 \mathrm{nM}\end{array}$ & 201 \\
\hline ITO glass & CDs-modified chitosan & Vitamin $\mathrm{D}_{2}$ & $10-50 \mathrm{ng} \mathrm{mL}^{-1}$ & $1.35 \mathrm{ng} \mathrm{mL}^{-1}$ & 202 \\
\hline GCE & $\begin{array}{l}\text { Flower-like NiAl } \\
\text { double hydroxides } \\
\text { with CDs }\end{array}$ & Acetylcholine & $5-6885 \mu \mathrm{M}$ & $1.7 \mu \mathrm{M}$ & 203 \\
\hline GCE & CDs & Etoposide & $0.06-100 \mu \mathrm{M}$ & $5 \mathrm{nM}$ & 204 \\
\hline GCE & Pd-Au@CDs & Colitoxin DNA & $\begin{array}{l}5.0 \times 10^{-16} \text { to } 1.0 \times 10^{-10} \\
\mathrm{M}\end{array}$ & $1.82 \times 10^{-17} \mathrm{M}$ & 205 \\
\hline GCE & $\begin{array}{l}\text { Laccase enzyme supported on } \\
\text { SWNT/CDs } \\
\text { decorated with CDs }\end{array}$ & Ethynylestradiol & $0.05-7 \mu \mathrm{M}$ & $4.0 \mathrm{nM}$ & 190 \\
\hline MIP & Chitosan/ AuNPs/CDs & Patulin & $0.01-1.0 \mathrm{nM}$ & $0.00757 \mathrm{nM}$ & 206 \\
\hline GCE & CDs & $\begin{array}{l}\text { Curcumin } \\
\text { metanil yellow }\end{array}$ & $\begin{array}{l}0.4-200 \mu \mathrm{M} \\
0.4-200 \mu \mathrm{M} \\
\end{array}$ & $\begin{array}{l}0.1 \mu \mathrm{M} \\
0.03 \mu \mathrm{M} \\
\end{array}$ & 207 \\
\hline \multicolumn{6}{|l|}{ GQDs } \\
\hline GCE & $\begin{array}{l}\text { Decorated N-doped GQ } \\
\text { Ds@N-doped carbon hollow } \\
\text { nanospheres }\end{array}$ & Biomarker $\mathrm{H}_{2} \mathrm{O}_{2}$ for cancer detection & $0-1.4 \mathrm{mM}$ & $20 \mathrm{nM}$ & 80 \\
\hline GCE & N-doped GQDs & $\mathrm{H}_{2} \mathrm{O}_{2}$ & $0.25-13327 \mu \mathrm{M}$ & $0.12 \mu \mathrm{M}$ & 208 \\
\hline GCE & $\begin{array}{l}\text { Pd Nanoparticles decorated N- } \\
\text { doped GQD@N-doped carbon } \\
\text { hollow nanospheres }\end{array}$ & $\mathrm{H}_{2} \mathrm{O}_{2}$ & - & $20 \mathrm{nM}$ & 80 \\
\hline GCE & $\begin{array}{l}\text { Gold nanoparticles on nitrogen- } \\
\text { doped GQDs }\end{array}$ & $\mathrm{H}_{2} \mathrm{O}_{2}$ & $0.25-13327 \mu \mathrm{M}$ & $0.12 \mu \mathrm{M}$ & 208 \\
\hline
\end{tabular}




\begin{tabular}{|c|c|c|c|c|c|}
\hline $\mathrm{CPE}$ & $\begin{array}{l}\text { CoNiAl-layered double- } \\
\text { hydroxide } \\
\text { nanocomposite/GQDs }\end{array}$ & Glucose & $0.01-14.0 \mathrm{mM}$ & $6 \mu \mathrm{M}$ & 209 \\
\hline GCE & $\mathrm{NiO} / \mathrm{GQDs}$ & Clozapine & $3 \mathrm{nM}-1 \mu \mathrm{M}$ & $0.55 \mathrm{nM}$ & 210 \\
\hline $\mathrm{CCE}$ & $\mathrm{RuCl}_{3} / \mathrm{GQDs}$ & L-tyrosine & $1-937 \mu \mathrm{M}$ & $0.23 \mu \mathrm{M}$ & 211 \\
\hline GCE & GQD- $\beta$-Cyclodextrin & L-Cysteine & $0.01-2 \mathrm{mM}$ & N.A & 212 \\
\hline GCE & $\alpha \mathrm{CD}-\mathrm{AgNPs}$ & Aflatoxin M1 & $0.015-25 \mathrm{mM}$ & $2 \mu \mathrm{M}$ & 213 \\
\hline GCE & N-GQDs & 2,4,6-Trinitrotoluene & $1-400 \mathrm{ppb}$ & $0.2 \mathrm{ppb}$ & 186 \\
\hline GCE & GQDs & Doxorubicin & $0.018-3.600 \mu \mathrm{M}$ & $0.016 \mu \mathrm{M}$ & 214 \\
\hline GCE & GQDs & $\begin{array}{l}\text { Hydroquinone } \\
\text { catechol }\end{array}$ & $\begin{array}{l}4.0-600 \mu \mathrm{M} \\
6.0-400 \mu \mathrm{M}\end{array}$ & $\begin{array}{l}0.40 \mu \mathrm{M} \\
0.75 \mu \mathrm{M}\end{array}$ & 215 \\
\hline GCE & GQDs & Epinephrine & $1-120 \mu \mathrm{M}$ & $83 \mathrm{nM}$ & 216 \\
\hline CSP & GQDs & $\begin{array}{l}\text { Androctonus australis } \\
\text { hector }\end{array}$ & $40-700 \mathrm{pg} \mathrm{mL}^{-1}$ & $0.55 \mathrm{pg} \mathrm{mL}^{-1}$ & 189 \\
\hline GCE & $\begin{array}{l}\text { GQDs-ionic liquid-nafion } \\
\text { nanomatrix }\end{array}$ & Carcinoembryonic antigen & $\begin{array}{l}0.5 \text { fg } \mathrm{mL}^{-1}-0.5 \mathrm{ng} \\
\mathrm{mL}^{-1}\end{array}$ & $0.34 \mathrm{fg} \mathrm{mL}^{-1}$ & 217 \\
\hline CSP & GQDs-MIP & Anti-cancerous ifosfamide & - & $11 \mathrm{ng} \mathrm{L}^{-1}$ & 218 \\
\hline GCE & Au NPs & $\begin{array}{l}\mathrm{Hg}^{2+} \\
\mathrm{Cu}^{2+}\end{array}$ & $0.02-1.5 \mathrm{nM}$ & $\begin{array}{l}0.02 \mathrm{nM} \\
0.05 \mathrm{nM}\end{array}$ & 219 \\
\hline $\begin{array}{l}\text { Pencil } \\
\text { graphite } \\
\text { electrode }\end{array}$ & GQDs & $\mathrm{Cu}^{2+}$ & $50 \mathrm{pM}-4 \mathrm{nM}$ & $12 \mathrm{pM}$ & 220 \\
\hline GCE & ERGQDs & Uranium & $23.4-345.8 \mathrm{ppb}$ & $2.0 \mathrm{ppb}$ & 221 \\
\hline GCE & Riboflavin/GQDs & Persulfate & $1.0 \mu \mathrm{M}-1 \mathrm{mM}$ & $0.2 \mu \mathrm{M}$ & 222 \\
\hline
\end{tabular}

CPE: Carbon paste electrode; CCE: carbon composite electode, ERGQD: Electrochemically reduced GQDs; N-GQDs: Nitrogen-doped graphene quantum dots.

\subsection{Recent Biomedical applications}

While the use of sensors is increasing day-by-day in the real world to improve the quality of life by providing information on medical diagnostics for healthcare, much work has in addition been exploited using the same nanomaterials in different biomedical applications such as bioimaging, drug delivery until the ablation of pathogens and the inhibition of protein fibrillation. Indeed, most particles used for sensing can be also used for biomedical applications, once their cytotoxicity has been validated. Like other nanoparticles, carbonbased quantum nanoparticles can modulate cell fate, induce or prevent mutations, initiate cellcell communication, and modulate cell structure in a manner dictated largely by phenomena at the nano-bio interface. ${ }^{223}$

\subsubsection{Bioimaging}

More and more, CQDs and GQDs are seen as excellent alternatives for bioimaging applications, replacing traditional quantum dots such as CdTe, CdSe and related core-shell nanoparticles in vitro and in vivo optical imaging experiments. As these quantum dots contain toxic heavy metals, their application has raised concerns. CQDs are general nontoxic and exhibit excellent photo-luminescent properties. Their extremely small size favors their uptake by cells via endocytosis. The pioneering work on CQDs for bioimaging was that of Sun et al. who demonstrated the potential of PEGylated CQDs for two-photon luminescence microcopy using human breast cancer MCF-7 cells. ${ }^{70}$ When it comes to bioimaging of tissue, the most important obstacle to overcome is that the imaging target (i.e. tissue, cells, etc.) absorbs almost all visible wavelengths of light. Higher energy electromagnetic radiation such ultraviolet light can harm the imaging target in several ways. Considering all of these hurdles together, only a narrow range of wavelengths can be used to study biological systems. This 
range of wavelengths lies between $650-900 \mathrm{~nm}$, the optical therapeutic window. The depth of tissue penetration varies on the wavelength used. Blue light $(450 \mathrm{~nm})$ will penetrate to a depth of about $0.1 \mathrm{~mm}$. Yellow light $(600 \mathrm{~nm})$ penetrates a depth of about $2.0 \mathrm{~mm}$ and red light $(680 \mathrm{~nm})$ about $3.0 \mathrm{~mm}$. Tao et al. performed in vivo fluorescence imaging of CQDsinjected in mice at different excitation wavelengths. The best fluorescence contrast was observed at an excitation of $595 \mathrm{~nm}^{224}$

Some of us recently performed cellular bioimaging experiments using different functionalized CQDs on HuV7 cell lines to investigate the uptake mechanism of the particles (Figure 14A). The green fluorescence observed is attributed to CQDs, homogeneously distributed in the cytoplasm in the case of CQDs-3 (hydrothermal synthesis using citric acid and ethylene amine), which confirms the internalization of CQDs inside the cells. The reduction of green fluorescence observed in the cytoplasm after $1 \mathrm{~h}$ incubation at $4{ }^{\circ} \mathrm{C}$ suggests that the active internalization mechanism may be partially blocked and a small portion of CQDs was internalized by passive penetration.

(A)
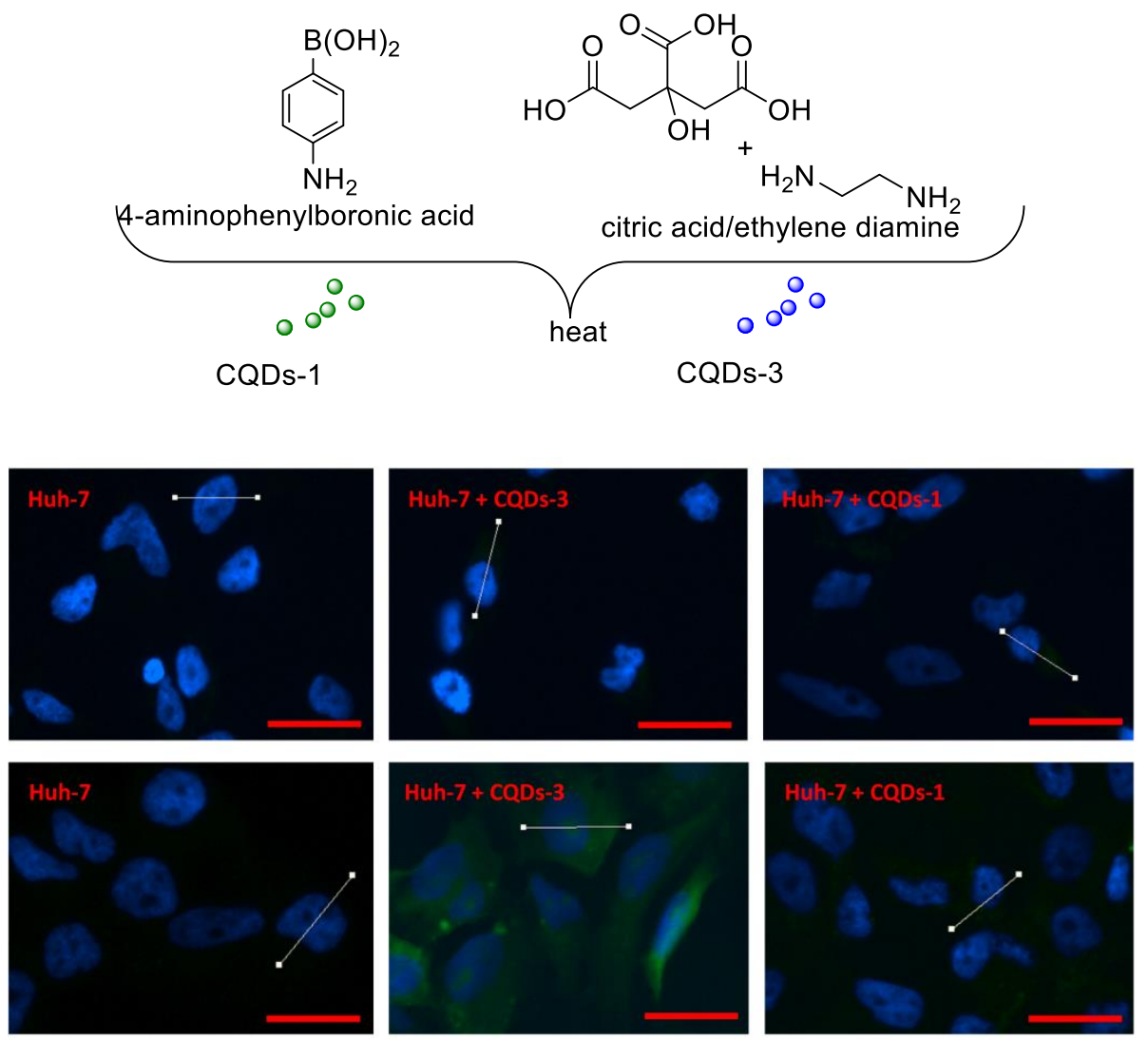

Figure 14: Fluorescence microscopy images of Huh-7 cells treated with $100 \mu \mathrm{g} \mathrm{mL}^{-1}$ of CQDs for $1 \mathrm{~h}$ at $4{ }^{\circ} \mathrm{C}$ (upper) and $37{ }^{\circ} \mathrm{C}$ (lower). The blue signal corresponds to the nuclei stained with Hoechst 33342, while the green signal may be attributed to CQDs. Scale bars = $50 \mu \mathrm{m}$ (unpublished results Szunerits et al.) 


\subsubsection{Drug delivery systems}

Tremendous efforts are devoted to the development of efficient drug delivery systems to improve chemotherapy and to depress toxic side effects. Graphene has demonstrated excellent drug carrier properties. However, to reach the nucleus, the nanomaterial has to pass the intracellular biological barrier. While the nuclear membrane has pores of $20-70 \mathrm{~nm}$ in diameter, it is almost impermeable to most probes, requiring the development of nucleartarget carries ${ }^{225}$. The excellent water dispersability of GQDs together with good drug loading capacity and small size, makes them idea candidates. Motivated by the capability to increase the chemotherapeutic efficiency of anticancer drugs, Zhou et al. demonstrated that GQDs with small lateral size show good intercalating ability toward DNA molecules.226 Furthermore, GQDs can efficiently accelerate the nuclear accumulation of DNA intercalating drugs, which enhances markedly the DNA cleavage activity and drug cytotoxicity. ${ }^{227}$ Several cancer drug systems have been reported ${ }^{13}$ with doxorubicin (DOX) being the most investigated anticancer drug as it allows drug uptake to be tracked via fluorescence microscopy due to the inherent fluorescence nature of DOX.

To increase local therapeutic effects and to minimize side effects of non-cancerous tissue targeted drug delivery is required. GQDs formed by the solvothermal method with an average size of $5 \mathrm{~nm}$ and labelled with TAT, a peptide that is able to bind $\alpha$ and $\beta$-importin, to was proposed. ${ }^{228}$ The peptide was grafted on the GQDs surface using a PEG polymer spacer and DOX was loaded to the system by $\pi-\pi$ interactions (Figure 15A). This nanostructure greatly contributed to nuclear targeting and resulted in a significant enhanced intra-nuclear DOX accumulation. For a DOX concentration of $10 \mu \mathrm{g} \mathrm{mL} \mathrm{m}^{-1}, 62.5 \%$ toxicity was reached with these nanocomposites, while the non-labeled GQDs and the drug alone showed toxicity of 17.1 and $19.9 \%$, respectively. ${ }^{228}$ 

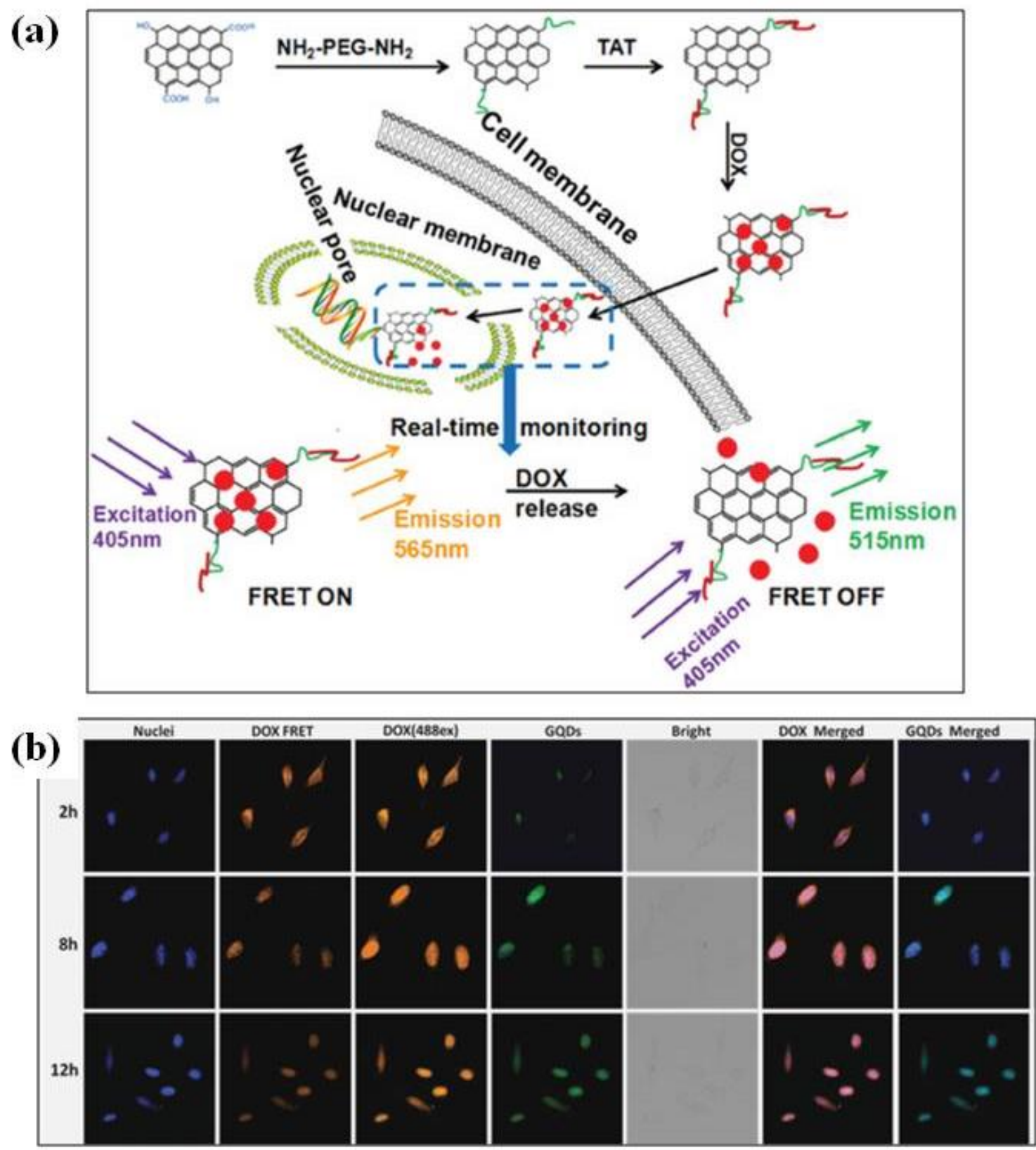

Figure 15: Drug delivery: (A) Cell nucleus-targeted drug deliver using GQDs which serves simultaneously as FRET sensor to follow uptake, (b) Confocal fluorescence images describing the changes in FRET signals in HeLa cells treated with DOX-GQDs-PEG/TAT at 2, 8 and $12 \mathrm{~h}$. HeLa cells were excited at $405 \mathrm{~nm}$. GQDs channel (485-515 nm), DOX FRET channel (565-595 nm) (reprint with permission from Ref. ${ }^{228}$ ).

\subsubsection{Antibacterial and antiviral properties of carbon nanostructures}

Carbon-based nanomaterials are offer promising potential to engage with biological molecules and have been found to possess bactericidal properties toward pathogenic microorganisms. The mechanism by which these materials inactivate bacteria is complex and depends on the intrinsic properties the materials such as composition, surface modification etc. Lately, CQDs and GQDs have to be added to the list of nanomaterials used for treatment of pathogens ${ }^{229-238}$ and viruses ${ }^{40,239}$ The intrinsic antibacterial activity of these nanomaterials are mainly dependent on the synthetic rout employed and the carbon source used. ${ }^{233}$ GQDs, 
prepared from GO with a planar geometry, lack antibacterial properties, while small-lateral sized GQDs can penetrate the plasma membrane and cause oxidative stress and rupture of the membrane. GQDs have intrinsic peroxidase-like activity, which can decrease the viability of Escherichia coli (E. coli) and Staphylococcus aureus (S. aureus) upon the addition of $1 \mathrm{mM}$ $\mathrm{H}_{2} \mathrm{O}_{2}{ }^{234}$ A photodynamic effect was reported by Trajkovic and coworkers upon irradiation at $470 \mathrm{~nm}$ via a two-photon excitation process. ${ }^{235}$ Two-photon excitation is of interest due to its effective penetration ability into biological tissue with a minimal damage to adjacent healthy tissue and reduced photo-bleaching. Recently, nitrogen-doped GQDs carrying amine functions (NGQD $\mathrm{NH}_{\mathrm{N} 2}$ ) were reported to have even better ability to produce photo-induced reactive oxygen species (ROS) than undoped GQD, resulting in improved antibacterial ability. ${ }^{236}$

Based on a series of biochemical experiments, it was established that uncharged CQDs have no effect on bacterial growth, while negatively and positively charged CQDs can induce bacteria apoptosis and bacteria death. ${ }^{229}$ Highly positively charged CQDs ( $\zeta$-potential ca. +45 $\mathrm{mV}$ ), synthesized by direct pyrolysis of spermidine powder through a simple dry heating treatment forming $\mathrm{CQD}_{\text {Spds }}$ (Figure 16A), proved to be efficient antibacterial agents against E. coli, S. aureus, Pseudomonas aeruginosa, Salmonella enterica and Serovar Enteritidis. Most importantly, CQD ${ }_{\text {Spds }}$ are active against methicillin-resistant $S$. aureus. The minimal inhibitory concentration (MIC) of CQD $\mathrm{Cpds}_{\text {is }} \sim 2500$-fold lower than that of spermidine alone, demonstrating their strong antibacterial capabilities due to the highly positive charge, which causes severe disruption of the bacterial membrane. CQD ${ }_{\text {Spds }}$ have demonstrated additional benefits for clinical applications in treating eye-related bacterial infections. It was shown that CQD ${ }_{\text {Spds }}$ were efficient for topical ocular administration where they induce the opening of the tight junction of corneal epithelial cells, thereby leading to great antibacterial treatment of $S$. aureus-induced bacterial keratitis in rabbits (Figure 16A). ${ }^{229}$ Amine-functionalized CQDs $\left(\mathrm{CQD}_{\mathrm{NH} 2}\right)$ have also proven to be of interest as antibacterial agents. $\mathrm{CQD}_{\mathrm{NH} 2}$ modified with lauryl betaine allowed for selective bacteria labeling and killing of Gam-positive bacteria. ${ }^{232}$ Some of us showed lately that $\mathrm{CQD}_{\mathrm{NH} 2}$ can be used as carrier of antibiotics such as ampicillin. ${ }^{237}$ Compared to free ampicillin, the ampicillin modified $\mathrm{CQD}_{\mathrm{NH} 2}$ have a higher stability and displayed effective E. coli killing as a result of combined antibacterial and photodynamic effect of $\mathrm{CQD}_{\mathrm{NH} 2}$ (Figure 16B). ${ }^{237}$ In this line, Sun and coworkers reported CQDs modified with ethylenediamine (EDA) as an efficient antibacterial agent under photoillumination. ${ }^{238}$ 
(A)
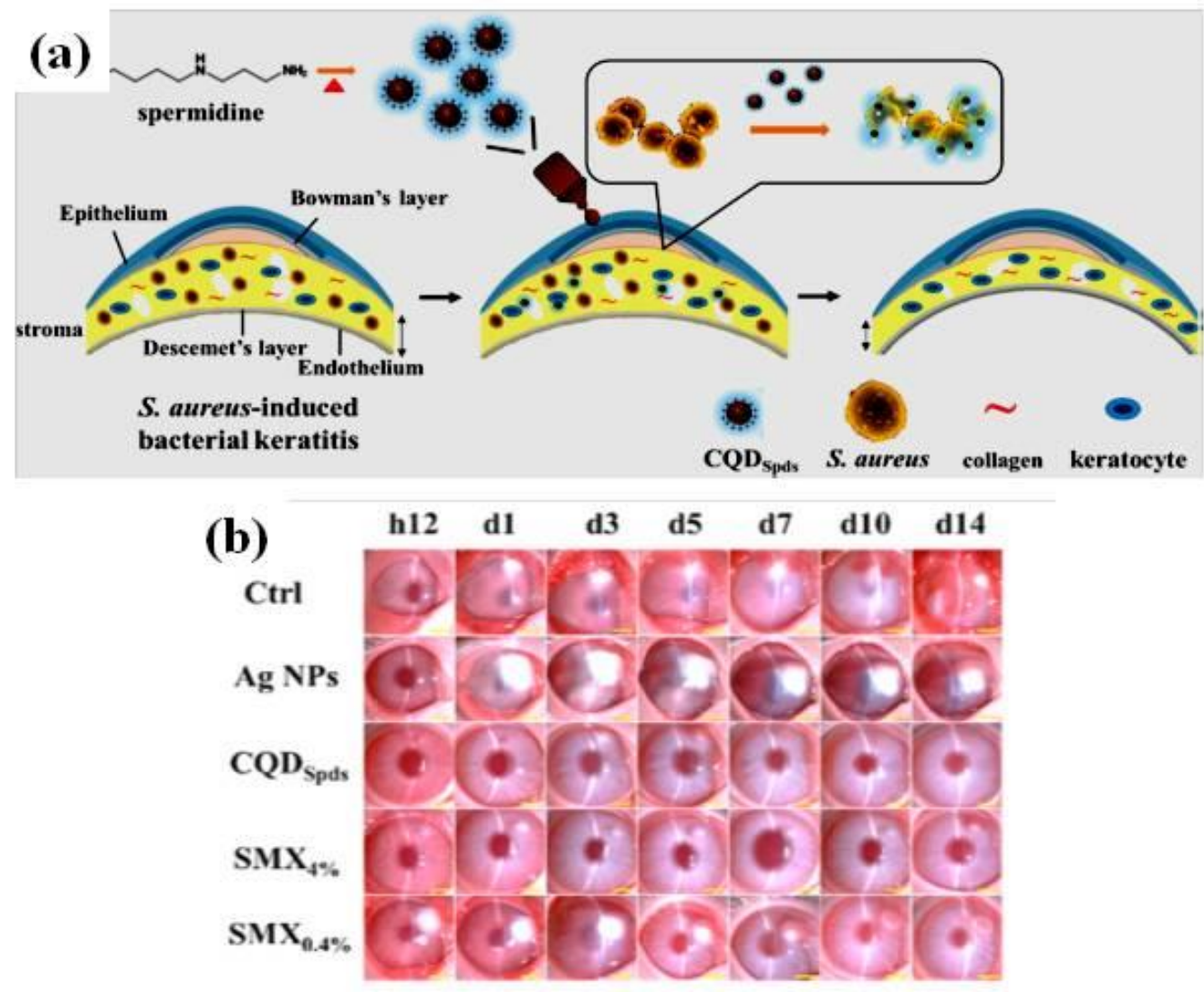

(B)

\section{(a)}

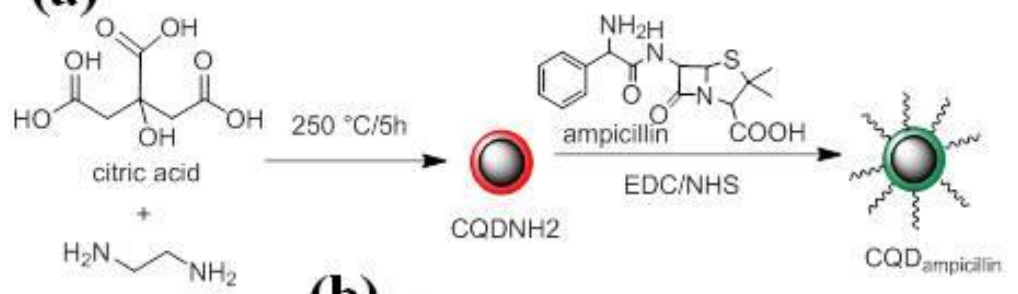

ethylenediamine

(b)
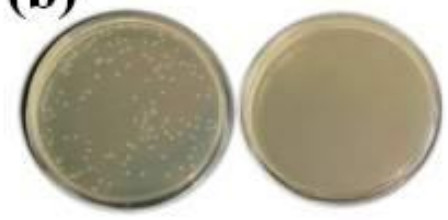

(c)

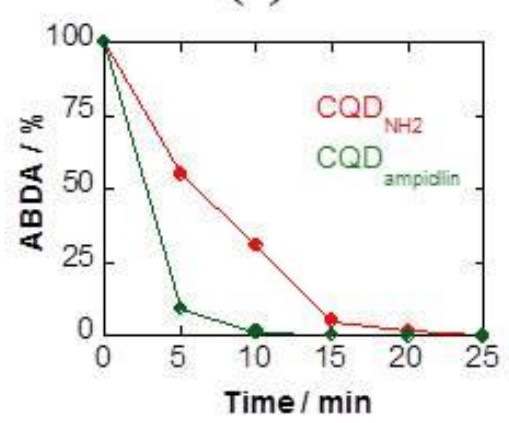

No light Visible light

Figure 16: Antibacterial applications: (A) (a) Use of spermidine derived fluorescent CQDs for the treatment of $S$. aureus induced bacterial keratitis, (b) Slit-lamp biomicroscopic images

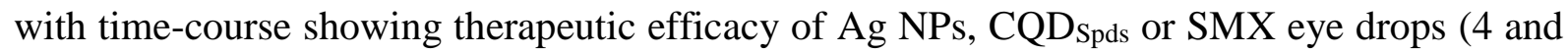
$0.4 \%$ ) after topical installation in rabbit eyes with experimentally induced bacterial keratitis (BK). S. aureus infects rabbis receiving PBS without nanomaterials or eye drops serve as a 
control (Ctrl) group (reprint with permission from Ref. ${ }^{230}$ ); (B) Use of ampicillin modified CQD (a) for ablation of E.coli cells: (b) microtiter plates of E.coli cells $\left(5 \times 10^{8} \mathrm{cfu} \mathrm{mL}^{-1}\right)$ when incubated with $\mathrm{CQD}_{\text {ampicillin }}\left(100 \mu \mathrm{g} \mathrm{mL}^{-1}\right)$ without and with visible light illumination (10 min, $1 \mathrm{~W} \mathrm{~cm}^{-2}$ ), (c) Decay curve of 9,10-anthracenediylbis(methylene)dimalonic acid (ABDA) absorption at $260 \mathrm{~nm}$ as a function of irradiation time in the presence of $\mathrm{CQD}_{\mathrm{NH} 2}$ and $\mathrm{CQD}_{\text {ampicillin }}\left(50 \mu \mathrm{g} \mathrm{mL}^{-1}\right)$ at $1 \mathrm{~W} \mathrm{~cm}^{-2} 237$.

CQDs are able to efficiently interact with viruses and block viral infectivity. ${ }^{40,} 239$ The potential of CQDs functionalized with boronic acid and/or amine functions to interfere with the entry of herpes simplex virus type 1 (HSV-1) was demonstrated by by some of us lately. ${ }^{40}$ CQDs formed from 4-aminophenylboronic acid hydrochloride (CQDs $\mathrm{AB}$ ), using a modified hydrothermal carbonization process, prevent HSV-1 infection in the $\mathrm{ng} / \mathrm{mL}$ concentration range $\left(\mathrm{EC}_{50}=80 \mathrm{ng} \mathrm{mL}^{-1}\right.$ and $145 \mathrm{ng} \mathrm{mL}^{-1}$ on Vero and $\mathrm{A} 549$ cells, respectively), whereas the corresponding CQDs formed from phenylboronic acid (CQDsB) had no effect even at high concentrations (Figure 17). Such CQDs have laely shown to considered a therapeutic options for the highly pathogenic human coronavirus (HCoV) infections. ${ }^{239}$ Optimized CQDs resulted in the development of the first-generation anti-HCoV nanomaterial with an effective inhibition concentration to achieve $50 \%$ inhibition $\left(\mathrm{EC}_{50}\right)$ of $11 \pm 5 \mu \mathrm{g} \mathrm{mL}^{-1}$. The underlying mechanism of action of these CQDs revealed to be due to inhibition of HCoV-229E entry that could be due to interaction of the boronic-acid functions of CQDs with HCoV-229E S protein via pseudolectin based interactions; surprisingly, an equally large inhibition activity was observed at the viral replication step. These results are extremely encouraging to replace currently used antiviral agents such a ribavirin and IFN known to have major side effects such as confusion, short-term memory loss, deficits in executive functions, as well as extrapyramidal effects. The approach has to be further tested in the clinical relevant MERS$\mathrm{CoV}$, to validate the potential of these nanostructures as alternative anti-MERS therapeutics and approaches to confront this severe and life threatening disease.
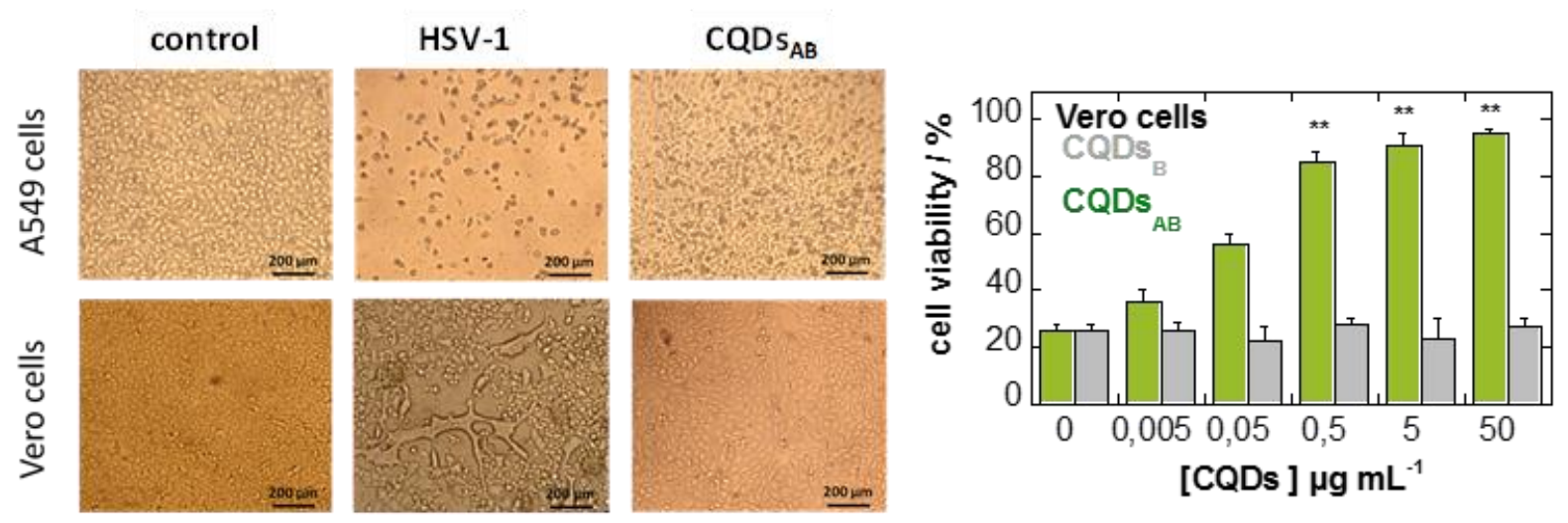

Figure 17: Antiviral treatment: Phenylboronic acid $\left(\mathrm{CQD}_{\mathrm{B}}\right)$ and 4-aminophenylboronic acid $\left(\mathrm{CQD}_{\mathrm{BA}}\right)$ derived particles for interfering with HSV-1 infection of A549 and Vero cell layers: (left) Inverted microscope iamge $(\times 10)$ after $24 \mathrm{~h}$ inoculation with HSV-1 or HSV-1 mixed 
with $\mathrm{CQD}_{\mathrm{B}}$ or $\mathrm{CQD}_{\mathrm{BA}}$ together with (right) percentage of Vero cells viability using Uptiblue ${ }^{\circledR}$ assay (reprint with permission form Ref. ${ }^{40}$ ).

\subsubsection{Inhibition of protein aggregation}

Motivated by the ability of NPs to cross the blood-brain-barrier, much effort has been devoted to understand the effect of nanoparticles on protein aggregation in the context of neurodegenerative diseases such as that of amyloid-beta peptide in Alzheimer's disease. CQDs $^{240-243}$ and GQDs ${ }^{244,}{ }^{245}$ positively impacted this field as they showed to be effective inhibitors. CQDs of $6 \mathrm{~nm}$ in size, prepared from carbon powder by acid treatment at elevated temperature, were able to efficiently inhibit insulin fibrillation in a concentration-dependent manner. The inhibition effect was observed at $0.2 \mu \mathrm{g} \mathrm{mL}^{-1}$, with $40 \mu \mathrm{g} \mathrm{mL}^{-1} \mathrm{CQDs}$ preventing $0.2 \mathrm{mg} \mathrm{mL} \mathrm{m}^{-1}$ of human insulin from fibrillation for 5 days under $65^{\circ} \mathrm{C}$, whereas insulin is denatured in $3 \mathrm{~h}$ under the same conditions in absence of CQDs. ${ }^{240}$ The key process to the success of CQDs inhibitors is that they are delivered into the brain. Using human transferrin modified CQDs and zebrafish as in vivo model, the ability of CQDs to cross the brain blood boundary (BBB) was evidenced. ${ }^{243}$

\section{Summary and Outlook}

In conclusion, we hope that focusing on CQDs and GQDs and bridging optical and electrochemical detection aspects of these materials, the interest of carbon-based quantum particles and their place among other carbon nanomaterials, such fullerenes, carbon nanotubes and graphene has been paved. While all started more than 15 years with the discovery of the one of the most attractive features of CQDs and GQDs, their tunable optical properties, these defect-rich chemical structures have since then revealed their potential to revolutionize optical and electroanalytical analysis and have shown to be a major player for biomedical related applications related to drug delivery, ablation of pathogens, interfering with viral attachment to cells and anti-fibrillation. One of the reasons responsible for the interest in CQDs and GQDs is that their synthesis is generally simple and their integration with other nanomaterials easy. Thus underlines the importance of the synthetic part of these particles. Even though a wide variety of procedures and a large number of starting materials have been investigated for their preparation, none of the nanostructures have made it yet into a marketable product. The challenge is here to improve and develop size and function controlled synthetic approaches. Questions about standardization and comparison have to be addressed to be able to compare the properties of each prepared nanomaterial. A substantial batch-to-batch and lab-to-lab variation exists with important consequences on the performance of carbon-based sensors as well their biocompatibility. As the fluorescence properties and the quantum yield are related to the overall composition and the presence of residual chemical groups on the surface of the CQDs and GQDs, standardization is highly required.

Linked to controlled synthetic approaches are the question of up scaling and the use of ecofriendly and low cost procedures. The use of green synthetic approaches, relying on starting materials such as orange juice, soya milk, black tea, pig skin, cabbage, rose petals, waste 
paper, agriculture waste and others is highly appealing and need to be considered for scale up synthesis. Most work reports the production of CQDs and GQDs at the gram scale, something which needs to be expanded in the future. Production of $120 \mathrm{~g}$ of CQDs has been reported via the carbonization of Chinese ink. However the energy input for carbonization is considerable, counter-balancing the green approach put into the forefront. The need for synthetic approaches at lower temperatures using such "green" starting materials remains still a challenge for materials scientists and chemists alike.

Photoluminescence remains one of the most attractive properties of CQDS and GQDs being however highly depending on the raw material used for CQDs production and the surface functions present on the surface of the particles. Fluorescence in CQDs produced from carbon is believed to $b$ due to radioactive recombination of surface-confined electrons and holes; in the case of CQDs from biomass and organic molecules, surface effects are put into the forefront. There is an uncertainty about chemical groups on the surface in most fabrication processes. Post-functionalization might help to overcome this issue, but these approaches are rarely employed as post-functionalization often decreases considerably the fluorescence quantum yield. This brings us to an important challenge: how to improve the quantum yield of CQDs allowing taking full advantage of their intrinsic properties and how to tune their emission spectrum. Nearly all CQDs show maximal fluorescence emission in the blue or green spectrum. As the optical properties are the base of highly sensitive sensors, altering of the band gap of these nanomaterials and consequently their spectral position and emission pathways by chemical modification through the addition of heteroatoms (doping) and chemical surface passivation is an active research. The development of CQDs with strong fluorescence emission in the NIR has in parallel become primordial for biomedical imaging to cross cells and tissue without harming it. Despite extensive studies, a complete fundamental understanding of the structure-property relationship remains unclear mainly due to the nonuniform nature of the particles in size, the heterogeneous chemical composition, and complex mechanistic considerations due to the quantized character of the particles.

The development of CQDs and GQDs with high quantum yield (QY) is a key requirement for most applications. Often QY are as low as $0.5-10 \%$, while QY of $80 \%$ are obtained with organic fluorescent dye such as Rhodamine 6G. Doping of CQDs with nitrogen and $\mathrm{Mg}$ ion is one of the mean for improving the QY. Compared to metal ion doping, heteroatoms show better advantages due to their atomic size comparable with carbon quantum dots. Questions about toxicity still have to be evaluated to see if these CQDs are appropriate for biomedical applications. How to engineer well-defined absorption band with high QY in the visible region remains a critical challenge.

The electrical conductivity of CQDs still remains little investigate and limits their use for electrical and electrochemical read outs. More research needs to be performed to underline the usability of CQDs as electrode material or as amplification strategy in electrochemical analysis. While the good water disperability makes these nanostructures well adapted for nanomedical applications, their solubility makes using classical electrode modification approach such as drop casting, spraying of limited use. In the case of electrochemical properties, different considerations might have to be taken into account such as charge, and the presence of electrochemical actives surface groups. It is believed that the numerous open 
questions concerning large-scale synthesis, product purity, quantum yield enhancement, visible to NIR emission will inspire researchers to contribute further to this field. The future of carbon-based quantum particles is rich and only a multidisciplinary approach taking advantage of experts in materials science, synthesis, surface chemistry, physics, analytical chemistry, bioimaging, etc. will result in success.

\section{REFERENCE}

1. X. Xu, R. Ray, Y. Gu, H. J. Ploehn, K. Gearheart, K. Raker and W. A. Scrivens, Electrophoretic analysis and purification of fluorescent single-walled carbon nanotube fragments. J. Am. Chem. Soc., 2004, 126, 12736-12737.

2. T. S. Atabaev, Doped Carbon Dots for Sensing and Bioimaging. Nanomat., 2018, 8, 342.

3. J. Zhang and S.-H. Yu, Carbon dots: large-scale synthesis, sensing and bioimaging. Mat. Today, 2016, 19, 382-393.

4. S. Y. Lim, W. Shen and Z. Gao, Carbon quantum dots and their applications. Chem. Soc. Rev. , 2015, 44, 362.

5. X. Qun and T. Lei, Fluorescent carbon dots and their sensing applications. Trends Anal. Chem., 2017, 89, 163-180.

6. V. Sharma, P. Tiwari and S. M. Mobin, Sustainable carbon-dots: recent advances in green carbon dots for sensing and bioimaging. J. Mater. Chem. B, 2017, 5, 8904-8892.

7. S. Zhu, Y. Song, X. Zhao, J. Shao, J. Zhang and Yang, The photoluminescence mechanism in carbon dots (graphene quantum dots, carbon nanodots, and polymer dots): current state and future perspectives. Nano Res., 2015, 8, 355-381.

8. Y. Du and S. Guo, Chemically doped fluorescent carbon and graphene quantum dots for bioimaging, sensor, catalytic and photoelectronic applications. Nanoscale, 2016, 8 , 2532-2543

9. Y. Park, J. Yoo, B. Lim, W. Kwon and S.-W. Rhee, Improving the fucntinality of carbon nanodies: dping and surface functianlization. J. Mater. Chem. A, 2016, 4, 11582.

10. R. Wang, K.-Q. Lu, Z.-R. Tang and T.-J. Xu, Recent progress in carbon quantum dots: synthesis, properties and applications in photocatalysis. J. Mater. Chem. A, 2017, 5, 3171 .

11. J. Zhang and S.-H. Yu, Carbon dots: large-scale synthesis, sensing and bioimaging. Materials Today, 2016, 19, 382-393.

12. B. De and N. Karak, Recent progress in carbon dot-metal based nanohybrids for photochemical and electrochemical applications J. Mater. Chem A, 2017, 5, 1826.

13. D. Iannazzo, I. Zicarelli and A. Pistone, Graphene quantum dots: multifunctinal nanoplatforms for anticancer therapy. J. Mater. Chem. B, 2017, 5, 6471.

14. M. L. Liu, B. B. Chen, C. M. Li and C. Z. Huang, Carbon dots: synthesis, formation mechanism, fluorescence origin and sensing applications. Green Chem., 2019, 21, 449-471

15. L. Xiao and H. Sun, Novel Properties and Applications of Carbon nano-dots. Nanoscale Horizon, 2018, 3, 565-597.

16. Y. Yulong and P. Xisheng, Recent advances in carbon-based dots for electroanalysis. Analyst, 2016, 141, 2619-2628. 
17. V. N. Mochalin, O. Shenderova, D. Ho and Y. Gogotsi, The Properties and Applications of Nanodiamonds. Nat. Nanotechnol., 2012, 7, 11-23.

18. K. Lawrence, C. L. Baker, T. D. James, S. D. Bull, R. Lawrence, J. M. Mitchels, M. Opallo, O. A. Arotiba, K. I. Ozoemena and F. Marken, Functionalized Carbon Nanoparticles, Blacks and Soots as Electron-Transfer Building Blocks and Conduits. Chem. Asian J., 2014, 9, 1226-1241.

19. K. Szot and M. Opallo, (Bio)electroanalytical Applications of Carbon nanoparticles. Electroanalysis, 2016, 28, 46-57.

20. A. I. Medalia and F. A. Heckman, Morphology of aggregates-II. Size and shape factores of carbon black aggregats from electron microscopy. Carbon, 1969, 7, 567582.

21. A. B. Siddique, A. K. Pramanick, S. Chatterjee and M. Ray, Amorphous Carbon Dots and their Remarkable Ability to Detect 2,4,6-Trinitrophenol. Sci. Rep., 2018, 8, 9770.

22. H. Li, Y. Liang, G. Yin, X. Wei and Z. Xu, Template-free fabrication of fullerene (C60, C70) nanomater-sized hollow spehres under solvothermal conditions. Carbon, 2008, 46, 1736-1740.

23. Y. Miyauchi, H. M. Hirori, K. and Y. Kanemitsu, Radiative lifetimes and coherence lengths of one-dimensional excitons in single-walled carbon nanotubes. Phys. Rev. B, 2009, 80, 081410.

24. C. Yang, C. Wu, X. Luo, X. Liu, Y. Gao, P. Wu, C. Cai and S. S. Saavedra, Exploring the Emissive States of Heteroatom-Doped Graphene Quantum Dots. J. Phys. Chem. C, 2018, 122, 6483-6492.

25. S. H. Jin, D. H. Kim, G. H. Jun, S. H. Hong and S. Jeon, Tuning the Photoluminescence of Graphene Quantum Dots through the Charge Transfer Effect of Functional Groups. ACS Nano, 2013, 7 1239-1245

26. S. Zhu, J. Zhang, S. Tang, C. Qiao, L. Wang, H. Wang, X. Liu, B. Li, Y. Li, W. Yu, $X$. Wang, H. Sun and B. Yang, Surface chemistry routes to modulate the photoluminescence of graphene quantum dots: from fluorescence mechanism to upconversion bioimaging applications. . Adv Func Mater., 2012, 22, 4732-4740.

27. S. Kim, S. W. Hwang, M.-H. Kim, D. Y. Shin, D. H. Shin, C. O. Kim, S. B. Yang, J. H. Park, E. Hwang, S.-H. Choi, G. Ko, S. Sim, C. Sone, H. J. Choi, S. Bae and B. H. Hong, Anomalous behaviors of visible luminescence from graphene quantum dots: interplay between size and shape. ACS Nano 2012, 6, 8203-8208.

28. Q. L. Zhao, Z. L. Zhang, B. H. Bi-Hai Huang, J. Peng, M. Zhang and D.-W. Pang, Facile preparation of low cytotoxicity fluorescent carbon nanocrystals by electrooxidation of graphite. Chem. Commun., 2008, 5116.

29. S. Ahirwar, S. Mallick and D. Bahadur, Electrochemical Method to prepare graphene quantum dots and graphene oxide quantum dots. ACS Omega, 2017, 2, 8343.

30. X. T. Zheng, A. Ananthanarayanan, K. Q. Luo and P. Chen, Glowing graphene quantum dots and carbon dots: properties, syntheses, and biological applications. Small, 2015, 11, 1620-1636.

31. J. Shen, Y. Zhu, X. Yang and C. G. Li, Graphene quantum dots: Emergent nanolights for bioimaging, sensors, catalysis and photovoltaic devices . Chem. Commun., 2012, 48, 3686.

32. W. Chen, G. Lv, W. Hu, D. Li, S. Chen and Z. Dai, Synthesis and applications of graphene quantum dots: a review. Nanotechnol. Rev., 2018, 7, 157-185.

33. H. Liu, T. Ye and C. Mao, Fluorescent carbon nanoparticles derived from candle soot. Angew. Chem. Int. Ed., 2007, 46, 6473-6475. 
34. D. Reyes, M. Camacho, M. Camacho, M. Mayorga, D. Weathers, G. Salamo, Z. Wang and A. Neogi, Laser Ablation carbon nanodots for light emission. Nanoscale Res. Lett., 2016, 11, 424.

35. C. Hu, Y. Liu, Y. Yang, J. Cui, Z. Huang, Y. Wang, L. Yang, H. Wang, Y. Xiao and J. Rong, One-step preparation of nitrogen-doped graphene quantum dots from oxidized debris of graphene oxide. J. Mater. Chem. B, 2013, 1, 39.

36. G. S. Kumar, R. Roy, D. Sen, U. K. Ghorai, R. Thapa, N. Mazumder, S. Sahab and K. K. Chattopadhyay, Amino-functionalized graphene quantum dots: origin of tunable heterogeneous photoluminescence. Nanoscale, 2013, 6, 3384.

37. D. B. Shinde and V. K. Pillai, Electrochemical preparaiton of luminescent grpahen quantum dots from multiwalled carbon nanotubes. Chem. Eur. J., 2012, 18, 1252212528 .

38. C.-I. Wang, W.-C. Wu, A. P. Periasamya and H.-T. Chang, Electrochemical synthesis of photoluminescent carbon nanodots from glycine for highly sensitive detection of hemoglobin. Green Chem., 2014, 16, 2509-2514.

39. S. Dey, A. Govindaraj, K. Biswas and C. N. R. Rao, Luminescence properties of boron and nitrogen doped graphene quantum dots prepared from arc-dischargegenerated doped graphene samples. Chem. Phys. Lett., 2014, 595-596, 203-208.

40. A. Barras, Q. Pagneux, F. Sane, Q. Wang, R. Boukherroub, D. Hober and S. Szunerits, High Efficiency of Functional Carbon Nanodots as Entry Inhibitors of Herpes Simplex Virus Type 1. ACS Appl. Mater. Interfaces, 2016, 8, 9004-9013.

41. L. Tang, R. Ji, X. Cao, J. Lin, H. Jiang, X. Li, K. S. Teng, X. M. Luk, S. Zeng, J. Hao and S. P. Lau, Deep Ultraviolet Photoluminescence of Water-Soluble Self-Passivated Graphene Quantum Dots. ACS Nano, 2012, 6, 5102-5110.

42. J. Wang, C. F. Wan and S. Chen, Amphiphilic egg-derived carbon dots: rapid plasma fabrication, pyrolysis process, and multicolor printing patterns. Angew. Chem. Int. Ed., 2012, 51, 9297-9301.

43. N. Arora and N. N. Sharma, Arc discharge synthesis of carbon nanotubes: comprehensive review. Diam. Rel. Mater., 2014, 50, 135-150.

44. G. K. Yogesh, E. P. Shuaib and D. Sastikumar, Photoluminescence Properties of Carbon Nanoparticles Synthesized from Activated Carbon Powder (4\% ash) by Laser Ablation in Solution. Mater. Res. Bull., 2017, 91, 220-226.

45. N. Tarasenka, A. Stupak, N. Tarasenko, S. Chakrabarti and D. Mariotti, Structure and Optical Properties of Carbon Nanoparticles Generated by Laser Treatment of Graphite in Liquids. ChemPhysChem, 2017, 18, 1074-1083.

46. J. Zhou, C. Booker, R. Li, X. Zhou, T.-K. Sham, X. Sun and Z. Ding, An electrochemical avenue to blue luminescent nanocrystals from multiwalled carbon nanotubes (MWCNTs). J. Am. Chem. Soc., 2007, 129, 744-745.

47. H. Li, X. He, Z. Kang, H. Huang, Y. Liu, J. Liu, S. Lian, C. H. Tsang, X. Yang and S. T. Lee, Water-soluble fluorescent carbon quantum dots and photocatalyst design. Angew. Chem. Int. Ed., 2010, 49, 4430-4434.

48. H. P. Liu, T. Ye and C. D. Mao, Fluorescent carbon nanoparticles derived from candle soot. Angew. Chem. Int. Ed., 2007, 46, 6473.

49. Y. Dong, N. Zhou, X. Lin, J. Lin, Y. Chi and G. Chen, Extraction of electrochemiluminescent oxidized carbon quantum dots from activated carbon. Chem. Mater., 2010, 22, 5895-5899.

50. K. M. Tripathi, A. Tyagi, M. Ashfaqa and R. G. Gupta, Temperature dependent, shape variant synthesis of photoluminescent and biocompatible carbon nanostructures from almond husk for applications in dye removal. RSC Adv., 2016, 6, 29545-29553 
51. Y. Yang, J. Cui, M. Zheng, C. Hu, S. Tan, Y. Xiao, Q. Yang and Y. Liu, One-step synthesis of amino-functionalized fluorescent carbon nanoparticles by hydrothermal carbonization of chitosan. Chem. Commun., 2012, 48, 380-382.

52. Z.-C. Yang, M. Wang, A. M. Yong, S. Y. Wong, Zhang, X.-H., H. Tan, A. Y. Chang, $\mathrm{X}$. Li and J. Wang, Intrinsically fluorescent carbon dots with tunable emission derived from hydrothermal treatment of glucose in the presence of monopotassium phosphate. Chem. Commun., 2011, 47, 11615.

53. P. C. Che, Y.-N. Chen, P.-C. Hsu, C.-C. Shih and H.-T. Chang, Photoluminescent organosilane-functionalized carbon dots as temperature probes. Chem. Commun., 2013, 49, 1639-1641.

54. H. K. Sadhanala, J. Khatei and K. K. Nanda, Facile hydrothermal synthesis of carbon nanoparticles and possible application as white light phosphors and catalysts for the reduction of nitrophenol. $R S C A d v ., 2014,4,11481-11485$.

55. J.-Y. Li, Y. Liu, Q.-W. Shu, J.-M. Liang, F. Zhang, X.-P. Chen, X.-P. Deng, M. T. Swihart and K.-J. Tan, One-Pot Hydrothermal Synthesis of Carbon Dots with Efficient Up- and Down-Converted Photoluminescence for the Sensitive Detection of Morin in a Dual-Readout Assay. Langmuir, 2017, 33, 1043.

56. S. K. Bhunia, A. Saha, A. R. Maity, S. C. Ray and N. R. Jana, Carbon Nanoparticlebased Fluorescent Bioimaging Probes. Sci. Rep., 2013, 3, 1473.

57. R. L. Liu, D. Q. Wu, S. H. Liu, K. Koynov, W. Knoll and Q. Li, An aqueous route to multicolor photoluminescent carbon dots using silica spheres as carriers. Angew. Chem. Int. Ed., 2009, 121, 4668-4671.

58. Y. Liu, N. Xiao, N. Gong, H. Wang, X. Shi, W. Gu and L. Ye, One-step microwaveassisted polyol synthesis of green luminescent carbon dots as optical nanoprobes. Carbon, 2014, 68, 258-264.

59. X. Zhai, C. Zhang, T. Bai, W. Li, L. Dai and W. Liu, Highly luminescent carbon nanodots by microwave-assisted pyrolysis. Chem. Commun., 2012, 48, 7955.

60. Y. Zhao, S. Zuo and M. Miao, The effect of oxygen on the microwave-assisted synthesis of carbon quantum dots from polyethylenbe glycik. RSC Adv., 2017, 7, 16637-16643.

61. S. Yang, X. Chen, S. Liu, F. Wang and G. Ouyang, Microwave-assisted solid-phase synthesis of highly fluorescent carbon nanoparticles and its application in intracellular pH sensing. Talanta, 2018, 186, 80-87.

62. H. Zhu, X. Wang, Y. Li, Z. Wang, F. Yang and X. Yang, Microwave synthesis of fluorescent crbon nanoparticles with electrochemiluinescent properties. Chem. Commun., 2009, 5118.

63. S. Yang, J. Sun, X. Li, W. Zhou, Z. Wang, P. Pen He, G. Ding, X. Xie, Z. Kang and M. Jianga, Large-scale fabrication of heavy doped carbon quantum dots with tunablephotoluminescence and sensitive fluorescence detection. J. Mater. Chem. A, 2014, 2, 8660 .

64. J. Hou, J. Yan, Q. Zhao, Y. Li, H. Ding and L. Ding, A novel one-pot route for largescale preparation of highly photoluminescent carbon quantum dots powders. Nanoscale, 2013, 5, 9558-9561

65. B. B. Chen, Z. X. Liu, W. C. Deng, L. Zhan, M. L. Liua and C. Z. Huang, A largescale synthesis of photoluminescent carbon quantum dots: a self-exothermic reaction driving the formation of the nanocrystalline core at room temperature. Green Chem., 2016, 18, 5127-5132 
66. R. Yan, H. Wu, Q. Zheng, J. Wang, J. Huang, K. Ding, Q. Guo and J. Wang, Graphene quantum dots cut from graphene flakes: High electrocatalyticactivity for oxygen reduction and low cytotoxicity. RSC Adv., 2014, 4, 23097.

67. H. Liu, Q. Wang, G. Shen, C. Zhang, C. Li, W. Ji, C. Wang and D. Cui, A multifunctional ribonuclease A-conjugated carbon dot cluster nanosystem for synchronous cancer imaging and therapy. Nanoscale Res. Lett., 2014, 9, 397.

68. P. Roy, P.-C. Chen, A. P. Periasamy, Y.-N. Chen and H.-T. Chang, Photoluminescent carbon nanodots: synthesis, physicochemical properties and analytical applications. Mater. Today, 2015, 18, 447-458.

69. G. Eda, Y. Y. Lin, C. Mattevi, H. Yamaguchi, H. A. Chen, I. S. Chen, C. W. Chen and M. Chhowalla, Blue photoluminescence from chemically derived graphene oxide. Adv. Mater., 2010, 22, 505-509.

70. L. Cao, X. Wang, M. J. Meziani, F. Lu, H. Wang, P. G. Luo, Y. Lin, B. A. Harruff, L. M. Veca, D. Murray, S.-Y. Xie and Y.-P. Sun, Carbon Dots for Multiphoton Bioimaging. J. Am. Chem. Soc., 2007, 129, 11318-11319.

71. Y. Dong, C. Chen, J. Lin, N. Zhou, Y. Chi and G. Chen, Electrochemiluminescence emission from carbon quantumdot-sulfite coreactant system. Carbon, 2013, 56, 12-17.

72. Y. Xu, J. Liu, C. Gao and E. Wang, Applications of carbon quantum dots in electrochemiluminescence: A mini review. Electrochem. Commun., 2014, 48, 151154.

73. N. Li, L. P. Jia, R. N. Ma, W. L. Jia, Y. Y. Lu, S. S. Shi and H. S. Wang, A novel sandwiched electrochemiluminescence immunosensor for the detection of carcinoembryonic antigen based on carbon quantum dots and signal amplification. Biosens. Bioelectron., 2017, 89, 453-460.

74. Y.-P. Sun, B. Zhou, Y. Lin, W. Wang, K. A. Shiral Fernando, P. Pathak, M. J. Meziani, B. A. Harruff, X. Wang, H. Wang, P. G. Luo, H. Yang, H. E. Kose, B. Chen, L. M. Veca and S.-Y. Xie, Quantum-Sized Carbon Dots for Bright and Colorful Photoluminescence. J. Am. Chem. Soc., 2006, 128, 7756-7757.

75. A. S. R. Bati, L. P. Yu, M. Batmunkh and J. G. Shapter, Synthesis, purification, properties and characterization of sorted single-walled carbon nanotubes. Nanoscale, 2018, 10, 22087-22139.

76. C. S. Lim, K. Hola, A. Ambrosi, R. Zboril and M. Pumera, Graphene and carbon quantum dots electrochemistry. Electrochem. Commun., 2015, 52, 75-79.

77. F. J. Xia, M. Pan, S. C. Mu, M. D. Jones, G. Kociok-Kohn, S. C. Tsang and F. Marken, Imparting $\mathrm{pH}$ - and small molecule selectivity to nano-Pd catalysts via hydrothermal wrapping with chitosan. Electrochim. Acta, 2013, 110, 663-669.

78. F. J. Xia, M. Pan, S. C. Mu, M. D. Jones, D. Wolverson and F. Marken, ChitosanBased Hydrothermal Nanocarbon: Core-Shell Characteristics and Composite Electrodes. Electroanalysis, 24, 1703-1708.

79. F. J. Xia, M. Pan, S. C. Mu, Y. L. Xiong, K. J. Edler, I. Idini, M. D. Jones, S. C. Tsang and F. Marken, Hydrothermal core-shell carbon nanoparticle films: thinning the shell leads to dramatic $\mathrm{pH}$ response. Phys. Chem. Chem. Phys., 14, 15860-15865.

80. J. Xi, C. Xie, Y. Zhang, L. Wang, J. Xiao, X. Duan, J. Ren, F. Xiao and S. Wang, Pd Nanoparticles Decorated N-Doped Graphene Quantum Dots@N-Doped Carbon Hollow Nanospheres with High Electrochemical Sensing Performance in Cancer Detection. ACS Appl. Mater. Interfaces, 2016, 8, 22563-22573.

81. X. Duan, J. Xu, Z. Wei, J. Ma, S. Guo, S. Wang, H. Liu and S. Dou, Metal-free carbon materials for CO2 electrochemical reduction. Adv. Mater., 2017, 29, 1701784. 
82. P.-C. Hsu, Z.-Y. Shih, C.-H. Lee and H.-T. Chang, Synthesis and analytical applications of photoluminescent carbon nanodots Green Chem., 2012, 14, 917-920

83. J. Yan, S. eHou, Y. Yu, Y. Qiao, T. Xiao, Y. Mei, Z. Zhang, B. Wang, C. C. Huang, C. H. Lin and G. Suo, The effect of surface charge on the cytotoxicity and uptake of carbon quantum dots in human umbilical cord derived mesenchymal stem cells. Colloids Sur. B: Biointerfaces, 20187, 171, 241-249.

84. Y.-F. Kang, Y.-H. Li, Y.-W. Fang, Y. Xu, X.-M. Wei and X.-B. Yin, Carbon quantum dots for zebrafish fluorescence imaging. Sci. Rep. , 2015, 5, 11835.

85. M. Havrdova, K. Hola, J. Skopalik, K. Tomankova, M. Petr, K. Cepe, K. Polakova, J. Tucek, A. B. Bourlinos and R. Zboril, Toxicity of carbon dots - Effect of surface functionalization on the cell viability, reactive oxygen species generation and cell cycle. Carbon, 2016, 99, 238-248.

86. S.-T. Yang, L. Cao, P. G. Luo, F. Lu, X. Wang, H. Wang, M. J. Meziani, Y. Liu, G. Qi and Y.-P. Sun, Carbon Dots for Optical Imaging in Vivo. J. Am. Chem. Soc., 2009, 131, 11308-11309.

87. S. Li, Z. Guo, Y. Zhang, W. Xue and Z. Liu, Blood Compatibility Evaluations of Fluorescent Carbon Dots. ACS Appl Mater Interfaces. , 2015, 7, 19153-19162.

88. D. Zhong, Y. Zhuo, Y. Feng and X. Yang, Employing carbon dots modified with vancomycin for assaying Gram-positive bacteria like Staphylococcus aureus. Biosens. Bioelectron., 2015, 74, 546-553.

89. J. Chen, Y. Li, K. Lv, W. Zhong, H. Wang, Z. Wu, P. Yi and J. Jiang, Cyclamfunctionalized carbon dots sensor for sensitive and selective detection of copper (II) ion and sulfide anion in aqueous media and its imaging in live cells. Sens. Actuator B, 2016, 224, 298-306.

90. Y. Kim, G. Jang and T. S. Lee, New fluorescent metal-ion detection using a paperbased sensor strip containing tethered rhodamine carbon nanodots. ACS applied materials \& interfaces, 2015, 7, 15649-15657.

91. E. Ju, Z. Liu, Y. Du, Y. Tao, J. Ren and X. Qu, Heterogeneous assembled nanocomplexes for ratiometric detection of highly reactive oxygen species in vitro and in vivo. ACS Nano, 2014, 8, 6014-6023.

92. H. Valizadeh, A. Shomali, S. Nourshargh and M. Mohammad-Rezaei, Carboxyl and nitrite functionalized graphene quantum dots as a highly active reagent and catalyst for rapid diazotization reaction and synthesis of azo-dyes under solvent-free conditions. Dyes and Pigments, 2015, 113, 522-528.

93. A. A. Mohamed, Z. Salmi, S. A. Dahoumane, A. Mekki and M. M. Chehimi, Functionalization of nanomaterials with aryldiazonium salts. Adv. Colloid Interface Sci. , 2015, 225, 16-36.

94. H. Jin, R. Gui and J. Sun, Carrot-derived carbon dots modified with polyethyleneimine and nile blue for ratiometric two-photon fluorescence turn-on sensing of sulfide anion in biological fluids. Talanta, 2017, 169, 141.

95. F. R. Baptista, S. A. Belhout, S. Giordani and S. J. Quinn, Recent developments in carbon nanomaterials sensors. Chem. Soc. Rev., 2015, 44, 4433-4453.

96. Y. Li, Y. Zhong, Y. Zhang, W. Weng and S. Li, Carbon quantum dots/octahedral $\mathrm{Cu} 2 \mathrm{O}$ nanocomposites for non-enzymatic glucose and hydrogen peroxide amperometric sensor. Sens. Actuator B, 2015, 206, 735.

97. S. Chowdhury, B. Rooj, A. Dutta and U. Mandal, Review on Recent Advances in Metal Ions Sensing Using Different Fluorescent Probes. J. Fluoresc., 2018, 28, 9991021. 
98. W. Lu, X. Qin, S. Liu, G. Chang, Y. Zhang, Y. Luo, A. M. Asiri, A. O. Al-Youbi and X. Sun, Economical, Green Synthesis of Fluorescent Carbon Nanoparticles and Their Use as Probes for Sensitive and Selective Detection of Mercury(II) Ions. Anal. Chem., 2012, 84, 5351.

99. H. M. Goncalves, A. J. Duarte and J. C. Esteves da Silva, Optical fiber sensor for $\mathrm{Hg}(\mathrm{II})$ based on carbon dots. Biosens. Bioelectron., 2010, 26, 1302.

100. S. Barman and M. Sadhukhan, Facile bulk production of highly blue fluorescent graphitic carbon nitride quantum dots and their application as highly selective and sensitive sensors for the detection of mercuric and iodide ions in aqueous media $J$. Mater. Chem., 2012, 22, 21832.

101. H. Lu, S. Qi, J. Mack, Z. Li, J. Lei, N. Kobayashi and Z. Shen, Facile $\mathrm{Hg}^{2+}$ detection in water using fluorescent self-assembled monolayers of a rhodamine-based turn-on chemodosimeter formed via a "click" reaction J. Mater. Chem., 2011, 21, 10878.

102. P.-p. Zhu, Z. Cheng, L.-1. Du, Q. Chen and K.-j. Tan, Synthesis of the Cu-Doped Dual-Emission Fluorescent Carbon Dots and Its Analytical Application. Langmuir, 2018, 34, 9982-9989.

103. C. Tan, X. Su, C. Zhou, B. Wang, Q. Zhan and S. He, Acid-assisted hydrothermal synthesis of red fluorescent carbon dots for sensitive detection of Fe(iii). RSC Adv., 2017, 7, 40952-40956.

104. X.-C. Fu, J.-Z. Jin, J. Wu, J.-C. Jin and C.-G. Xie, A novel turn-on fluorescent sensor for highly selective detection of Al(iii) in an aqueous solution based on simple electrochemically synthesized carbon dots. Anal. Meth., 2017, 9, 3941-3948.

105. D. A. Chowdhury and R.-a. Doong, Highly Sensitive and Selective Detection of Nanomolar Ferric Ions Using Dopamine Functionalized Graphene Quantum Dots. ACS Appl. Mater. Interfaces, 2016, 8, 21002-21010.

106. V. Sharma, A. K. Saini and S. M. Mobin, Multicolour fluorescent carbon nanoparticle probes for live cell imaging and dual palladium and mercury sensors. J. Mater. Chem. $B, 2016,4,2466-2476$.

107. J. Feng, Y. Chen, Y. Han, J. Liu, C. Ren and X. Chen, Fluorescent carbon nanoparticles: A low-temperature trypsin-assisted preparation and $\mathrm{Fe}^{3+}$ sensing. Anal. Chim. Acta, 2016, 926, 107-117.

108. K. Srinivasan, K. Subramanian, K. Murugan and K. Dinakaran, Sensitive fluorescence detection of mercury(ii) in aqueous solution by the fluorescence quenching effect of $\mathrm{MoS}_{2}$ with DNA functionalized carbon dots. Analyst, 2016, 141, 6344-6352.

109. S. Liu, J. Tian, L. Wang, Y. Zhang, X. Qin, Y. Luo, A. M. Asiri, A. O. Al-Youbi and $X$. Sun, Hydrothermal treatment of grass: a low-cost, green route to nitrogen-doped, carbon-rich, photoluminescent polymer nanodots as an effective fluorescent sensing platform for label-free detection of $\mathrm{Cu}(\mathrm{II})$ ions. Adv. Mater., 2012, 24, 2037.

110. F. Y. Yan, Sens. Actuator B-Chem., 2014, 192, 488.

111. F. Yan, Y. Zou, M. Wang, X. Mu, N. Yang and L. Chen, Highly photoluminescent carbon dots-based fluorescent chemosensors for sensitive and selective detection of mercury ions and application of imaging in living cells. Sens. Actuator B, 2014, 192, 488-495.

112. R. M. Duke, E. B. Veale, F. M. Pfeffer, P. R. Kruger and T. Gunnlaugsson, Colorimetric and fluorescent anion sensors: an overview of recent developments in the use of 1,8-naphthalimide-based chemosensors. Chem. Soc. Rev., 2010, 39, 3936-3953.

113. C. Yu, X. Li, F. Zeng, F. Zhend and S. Wu, Carbon-dot-based ratiometric fluorescent sensor for detecting hydrogen sulfide in aqueous media and inside live cells Chem. Commun., 2013, 49, 403. 
114. S. Sun, K. Jiang, S. Qian, Y. Wang and H. Lin, Applying Carbon Dots-Metal Ions Ensembles as a Multichannel Fluorescent Sensor Array: Detection and Discrimination of Phosphate Anions. Anal. Chem., 2017, 89, 5542-5548.

115. Y. Hu, J. Yang, J. Tian and J.-S. Yu, Waste frying oil as a precursor for one-step synthesis of sulfur-doped carbon dots with $\mathrm{pH}$-sensitive photoluminescence. Carbon, 2014, 77, 775 .

116. M. Zheng, X. Zhigang, D. Dan Qu, D. Di Li, P. Peng Du, X. Jing and Z. Sun, OnOff-On Fluorescent Carbon Dot Nanosensor for Recognition of Chromium(VI) and Ascorbic Acid Based on the Inner Filter Effect. ACS Appl. Mater. Interfaces, 2013, 5, 13242.

117. J. Shangguan, D. He, X. He, K. Wang, F. Xu, J. Liu, J. Tang, X. Yang and J. Huang, Label-Free Carbon-Dots-Based Ratiometric Fluorescence $\mathrm{pH}$ Nanoprobes for Intracellular pH Sensing. Anal. Chem., 2016, 88, 7837-7843.

118. Y. Wang, L. Lu, H. Peng, J. Xu, F. Wang, R. Qi, Z. Xu and W. Zhang, Multi-doped carbon dots with ratiometric $\mathrm{pH}$ sensing properties for monitoring enzyme catalytic reactions. Chem. Commun, 2016, 52, 9247-9250.

119. Y. Feng, D. Zhong, H. Miao and K. Yang, Carbon dots derived from rose flowers for tetracycline sensing. Talanta, 2015, 140, 128.

120. B. Campos, T. J. Bandosz and J. Jimenez-Jimenez, Carbon Dots as Fluorescent Sensor for Detection of Explosive Nitrocompounds. Carbon, 2016, 106, 171.

121. S. Devi, R. K. Gupta, A. K. Paul and S. Tyagi, Waste carbon paper derivatized Carbon Quantum Dots/(3-Aminopropyl)triethoxysilane based fluorescent probe for trinitrotoluene detection. Mater. Res. Exp., 2019, 6, 025605.

122. S. Mandani, B. Sharma, D. Dey and T. K. Sarma, Carbon nanodots as ligand exchange probes in Au@C-dot nanobeacons for fluorescent turn-on detection of biothiols. Nanoscale, 2015, 7, 1802-1808.

123. X. Zhou, A. Wang, C. Yu, S. Wu and J. Shen, Facile Synthesis of Molecularly Imprinted Graphene Quantum Dots for the Determination of Dopamine with AffinityAdjustable. ACS Applied Materials \& Interfaces, 2015, 7, 11741-11747.

124. E. Zor, E. Morales-Narváez, A. Zamora-Gálvez, H. Bingol, M. Ersoz and A. Merkoçi, Graphene Quantum Dots-based Photoluminescent Sensor: A Multifunctional Composite for Pesticide Detection. ACS Appl. Mater. Interfaces, 2015, 7, 2027220279.

125. K. M. Tripathi, A. K. Sonker, A. Bhati, J. Bhuyan, A. Singh, A. Singh, S. Sarkar and S. K. Sonkar, Large-scale synthesis of soluble graphitic hollow carbon nanorods with tunable photoluminescence for the selective fluorescent detection of DNA. New J. Chem., 2016, 40, 1571-1579.

126. A. H. Loo, Z. Sofer, D. Bouša, P. Ulbrich, A. Bonanni and M. Pumera, Carboxylic carbon quantum dots as a fluorescent sensing platform for DNA detection. ACS Appl. Mater. Interfaces, 2016, 8, 1951-1957.

127. N. Wang, W. Yiting, G. Tingting, Y. Ting, C. Mingli and W. ianhua, Green preparation of carbon dots with papaya as carbon source for effective fluorescent sensing of Iron(III) and Escherichia coli. Biosens. Bioelectron., 2016, 85, 68-75.

128. L. Zheng, Y. Chi, Y. Dong, J. Lin and B. Wang, Electrochemiluminescence of WaterSoluble Carbon Nanocrystals Released Electrochemically from Graphite. J. Am. Chem. Soc., 2009, 131, 4564-4565.

129. L. Li, D. Liu, H. Mao and T. You, Multifunctional solid-state electrochemiluminescence sensing platform based on poly(ethylenimine) capped Ndoped carbon dots as novel co-reactant. Biosens. Bioelectron., 2017, 89, 489-495. 
130. S. Zhu, X. Lin, P. Ran, F. Mo, Q. Xia and Y. Fu, A glassy carbon electrode modified with C-dots and silver nanoparticles for enzymatic electrochemiluminescent detection of glutamate enantiomers. Microchim. Acta, 2017, 184, 4679-4684.

131. Q. Liu, C. Ma, X. P. Liu, Y. P. Wei, C. J. Mao and J. J. Zhu, A novel electrochemiluminescence biosensor for the detection of microRNAs based on a DNA functionalized nitrogen doped carbon quantum dots as signal enhancers. Biosens. Bioelectron., 2017, 92, 273-279.

132. W.-J. Niu, R.-H. Zhu, S. Cosnier, X.-J. Zhang and D. Shan, Ferrocyanide-Ferricyanide Redox Couple Induced Electrochemiluminescence Amplification of Carbon Dots for Ultrasensitive Sensing of Glutathione. Anal. Chem., 2015, 87, 11150-11156.

133. J. Lu, Yan M, L. Ge, S. Ge, S. Wang, J. Yan and J. Yu, Electrochemiluminescence of blue-luminescent graphene quantum dots and its application in ultrasensitive aptasensor for adenosine triphosphate detection. Biosens. Bioelectron., 2013, 47, 271.

134. J. Shangguan, J. Huang, D. He, X. He, K. Wang, R. Ye, X. Yang, T. Qing and J. Tang, Highly Fe3+-selective fluorescent nanoprobe based on ultrabright N/P codoped carbon dots and its application in biological samples. Anal. Chem., 2017, 89, 7477-7484.

135. V. Sharma, N. Kaur, P. Tiwari and S. M. Mobin, Multifunctional fluorescent "Off-OnOff" nanosensor for $\mathrm{Au}^{3+}$ and $\mathrm{S}^{2-}$ employing $\mathrm{N}-\mathrm{S}$ co-doped carbon-dots. Carbon, 2018, 139, 393-403.

136. A. Tyagi, K. M. Tripathi, N. Singh, S. Choudhary and R. K. Gupta, Green synthesis of carbon quantum dots from lemon peel waste: applications in sensing and photocatalysis. RSC Adv., 2016, 6, 72423-72432.

137. X. Zhu, Z. Zhang, Z. Xue, C. Huang, Y. Shan, C. Liu, X. Qin, W. Yang, X. Chen and T. Wang, Understanding the Selective Detection of Fe3+ Based on Graphene Quantum Dots as Fluorescent Probes: The Ksp of a Metal Hydroxide-Assisted Mechanism. Anal. Chem., 2017, 89, 12054-12058.

138. M. Yang, Q. Tang, Y. Meng, J. Liu, T. Feng, X. Zhao, S. Zhu, W. Yu and B. Yang, Reversible "Off-On" Fluorescence of Zn2+-Passivated Carbon Dots: Mechanism and Potential for the Detection of EDTA and $\mathrm{Zn}^{2+}$. Langmuir, 2018, 34, 7767-7775.

139. L. Wang, M. Li, W. Li, Y. Han, Y. Liu, Z. Li, B. Zhang and D. Pan, Rationally Designed an Efficient Dual-Mode Colorimetric/Fluorescence Sensor Based on Carbon Dots for Detection of pH and Cu2+ Ions. ACS Sustain. Chem. Eng., 2018.

140. V. Sharma, N. Kaur, P. Tiwari and S. M. Mobin, Full color emitting fluorescent carbon material as reversible $\mathrm{pH}$ sensor with multicolor live cell imaging. $J$. Photochem. Photobiol. B, 2018, 182, 137-145.

141. W. Liu, C. Li, X. Sun, W. Pan and J. Wang, Carbon-dot-based ratiometric fluorescent $\mathrm{pH}$ sensor for the detections of very weak acids assisted by auxiliary reagents that contribute to the release of protons. Sens. Actuator B, 2017, 244, 441-449.

142. B. B. Chen, M. L. Liu, L. Zhan, C. M. Li and C. Z. Huang, Terbium(III) Modified Fluorescent Carbon Dots for Highly Selective and Sensitive Ratiometry of Stringent. Analytical Chemistry, 2018, 90, 4003-4009.

143. T.-Y. Wang, C.-Y. Chen, C.-M. Wang, Y. Z. Tan and W.-S. Liao, Multicolor functional carbon dots via one-step refluxing synthesis. ACS sensors, 2017, 2, 354363.

144. Y. Wu, X. Liu, Q. Wu, J. Yi and G. Zhang, Carbon Nanodots-Based Fluorescent TurnOn Sensor Array for Biothiols. Anal. Chem., 2017, 89, 7084.

145. H. Li, W. Kong, J. Liu, N. Liu, H. Huang, Y. Liu and Z. Kang, Fluorescent N-doped carbon dots for both cellular imaging and highly-sensitive catechol detection. Carbon, 2015, 91, 66. 
146. H. Li, J. Liu, S. Guo, Y. Zhang, H. Huang, Y. Liu and Z. Kang, Carbon dots from PEG for highly sensitive detection of levodopa J. Mater Chem. B, 2015, 3, 2378.

147. K. M. Tripathi, A. K. Sonker, A. Bhati, J. Bhuyan, A. Singh, A. Singh, S. Sarkar and S. K. Sonkar, Large-scale synthesis of soluble graphitic hollow carbon nanorods with tunable photoluminescence for the selective fluorescent detection of DNA. New. J. Chem., 2016, 40, 1571.

148. R. Jalili and M. Amjadi, Surface molecular imprinting on silane-functionalized carbon dots for selective recognition of nifedipine. RSC Adv., 2015, 5, 74084-74090.

149. S. Devi, R. K. Gupta, A. K. Paul, V. Kumar, A. Sachdev, P. Gopinath and S. Tyagi, Ethylenediamine mediated luminescence enhancement of pollutant derivatized carbon quantum dots for intracellular trinitrotoluene detecction: soot to shine. RSC $A d v$., 2018, 8, 32684-32694.

150. R. K. Mishra, I. N. Pulidindi, E. Kabha and A. Gedanken, In situ formation of carbon dots aids ampicillin sensing. Anal. Meth., 2016, 8, 2441-2447.

151. H. Xu, X. Yang, G. Li, C. Zhao and X. Liao, Green Synthesis of Fluorescent Carbon Dots for Selective Detection of Tartrazine in Food Samples. J. Agric. Food Chem., 2015, 63, 6707-6714.

152. S. Li, J. Li, J. Luo, Q. Zhang and L. Zhang, A fluorescence switch sensor for detection of virginiamycin based on graphene oxide-supported carbon quantum dots and molecularly imprinted polymer. $R S C A d v$., 2017, 7, 56359-56364.

153. Y. He, X. Wang, J. Sun, S. Jiao, H. Chen, F. Gao and L. Wang, Fluorescent blood glucose monitor by hemin-functionalized graphene quantum dots based sensing system. Anal. Chim. Acta, 2014, 810, 71-78.

154. Z.-B. Qu, X. Zhou, L. Gu, R. Lan, D. Sun, D. Yu and G. Shi, Boronic acid functionalized graphene quantum dots as a fluorescent probe for selective and sensitive glucose determination in microdialysate Chem. Commun., 2013, 49, 9830.

155. L. Zhang, Z.-Y. Zhang, R.-P. Liang, Y.-H. Li and J.-D. Qiu, Boron-Doped Graphene Quantum Dots for Selective Glucose Sensing Based on the "Abnormal" AggregationInduced Photoluminescence Enhancement. Anal. Chem., 2014, 86, 4423.

156. L. Li, G. Wu, T. Hong, Z. Yin, D. Sun, E. S. Abdel-Halim and J.-J. Zhu, Graphene Quantum Dots as Fluorescence Probes for Turn-off Sensing of Melamine in the Presence of Hg2+. ACS Applied Materials \& Interfaces, 2014, 6, 2858-2864.

157. S. Benítez-Martínez, Á. I. López-Lorente and M. Valcárcel, Graphene Quantum Dots Sensor for the Determination of Graphene Oxide in Environmental Water Samples. Anal. Chem., 2014, 86, 12279-12284.

158. H.-J. Li, X. Sun, F. Xue, N. Ou, B.-W. Sun, D.-J. Qian, M. Chen, D. Wang, J. Yang and X. Wang, Redox Induced Fluorescence On-Off Switching Based on Nitrogen Enriched Graphene Quantum Dots for Formaldehyde Detection and Bioimaging. ACS Sustainable Chemistry \& Engineering, 2018, 6, 1708-1716.

159. J. Wang, Modified electrodes for electrochemical sensors. Electroanalysis, 1991, 3, 255-259.

160. L. Vidal, A. Chisvert, A. Canals, E. Psillakis, A. Lapkin, F. Acosta, K. J. Edler, J. A. Holdaway and F. Marken, Chemically surface-modified carbon nanoparticle carrier for phenolic pollutants: Extraction and electrochemical determination of benzophenone-3 and triclosan. Anal. Chim. Acta, 2008, 616, 28.

161. H. Salehniya, M. Amiri and F. Marken, Voltammetric Chloride Sensing Based on Trace-Level Mercury Impregnation Into Amine-Functionalized Carbon Nanoparticle Films. IEEE Sensors Journal, 2017, 17, 5437-5443. 
162. M. Amiri, S. Ghaffari, A. Bezaatpour and F. Marken, Carbon nanoparticle-chitosan composite electrode with anion, cation, and neutral binding sites: Dihydroxybenzene selectivity. Sens. Actuator B, 2012, 162, 194-200.

163. A. Celebanska, D. Tomaszewska, A. Lesniewski and M. Opallo, Film electrode prepared from oppositely charged silicate submicroparticles and carbon nanoparticles for selective dopamine sensing. Biosens. Bioelectron., 2011, 26, 4417.

164. M. Amiri, F. Rezapour and A. Bezaatpour, Hydrophilic carbon nanoparticulates at the surface of carbon paste electrode improve determination of paracetamol, phenylephrine and dextromethorphan. J. Electroanal. Chem., 2014, 735, 10-18.

165. M. Amiri, E. Ghasempour and A. Bezaatpour, Alizarin-modified sulfonate carbon nanoparticles in vanadium sensing. J. Solid State Electrochem., 2014, 18, 1005-1013.

166. H. Salehniya, M. Amiri and Y. Mansoori, Positively charged carbon nanoparticulate/sodium dodecyl sulphate bilayer electrode for extraction and voltammetric determination of ciprofloxacin in real samples. RSC Adv., 2016, 6, 30867-30874.

167. J. Pishahang, H. B. Amiri and H. Heli, Synthesis of carbon nanoparticles-poly (orthoaminophenol) nanocomposite and its application for electroanalysis of iodate. Sens. Actuator B, 2018, 256, 878-887.

168. M. Amiri, H. Eynaki and Y. Mansoori, Cysteine-anchored receptor on carbon nanoparticles for dopamine sensing. Electrochim. Acta, 2014, 123, 362-368.

169. D. B. Shinde and V. K. Pillai, Electrochemical Resolution of Multiple Redox Events for Graphene Quantum Dots. Angew. Chem. Int. Ed., 2013, 52, 2482-2485.

170. L. J. Wang, G. Cao, T. Tu, H. O. Li, X. J. Zhou, Z. Hao, G. Su, C. Guo, H. W. Jiang and G. P. Guo, A graphene quantum dot with a single electron transistor as an integrated charge sensor App. Phys. Lett., 2010, 97, 262113.

171. L. Li, D. Liu, A. Shi and T. You, Simultaneous stripping determination of cadmium and lead ions based on the $\mathrm{N}$-doped carbon quantum dots-graphene oxide hybrid. Sens. Actuator B, 2018, 255, 1762-1770.

172. M. Shou, C. R. Ferrario, K. N. Schultz, T. E. Robinson and R. T. Kennedy, Monitoring dopamine in vivo by microdialysis sampling and on-line CE-laser-induced fluorescence. Anal. Chem., 2006, 78, 6717-6725.

173. M. Algarra, A. González-Calabuig, K. Radotić, D. Mutavdzic, C. O. Ania, J. M. Lázaro-Martínez, J. Jiménez-Jiménez, E. Rodríguez-Castellón and M. del Valle, Enhanced electrochemical response of carbon quantum dot modified electrodes. Talanta, 2018, 178, 679-685.

174. J. Chen, P. He, H. Bai, S. He, T. Zhang, X. Zhang and F. Dong, Poly $(\beta-$ cyclodextrin)/carbon quantum dots modified glassy carbon electrode: Preparation, characterization and simultaneous electrochemical determination of dopamine, uric acid and tryptophan. Sensors and Actuators B: Chemical, 2017, 252, 9-16.

175. Y. Jiang, B. Wang, F. Meng, Y. Cheng and C. Zhu, Microwave-assisted preparation of $\mathrm{N}$-doped carbon dots as a biosensor for electrochemical dopamine detection. Journal of colloid and interface science, 2015, 452, 199-202.

176. H. Wang, R. Li and Z. Li, Nanohybrid of $\mathrm{Co} 3 \mathrm{O} 4$ and histidine-functionalized graphene quantum dots for electrochemical detection of hydroquinone. Electrochimica Acta, 2017, 255, 323-334.

177. F. Tan, L. Cong, X. Li, Q. Zhao, H. Zhao, X. Quan and J. Chen, An electrochemical sensor based on molecularly imprinted polypyrrole/graphene quantum dots composite for detection of bisphenol A in water samples. Sensors and Actuators B: Chemical, 2016, 233, 599-606. 
178. B. B. Prasad, A. Kumar and R. Singh, Synthesis of novel monomeric graphene quantum dots and corresponding nanocomposite with molecularly imprinted polymer for electrochemical detection of an anticancerous ifosfamide drug. Biosensors and Bioelectronics, 2017, 94, 1-9.

179. Z. Zhao, Q. Ou, X. Xiuwen Yin and J. Jianqi Liu, Nanomaterial-based electrochemical hydrogen peroxide biosenso. Int. J. Biosen. Bioelectron. , 2017, 7, 100-102.

180. G. Muthusankar, R. Sasikumar, S.-M. Chen, G. Gopu, N. Sengottuvelan and S.-P. Rwei, Electrochemical synthesis of nitrogen-doped carbon quantum dots decorated copper oxide for the sensitive and selective detection of non-steroidal antiinflammatory drug in berries. Journal of colloid and interface science, 2018, 523, 191200.

181. H. Razmi and R. Mohammad-Rezaei, Graphene quantum dots as a new substrate for immobilization and direct electrochemistry of glucose oxidase: application to sensitive glucose determination. Biosens. Bioelectron., 2013, 41, 498-504.

182. Y. Su, X. Zhou, Y. Long and W. Li, Immobilization of horseradish peroxidase on amino-functionalized carbon dots for the sensitive detection of hydrogen peroxide. Microchim. Acta, 2018, 185, 114.

183. Y. Zhang, C. Y. Wu, X. J. Zhou, X. C. Wu, Y. Q. Yang, H. X. Wu, S. W. Guo and J. Y. Zhang, Graphene quantum dots/gold electrode and its application in living cell $\mathrm{H} 2 \mathrm{O} 2$ detection. Nanoscale, 2013.

184. M. L. Yola and N. Atar, A novel detection approach for serotonin by graphene quantum dots/two-dimensional (2D) hexagonal boron nitride nanosheets with molecularly imprinted polymer. Applied Surface Science, 2018, 458, 648-655.

185. H. Guo, H. Jin, R. Gui, Z. Wang, J. Xia and F. Zhang, Electrodeposition one-step preparation of silver nanoparticles/carbon dots/reduced graphene oxide ternary dendritic nanocomposites for sensitive detection of doxorubicin. Sensors and Actuators B: Chemical, 2017, 253, 50-57.

186. Z. Cai, F. Li, P. Wu, L. Ji, H. Zhang, C. Cai and D. F. Gervasio, Synthesis of nitrogendoped graphene quantum dots at low temperature for electrochemical sensing trinitrotoluene. Anal. Chem., 2015, 87, 11803-11811.

187. M. Arvand and S. Hemmati, Magnetic nanoparticles embedded with graphene quantum dots and multiwalled carbon nanotubes as a sensing platform for electrochemical detection of progesterone. Sensors and Actuators B: Chemical, 2017, 238, 346-356.

188. M. Arvand and S. Hemmati, Analytical methodology for the electro-catalytic determination of estradiol and progesterone based on graphene quantum dots and poly (sulfosalicylic acid) co-modified electrode. Talanta, 2017, 174, 243-255.

189. A. Mars, B. Bouhaouala-Zahar and N. Raouafi, Ultrasensitive sensing of Androctonus australis hector scorpion venom toxins in biological fluids using an electrochemical graphene quantum dots/nanobody-based platform. Talanta, 2018, 190, 182-187.

190. T. C. Canevari, F. H. Cincotto, M. Nakamura, S. A. S. Machado and H. E. Toma, Efficient electrochemical biosensors for ethynylestradiol based on the laccase enzyme supported on single walled carbon nanotubes decorated with nanocrystalline carbon quantum dots. Anal. Meth., 2016, 8, 7254-7259.

191. L. Li, D. Liu, A. Shi and T. You, Simultaneous stripping determination of cadmium and lead ions based on the $\mathrm{N}$-doped carbon quantum dots-graphene oxide hybrid. Sensors and Actuators B: Chemical, 2018, 255, 1762-1770.

192. M. Saraf, K. Natarajan and S. M. Mobin, Robust Nanocomposite of Nitrogen-Doped Reduced Graphene Oxide and MnO2 Nanorods for High-Performance Supercapacitors 
and Nonenzymatic Peroxide Sensors. ACS Sustainable Chem. Eng., 2018, 6, 1048910504.

193. F. Chekin, F. Teodorescu, Y. Coffinier, G. H. Pan, A. Barras, R. Boukherroub and S. Szunerits, $\mathrm{MoS}_{2} /$ reduced graphene oxide as active hybrid material for the electrochemical detection of folic acid in human serum. Biosens. Bioelectron., 2016, 85, 807-813.

194. W. Zheng, H. Wu, Y. Jiang, J. Xu, X. Li, W. Zhang and F. Qiu, A molecularlyimprinted-electrochemical-sensor modified with nano-carbon-dots with high sensitivity and selectivity for rapid determination of glucose. Anal. Biochem., 2018, 555, 42-49.

195. M. Jahanbakhshi and B. Habibi, A novel and facile synthesis of carbon quantum dots via salep hydrothermal treatment as the silver nanoparticles support: Application to electroanalytical determination of $\mathrm{H} 2 \mathrm{O} 2$ in fetal bovine serum. Biosensors and Bioelectronics, 2016, 81, 143-150.

196. N. Amini, M. Shamsipur, M. B. Gholivand and A. Barati, A glassy carbon electrode modified with carbon quantum dots and polyalizarin yellow $\mathrm{R}$ dyes for enhanced electrocatalytic oxidation and nanomolar detection of L-cysteine. Microchemical Journal, 2017, 131, 9-14.

197. N. R. Devi, T. V. Kumar and A. K. Sundramoorthy, Electrochemically Exfoliated Carbon Quantum Dots Modified Electrodes for Detection of Dopamine Neurotransmitter. J. Electrochem. Soc., 2018, 165, G3112-G3119.

198. J. Fang, Z. Xie, G. Wallace and X. Wang, Co-deposition of carbon dots and reduced graphene oxide nanosheets on carbon-fiber microelectrode surface for selective detection of dopamine. Applied Surface Science, 2017, 412, 131-137.

199. L. Yu, X. Yue, R. Yang, S. Jing and L. Qu, A sensitive and low toxicity electrochemical sensor for 2, 4-dichlorophenol based on the nanocomposite of carbon dots, hexadecyltrimethyl ammonium bromide and chitosan. Sensors and Actuators B: Chemical, 2016, 224, 241-247.

200. H. Guo, R. Gui, H. Jin and Z. Wang, Facile construction of reduced graphene oxidecarbon dot complex embedded molecularly imprinted polymers for dual-amplification and selective electrochemical sensing of rutoside. New J. Chem., 2017, 41, 9977-9983.

201. S. Shiri, N. Pajouheshpoor, H. Khoshsafar, S. Amidi and H. Bagheri, An electrochemical sensor for the simultaneous determination of rifampicin and isoniazid using a C-dots@CuFe2O4 nanocomposite modified carbon paste electrode. New J. Chem., 2017, 41, 15564-15573.

202. T. Sarkar, H. Bohidar and P. R. Solanki, Carbon dots-modified chitosan based electrochemical biosensing platform for detection of vitamin D. Int. J. Biol. Macromol., 2018, 109, 687-697.

203. L. Wang, X. Chen, C. Liu and W. Yang, Non-enzymatic acetylcholine electrochemical biosensor based on flower-like NiAl layered double hydroxides decorated with carbon dots. Sens. Actuator B, 2016, 233, 199-205.

204. H. V. Nguyen, L. Richtera, A. Moulick, K. Xhaxhiu, J. Kudr, N. Cernei, H. Polanska, Z. Heger, M. Masarik and P. Kopel, Electrochemical sensing of etoposide using carbon quantum dot modified glassy carbon electrode. Analyst, 2016, 141, 2665-2675.

205. Q. Huang, X. Lin, J. J. Zhu and Q. X. Tong, Pd-Au@carbon dots nanocomposite: Facile synthesis and application as an ultrasensitive electrochemical biosensor for determination of colitoxin DNA in human serum. Biosens. Bioelectron., 2017, 94, 507-512. 
206. W. Guo, F. Pi, H. Zhang, J. Sun, Y. Zhang and X. Sun, A novel molecularly imprinted electrochemical sensor modified with carbon dots, chitosan, gold nanoparticles for the determination of patulin. Biosens. Bioelectron., 2017, 98, 299-304.

207. R. M. Shereema, T. P. Rao, V. B. Sameer Kumar, T. V. Sruthi, R. Vishnu, G. R. D. Prabhu and S. Sharath Shankar, Individual and simultaneous electrochemical determination of metanil yellow and curcumin on carbon quantum dots based glassy carbon electrode. Materials Science and Engineering: C, 2018, 93, 21-27.

208. J. Ju and W. Chen, In Situ Growth of Surfactant-Free Gold Nanoparticles on NitrogenDoped Graphene Quantum Dots for Electrochemical Detection of Hydrogen Peroxide in Biological Environments. Anal. Chem., 2015, 87, 1903-1910.

209. S. Samuei, J. Fakkar, Z. Rezvani, A. Shomali and B. Habibi, Synthesis and characterization of graphene quantum dots/CoNiAl-layered double-hydroxide nanocomposite: Application as a glucose sensor. Anal. Biochem., 2017, 521, 31-39.

210. A. Shamsi, F. Ahour and B. Sehatnia, Nickel oxide nanoparticles decorated graphene quantum dot as an effective electrode modifier for electrocatalytic oxidation and analysis of clozapine. Journal of Solid State Electrochemistry, 2018, 1-9.

211. E. Habibi and H. Heidari, Renewable Surface Carbon-composite Electrode Bulk Modified with GQD-RuCl3 Nano-composite for High Sensitive Detection of 1-tyrosine. Electroanalysis, 2016, 28, 2559-2564.

212. N. Shadjou, M. Hasanzadeh, F. Talebi and A. P. Marjani, Graphene quantum dot functionalized by beta-cyclodextrin: a novel nanocomposite toward amplification of 1cysteine electro-oxidation signals. Nanocomposites, 2016, 2, 18-28.

213. R. Shadjou, M. Hasanzadeh, M. Heidar-poor and N. Shadjou, Electrochemical monitoring of aflatoxin M1 in milk samples using silver nanoparticles dispersed on a-cyclodextrin-GQD s nanocomposite. J. Mol. Recog., 2018, 31, e2699.

214. N. Hashemzadeh, M. Hasanzadeh, N. Shadjou, J. Eivazi-Ziaei, M. Khoubnasabjafari and A. Jouyban, Graphene quantum dot modified glassy carbon electrode for the determination of doxorubicin hydrochloride in human plasma. Journal of Pharmaceutical Analysis, 2016, 6, 235-241.

215. X. Jian, X. Liu, H.-m. Yang, M.-m. Guo, X.-l. Song, H.-y. Dai and Z.-h. Liang, Graphene quantum dots modified glassy carbon electrode via electrostatic selfassembly strategy and its application. Electrochim. Acta, 2016, 190, 455-462.

216. S. Baluta, A. Lesiak and J. Cabaj, Graphene Quantum Dots-Based Electrochemical Biosensor for Catecholamine Neurotransmitters Detection. Electroanalysis, 2018, 30, 1781-1790.

217. J. Y. Huang, L. Zhao, W. Lei, W. Wen, Y. J. Wang, T. Bao, H. Y. Xiong, X. H. Zhang and S. F. Wang, A high-sensitivity electrochemical aptasensor of carcinoembryonic antigen based on graphene quantum dots-ionic liquid-nafion nanomatrix and DNAzyme-assisted signal amplification strategy. Biosensors and Bioelectronics, 2018, 99, 28-33.

218. B. Bali Prasad, A. Kumar and R. Singh, Synthesis of novel monomeric graphene quantum dots and corresponding nanocomposite with molecularly imprinted polymer for electrochemical detection of an anticancerous ifosfamide drug. Biosens. Bioelectron., 2017, 94, 1-9.

219. S. L. Ting, S. J. Ee, A. Ananthanarayanan, K. C. Leong and P. Chen, Graphene quantum dots functionalized gold nanoparticles for sensitive electrochemical detection of heavy metal ions. Electrochim. Acta, 2015, 172, 7-11. 
220. F. Ahour and M. Taheri, Anodic stripping voltammetric determination of copper (II) ions at a graphene quantum dot-modified pencil graphite electrode. Journal of the Iranian Chemical Society, 2018, 15, 343-350.

221. S. K. Guin, A. S. Ambolikar, J. Paul Guin and S. Neogy, Exploring the excellent photophysical and electrochemical properties of graphene quantum dots for complementary sensing of uranium. Sensors and Actuators B: Chemical, 2018, 272, 559-573.

222. M. Roushani and Z. Abdi, Novel electrochemical sensor based on graphene quantum dots/riboflavin nanocomposite for the detection of persulfate. Sens. Actuator B, 2014, 201, 503-510.

223. S. Behzadi, V. Serpooshan, W. Tao, M. A. Hamaly, M. Y. Alkawareek , E. C. Dreaden, D. Brown, A. M. Alkilany, O. C. Farokhzad and M. Mahmoudi, Cellular uptake of nanoparticles: journey inside the cell. Chem. Soc. Rev. , 2017, 17, 42184244. .

224. H. Q. Tao, K. Yang, Z. Ma, J. Wan, Y. Zhang, Z. Kang and Z. X. Liu, In vivo NIr fluorescence imaging, biodistribution and toxicology of photoluminescence carbon dots produced from carbon nanotubes and graphite. Small, 2012, 8, 281-290.

225. L. M. Pan, Q. He, J. Liu, Y. Chgen, M. Ma, L. Zhang and J. Shij, Nuclear-Targeted Drug Delivery of TAT Peptide-Conjugated Monodisperse Mesoporous Silica Nanoparticles. J. Am. Chem. Soc., 2012, 134, 5722-5725.

226. Z. X., Y. Zhang, C. Wang, X. C. Wu, Y. Yang, B. Zheng, H. X. Wu, S. Guo and J. Zhang, Photo-Fenton Reaction of Graphene Oxide: A New Strategy to Prepare Graphene Quantum Dots for DNA Cleavage. ACS Nano, 2012, 6, 6592-6599.

227. C. Wang, C. Y. Wu, X. J. Zhou, T. Han, X. Xin, J. Wu, J. Zhang and S. Guo, Enhancing cell nucleus accumulation and DNA cleavage activity of anti-cancer drug via graphene quantum dots. Sci. Rep., 2013, 3, 2852.

228. H. Chen, Z. Wang, S. Zong, P. Chen, D. Zhu, L. Wu and Y. Cui, A graphene quantum dot-based FRET system for nuclear-targeted and real-time monitoring of drug delivery Nanoscale, 2015, 7, 15477-15486.

229. W. Bing, H. Sun, Z. Yan, J. Ren and X. Qu, Programmed Bacteria Death Induced by Carbon Dots with Different Surface Charge. Small, 2016, 12, 4713-4718.

230. H.-J. Jian, R.-S. Wu, T.-Y. Lin, Y.-J. Li, H.-J. Lin, S. G. Harroun, J.-Y. Lai and C.-C. Huang, Super-Cationic Carbon Quantum Dots Synthesized from Spermidine as an Eye Drop Formulation for Topical Treatment of Bacterial Keratitis. ACS Nano, 2017, 11, 6703-6716.

231. S. G. Harroun, J.-Y. Lai, C.-C. Huang, S.-K. Tsai and H.-J. Lin, Reborn from the Ashes: Turning Organic Molecules to Antimicrobial Carbon Quantum Dots. ACS infectious diseases, 2017, 3, 777-779.

232. J. Yang, X. Zhang, Y.-H. Ma, G. Gao, X. Chen, H.-R. Jia, Y.-H. Li, Z. Chen and F.-G. We, Carbon Dot-Based Platform for Simultaneous Bacterial Distinguishment and Antibacterial Applications. ACS Appl Mater Interfaces., 2016, 8, 32170-32181.

233. L. Hiu, J. Huang, G. Chen, Y. Zhu and L. Yang, Antibacterial Property of Graphene Quantum Dots (Both Source Material and Bacterial Shape Matter). ACS Applied Materials \& Interfaces, 2016, 8, 20-25.

234. H. Sun, N. GAo, J. Dong, J. Ren and X. Qu, Graphene Quantum Dots-Band-Aids Used for Wound Disinfection. ACS Nano, 2014, 8, 6202-6210.

235. B. Z. Ristic, M. M. Milenkovic, I. R. Dakic, B. M. Todorovic-Markovic, M. S. Milosavljevic, M. D. Budimir, V. G. Paunovic, M. D. Dramicanin, Z. M. Markovic 
and V. S. Trajkovic, Photodynamic antibacterial effect of graphene quantum dots. Biomaterials, 2014, 35, 4428.

236. W.-S. Kuo, S. Yu-Ting, K.-S. Huang, T.-M. Chou and C.-H. Yang, Antimicrobial Amino-Functionalized Nitrogen-Doped Graphene Quantum Dots for Eliminating Multidrug-Resistant Species in Dual-Modality Photodynamic Therapy and Bioimaging under Two-Photon Excitation. ACS Appl. Mater. Interfaces, 2018, 10, 14438-14446.

237. R. Jijie, A. Barras, J. Bouckaert, N. Dumitrascu, S. Szunerits and R. Boukherroub, Enhanced antibacterial activity of carbon dots functionalized with ampicillin combined with visible light triggered photodynamic effects. Colloids Surf. B: Biointerfaces, 2018, 170, 347-354.

238. M. J. Meziani, X. Dong, L. Zhu, L. P. Jones, G. E. LeCroy, F. Yang, S. Wang, P. Wang, Y. Zhao, L. Yang, R. A. Tripp and Y.-P. Sun, Visible-Light-Activated Bactericidal Functions of Carbon "Quantum" Dots. ACS Applied Materials \& Interfaces, 2016, 8, 10761.

239. A. Łoczechin, K. Seron, A. Barras, N. Metzler-Nolte, R. Boukherroub, J. Dubuisson and S. Szunerits, Functional C-dots as medical countermeasures to Middle East Respiratory Syndrome (MERS) Coronavirus ACS Appl. Mater. Interfaces, 2018, under revision.

240. S. Li, L. Wang, C. C. Chusuei, V. M. Suarez, P. L. Blackwelder, M. Micic, J. Orbulesu and R. M. Leblanc, Nontoxic carbon dots potently inhibit human insulin fibrillation. Chem. Mater., 2015, 27, 1764-1771.

241. Q. Q. Yang, J. C. Jin, Z. Q. Xu, J. Q. Zhang, B. B. Wang, F. L. Jiang and Y. Liu, Active site-targeted carbon dots for the inhibition of human insulin fibrillation $J$. Mater. Chem. B, 2017, 5, 2010-2018.

242. M. Holubova, R. Konefal, A. Zhigunov, J. Svoboda, O. Pop-Georgievski, J. Hromadkova, O. Groborz, P. Stepanek and M. Hruby, Carbon nanospecies affecting amyloid formation $R S C A d v ., 2017,7,53887$.

243. X. Han, Z. Jing, W.-C. Wu, B. Zou, Z. Peng, P. Ren, A. Wikramanayake, Z. Lu and R. M. Leblanc, Biocompatible and blood-brain barrier permeable carbon dots for inhibition of Ab fibrillation and toxicity, and BACE1 activity. Nanoscale, 2017, 9, 12862.

244. M. Yousaf, H. Huang, P. Li, C. Wang and Y. Yang, Fluorine Functionalized Graphene Quantum Dots as Inhibitor against hIAPP Amyloid Aggregation. ACS Chem. Neurosci., 2017, 8, 1368.

245. H. J. Zeng, Miao M2, Z. Liu, R. Yang and L. B. Qu, Effect of nitrogen-doped graphene quantum dots on the fibrillation of hen egg-white lysozyme. Int. J. Biol. Macromol., 2017, 95, 856-861. 